Network Working Group

Request for Comments: 2670

Category: Proposed Standard
M. St. Johns, Ed. a Home Network August 1999

\title{
Radio Frequency ( $R F)$ Interface Management Information Base for MCNS/DOCSIS compliant RF interfaces
}

\section{Status of this Memo}

This document specifies an Internet standards track protocol for the Internet community, and requests discussion and suggestions for improvements. Please refer to the current edition of the "Internet Official Protocol Standards" (STD 1) for the standardization state and status of this protocol. Distribution of this memo is unlimited.

Copyright Notice

Copyright (C) The Internet Society (1999). All Rights Reserved.

Abstract

This memo defines a portion of the Management Information Base (MIB) for use with network management protocols in the Internet community. In particular, it defines a basic set of managed objects for SNMPbased management of MCNS/DOCSIS compliant Radio Frequency (RF) interfaces.

This memo specifies a MIB module in a manner that is compliant to the SNMP SMIV2 [5][6][7]. The set of objects are consistent with the SNMP framework and existing SNMP standards.

This memo is a product of the IPCDN working group within the Internet Engineering Task Force. Comments are solicited and should be addressed to the working group's mailing list at ipcdneterayon.com and/or the author.

Table of Contents

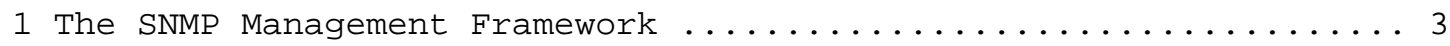

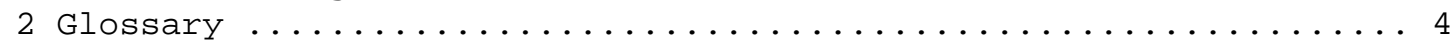

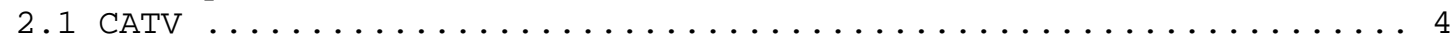

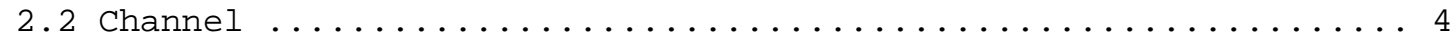

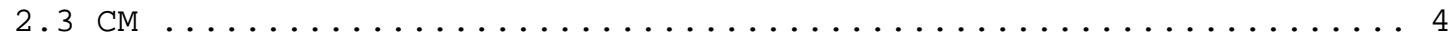

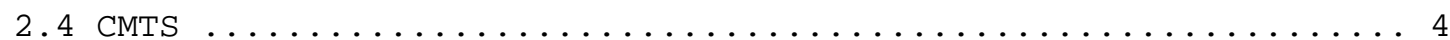

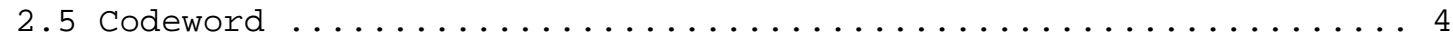

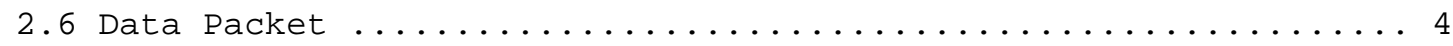




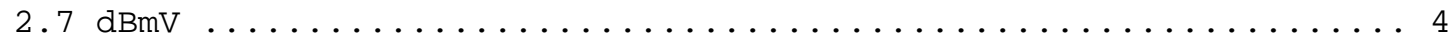

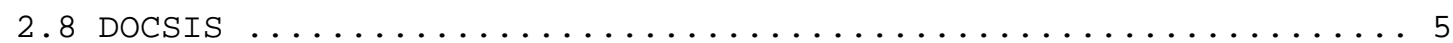

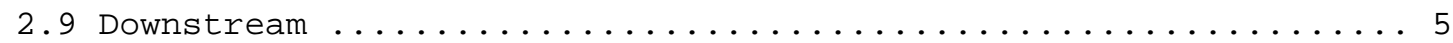

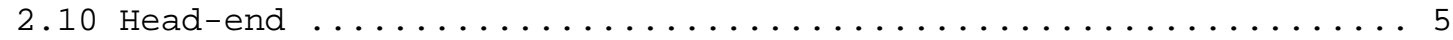

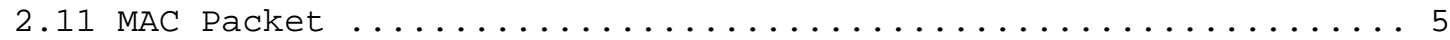

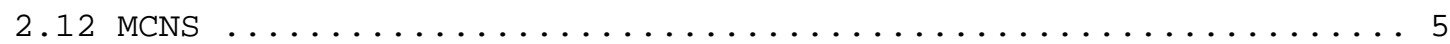

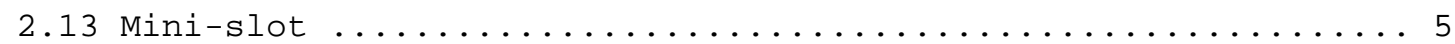

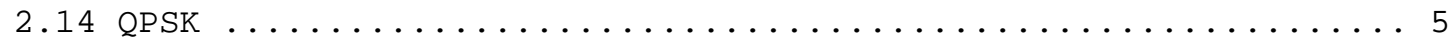

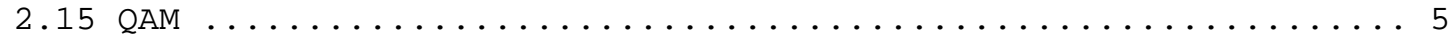

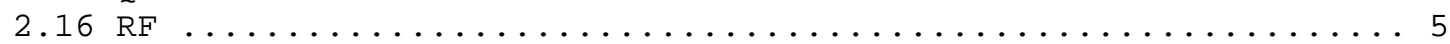

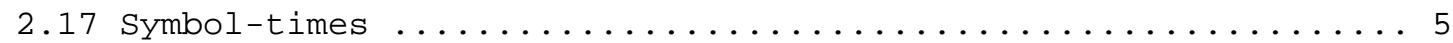

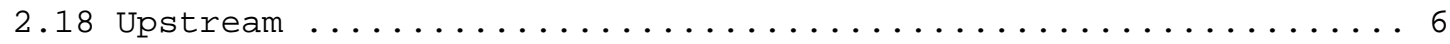

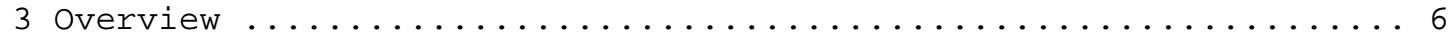

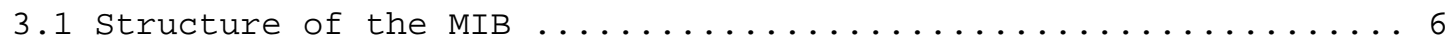

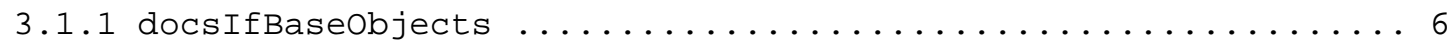

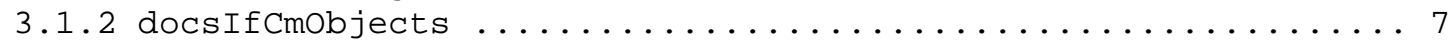

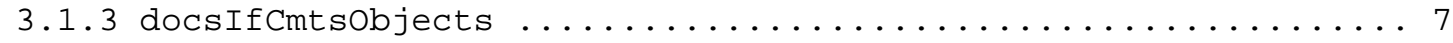

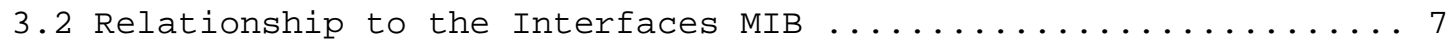

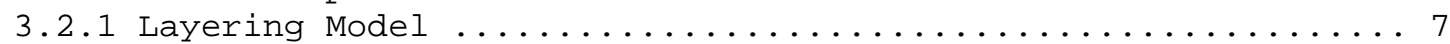

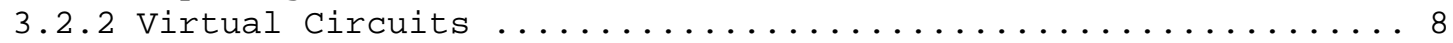

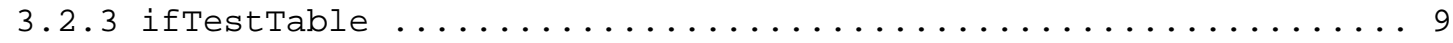

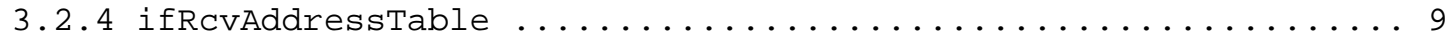

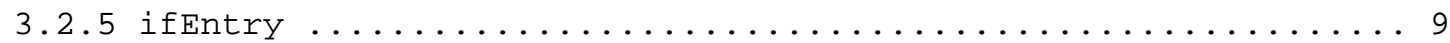

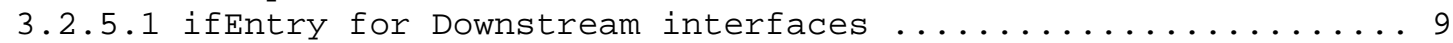

3.2.5.1.1 ifEntry for Downstream interfaces in Cable Modem

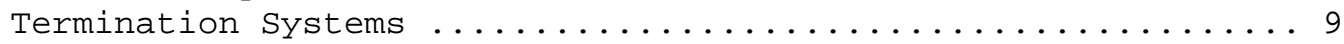

3.2.5.1.2 ifEntry for Downstream interfaces in Cable Modems ...... 11

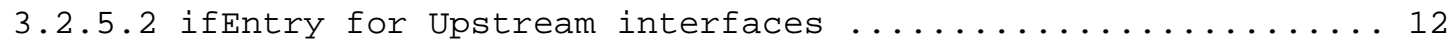

3.2.5.2.1 ifEntry for Upstream interfaces in Cable Modem

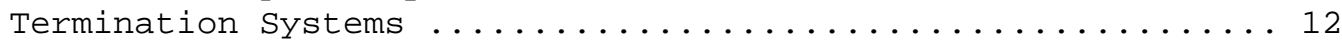

3.2.5.2.2 ifEntry for Upstream interfaces in Cable Modems ...... 14

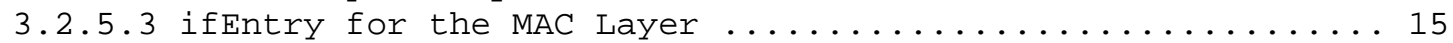

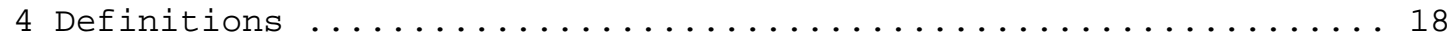

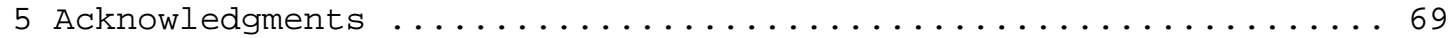

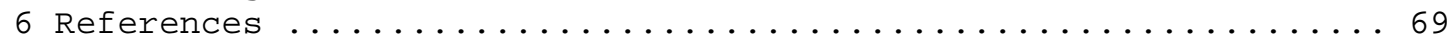

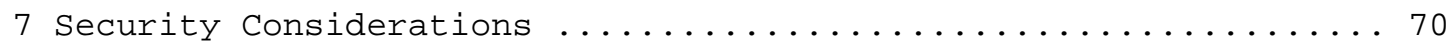

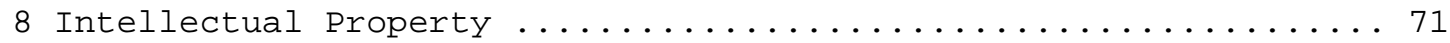

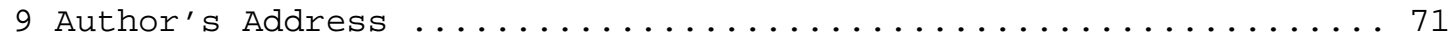

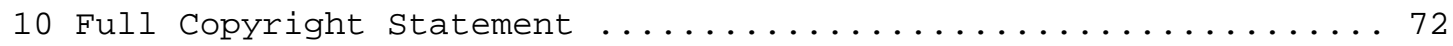


1. The SNMP Management Framework

The SNMP Management Framework presently consists of five major components:

- An overall architecture, described in RFC 2571 [1].

- Mechanisms for describing and naming objects and events for the purpose of management. The first version of this structure of Management Information (SMI) is called SMIV1 and described in STD 16, RFC 1155 [2], STD 16, RFC 1212 [3] and RFC 1215 [4]. The second version, called SMIv2, is described in STD 58, RFC 2578 [5], STD 58, RFC 2579 [6] and STD 58, RFC 2580 [7].

- Message protocols for transferring management information. The first version of the SNMP message protocol is called SNMPV1 and described in RFC 1157 [8]. A second version of the SNMP message protocol, which is not an Internet standards track protocol, is called SNMPv2c and described in RFC 1901 [9] and RFC 1906 [10]. The third version of the message protocol is called SNMPv3 and described in RFC 1906 [10], RFC 2572 [11] and RFC 2574 [12].

- Protocol operations for accessing management information. The first set of protocol operations and associated PDU formats is described in STD 15, RFC 1157 [8]. A second set of protocol operations and associated PDU formats is described in RFC 1905 [13].

- A set of fundamental applications described in RFC 2573 [14] and the view-based access control mechanism described in RFC 2575 [15].

Managed objects are accessed via a virtual information store, termed the Management Information Base or MIB. Objects in the MIB are defined using the mechanisms defined in the SMI.

This memo specifies a MIB module that is compliant to the SMIV2. A MIB conforming to the SMIv1 can be produced through the appropriate translations. The resulting translated MIB MUST be semantically equivalent, except where objects or events are omitted because no translation is possible (use of Counter64). Some machine readable information in SMIV2 will be converted into textual descriptions in SMIv1 during the translation process. However, this loss of machine readable information is not considered to change the semantics of the MIB. 


\section{Glossary}

The terms in this document are derived either from normal cable system usage, or from the documents associated with the Data Over Cable Service Interface Specification process.

\subsection{CATV}

Originally "Community Antenna Television", now used to refer to any cable or hybrid fiber and cable system used to deliver video signals to a community.

\subsection{Channel}

A specific frequency allocation with an RF medium, specified by channel width in Hertz (cycles per second) and by center frequency. Within the US Cable systems, upstream channels are generally allocated from the $5-42 \mathrm{MHz}$ range while down stream channels are generally allocated from the $50-750 \mathrm{MHz}$ range depending on the capabilities of the given system. The typical broadcast channel width in the US is $6 \mathrm{MHz}$. Upstream channel widths for DoCSIS vary.

2.3. CM Cable Modem.

A CM acts as a "slave" station in a DoCSIS compliant cable data system.

\subsection{CMTS Cable Modem Termination system.}

A generic term covering a cable bridge or cable router in a head-end. A CMTS acts as the master station in a DOCSIS compliant cable data system. It is the only station that transmits downstream, and it controls the scheduling of upstream transmissions by its associated CMs.

\subsection{Codeword}

See [16]. A characteristic of the Foward Error Correction scheme used above the RF media layer.

2.6. Data Packet

The payload portion of the MAC Packet.

2.7. $\mathrm{dBmV}$

Decibel relative to one milli-volt. A measure of RF power. 


\subsection{DOCSIS}

"Data Over Cable Interface Specification". A term referring to the ITU-T J.112 Annex B standard for cable modem systems [20].

\subsection{Downstream}

The direction from the head-end towards the subscriber.

2.10. Head-end

The origination point in most cable systems of the subscriber video signals.

2.11. MAC Packet

A DOCSIS PDU.

\subsection{MCNS}

"Multimedia Cable Network System". Generally replaced in usage by DOCSIS.

2.13. Mini-slot

See [16]. In general, an interval of time which is allocated by the CMTS to a given CM for that CM to transmit in an upstream direction.

2.14. QPSK Quadrature Phase Shift Keying.

A particular modulation scheme on an RF medium. See [19].

2.15. QAM Quadrature Amplitude Modulation.

A particular modulation scheme on on RF medium. Usually expressed with a number indicating the size of the modulation constellation (e.g. 16 QAM). See [19], or any other book on digital communications over RF for a complete explanation of this.

2.16. $\mathrm{RF}$

Radio Frequency •

2.17. Symbol-times

See [16]. A characteristic of the RF modulation scheme. 


\subsection{Upstream}

The direction from the subscriber towards the head-end.

\section{Overview}

This MIB provides a set of objects required for the management of MCNS/DOCSIS compliant Cable Modem (CM) and Cable Modem Termination System (CMTS) RF interfaces. The specification is derived in part from the parameters and protocols described in DoCSIS Radio Frequency Interface Specification [16].

\subsection{Structure of the MIB}

This MIB is structured as three groups:

- Management information pertinent to both Cable Modems (CM) and Cable Modem Termination Systems (CMTS) (docsIfBaseobjects).

- Management information pertinent to Cable Modems only (docsIfCmObjects).

- Management information pertinent to Cable Modem Termination systems only (docsifCmtsobjects).

Tables within each of these groups group objects functionally - e.g. Quality of Service, Channel characteristics, MAC layer management, etc. Rows created automatically (e.g. by the device according to the hardware configuration) may and generally will have a mixture of configuration and status objects within them. Rows that are meant to be created by the management station are generally restricted to configuration (read-create) objects.

\subsection{1. docsifBaseobjects}

docsIfDownstreamchannelTable - This table describes the active downstream channels for a CMTS and the received downstream channel for a CM.

docsifUpstreamchannelTable - This table describes the active upstream channels for a a CMTS and the current upstream transmission channel for a CM.

docsIfQosProfileTable - This table describes the valid Quality of Service service profiles for the cable data system.

docsIfSignalQualityTable - This table is used to monitor RF signal quality characteristics of received signals. 


\subsection{2. docsIfCmObjects}

docsifCmMacTable - This table is used to monitor the DOCSIS MAC interface and can be considered an extension to the ifEntry.

docsIfCmServiceTable - This table describes the upstream service queues available at this CM. There is a comparable table at the CMTS, docsifcmtsserviceEntry, which describes the service queues from the point of view of the CMTS.

3.1.3. docsIfCmtsObjects

docsifCmtstatustable - This table provides a set of aggregated counters which roll-up values and events that occur on the underlying sub-interfaces.

docsIfCmtsCmStatusTable - This table is used to hold information about known (e.g. registered) cable modems on the system serviced by this CMTS.

docsifCmtsServiceEntry - This table provides access to the information related to upstream service queues.

docsIfCmtsModulationTable - This table allows control over the modulation profiles for RF channels associated with this CMTS.

docsifcmtsmactocmTable - This table allows fast access into the docsifCmtsCmTable via a MAC address (of the CM) interface.

\subsection{Relationship to the Interfaces MIB}

This section clarifies the relationship of this MIB to the Interfaces MIB [17]. Several areas of correlation are addressed in the following subsections. The implementor is referred to the Interfaces MIB document in order to understand the general intent of these areas.

\subsubsection{Layering Model}

An instance of ifEntry exists for each RF Downstream interface, for each RF Upstream interface, and for each RF MAC layer. The ifstackTable [17] MUST be implemented to identify relationships among sub-interfaces. 
The following example illustrates a MAC interface with one downstream and two upstream channels.

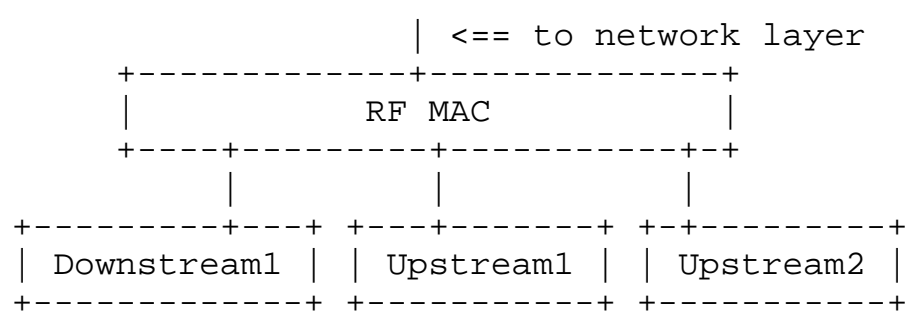

As can be seen from this example, the RF MAC interface is layered on top of the downstream and upstream interfaces.

In this example, the assignment of index values could be as follows:

$$
\text { ifIndex iftype } \quad \text { Description }
$$

1 docsCableMaclayer(127) CATV MAC Layer

2 docsCableDownstream(128) CATV Downstream interface

3 docsCableUpstream(129) CATV Upstream interface

4 docsCableUpstream(129) CATV Upstream interface

The corresponding ifstack entries would then be:

$$
\begin{array}{|c|c|}
\text { IfStackHigherLayer } & \text { ifStackLowerLayer } \\
0 & 1 \\
1 & 2 \\
1 & 3 \\
2 & 4 \\
3 & 0 \\
4 & 0 \\
& 0
\end{array}
$$

The same interface model can also be used in Telephony or Telco Return systems. A pure Telco Return system (Cable Modem as well as Cable Modem Termination System) would not have upstream, but only downstream cable channels. Systems supporting both Telco Return and cable upstream channels can use the above model without modification.

Telco Return Upstream channel(s) are handled by the appropriate MIBs, such as PPP or Modem MIBs.

\subsubsection{Virtual Circuits}

This medium does not support virtual circuits and this area is not applicable to this MIB. 


\subsection{3. ifTestTable}

The ifTestTable is not supported by this MIB.

\subsection{4. ifRcvAddressTable}

The ifRcvAddressTable is not supported by this MIB.

\subsection{5. ifEntry}

This section documents only the differences from the requirements specified in the Interfaces MIB. See that MIB for columns omitted from the descriptions below.

3.2.5.1. ifEntry for Downstream interfaces

The ifEntry for Downstream interfaces supports the ifGeneralInformationGroup and the ifPacketGroup of the Interfaces MIB. This is an output only interface at the CMTS and all input status counters - ifIn* - will return zero. This is an input only interface at the $\mathrm{CM}$ and all output status counters - ifOut* - will return zero.

3.2.5.1.1. ifEntry for Downstream interfaces in Cable Modem Termination Systems

$\begin{aligned} & \text { ifTable } \\ & =========== \\ & \text { ifIndex }\end{aligned}$
ifType
ifSpeed
ifPhysAddress
ifAdminstatus
ifOperStatus
ifMtu

\author{
Comments

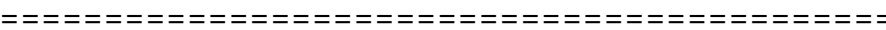 \\ Each RF Cable Downstream interface is represented \\ by an ifEntry. \\ The IANA value of docsCableDownstream(128). \\ Return the speed of this downstream channel. \\ The returned value the raw bandwidth in bits/s \\ of this interface. This is the symbol rate \\ multiplied with the number of bits per symbol. \\ Return an empty string. \\ The administrative status of this interface. \\ The current operational status of this interface. \\ The size of the largest frame which can be \\ sent on this interface, specified in octets. \\ The value includes the length of the MAC header.
}




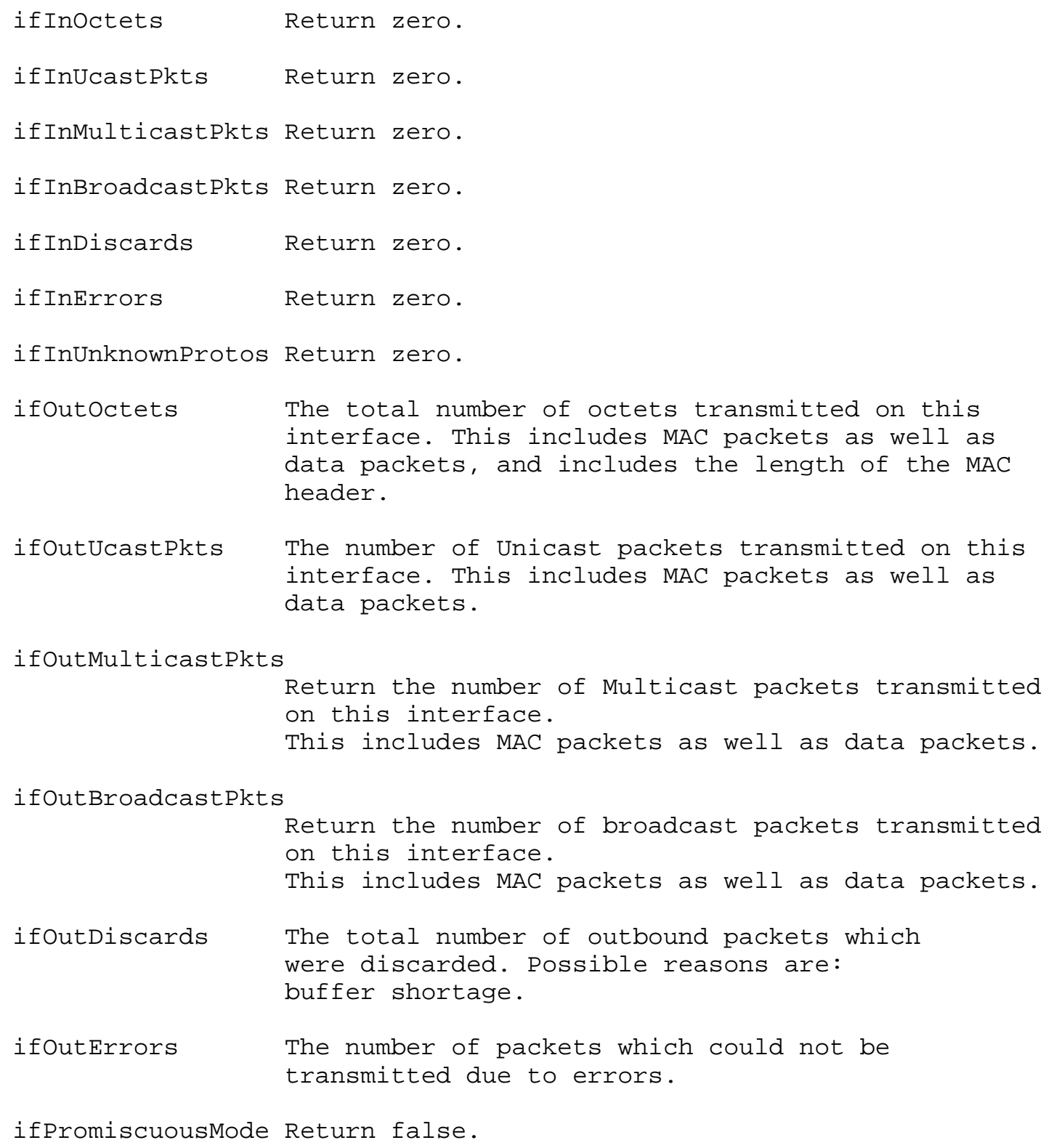




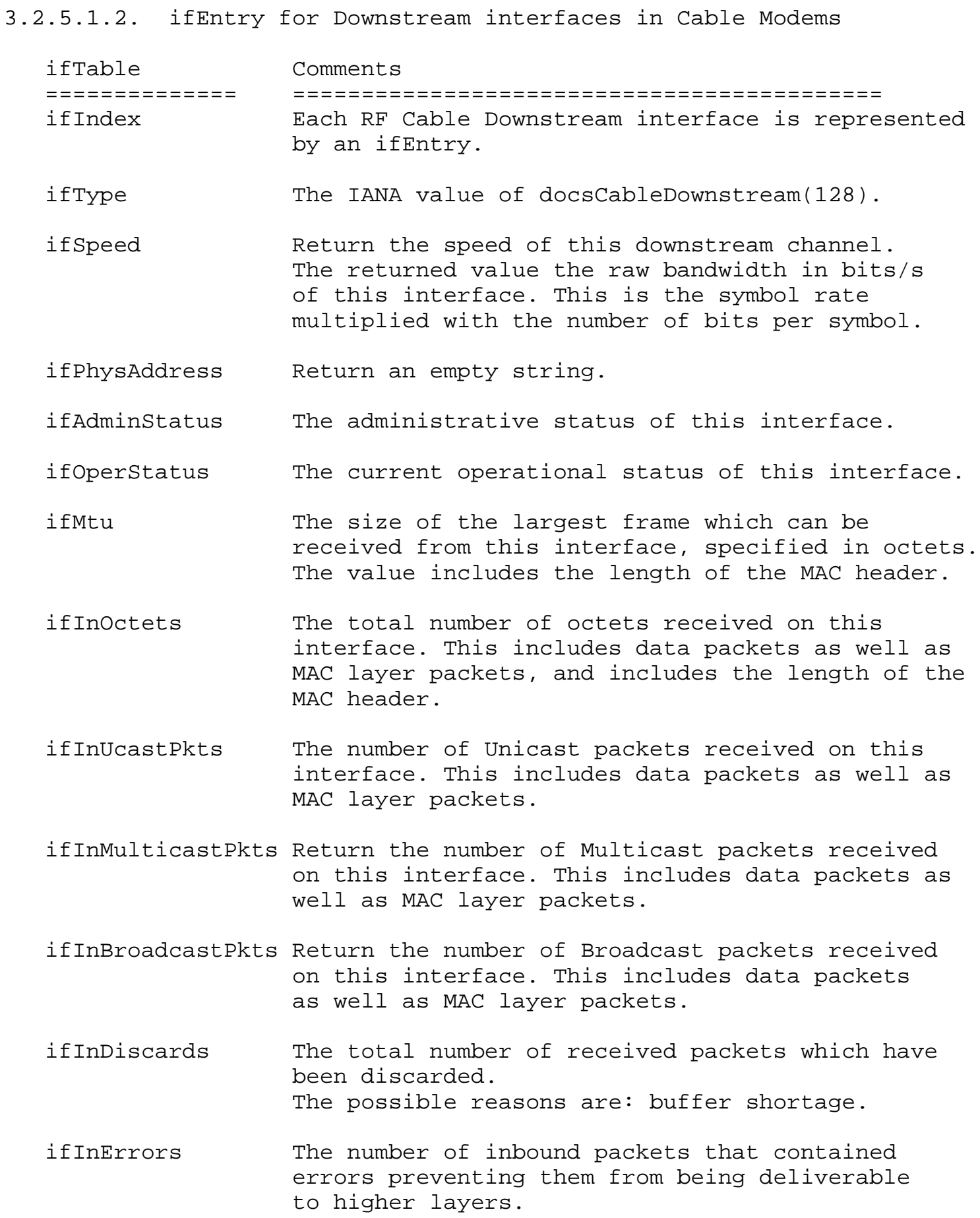




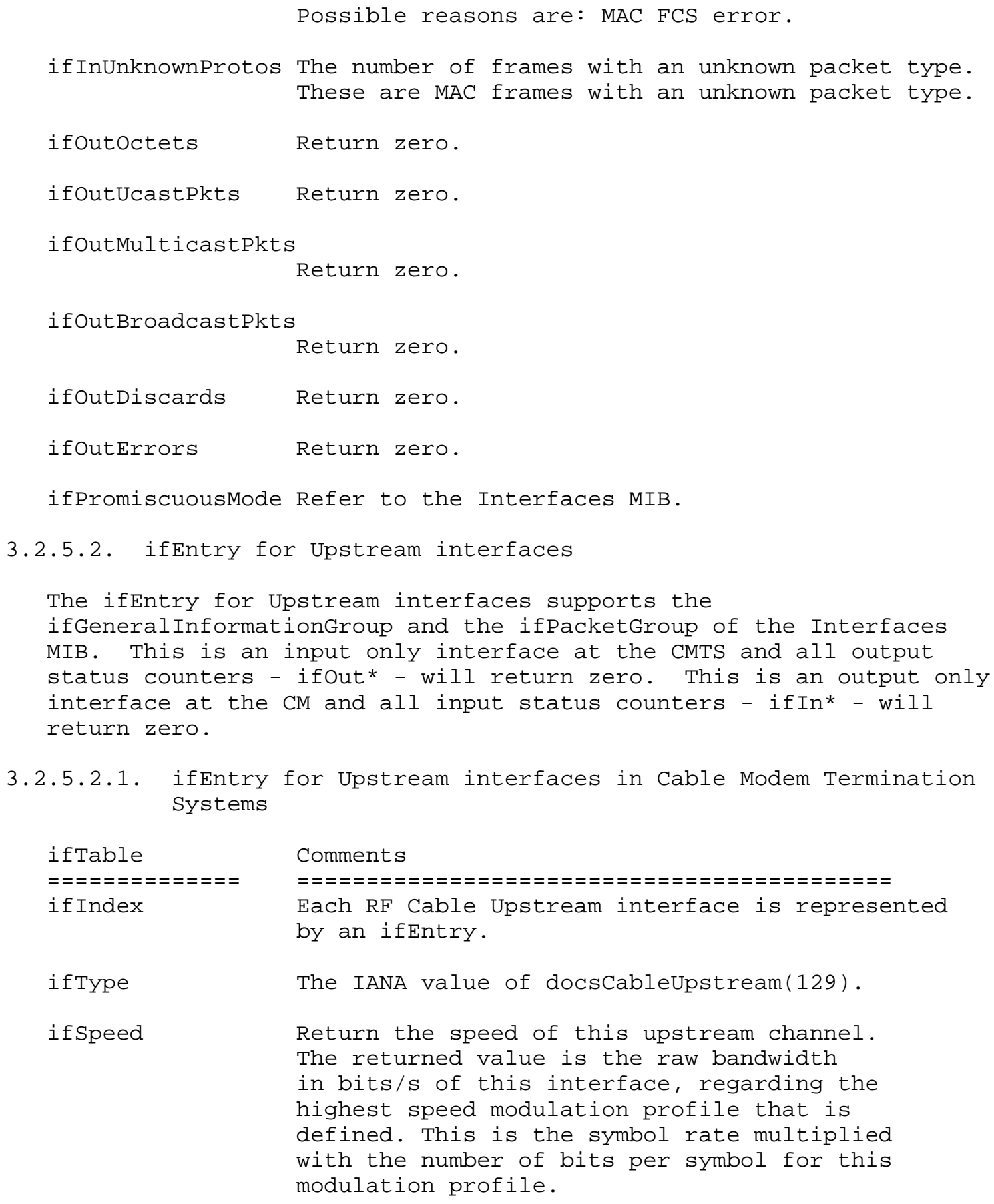

iftable

Comments

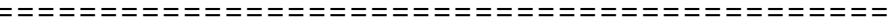
Each RF Cable Upstream interface is represented by an ifEntry.

The IANA value of docsCableUpstream(129).

Return the speed of this upstream channel. The returned value is the raw bandwidth in bits/s of this interface, regarding the highest speed modulation profile that is defined. This is the symbol rate multiplied with the number of bits per symbol for this modulation profile. 


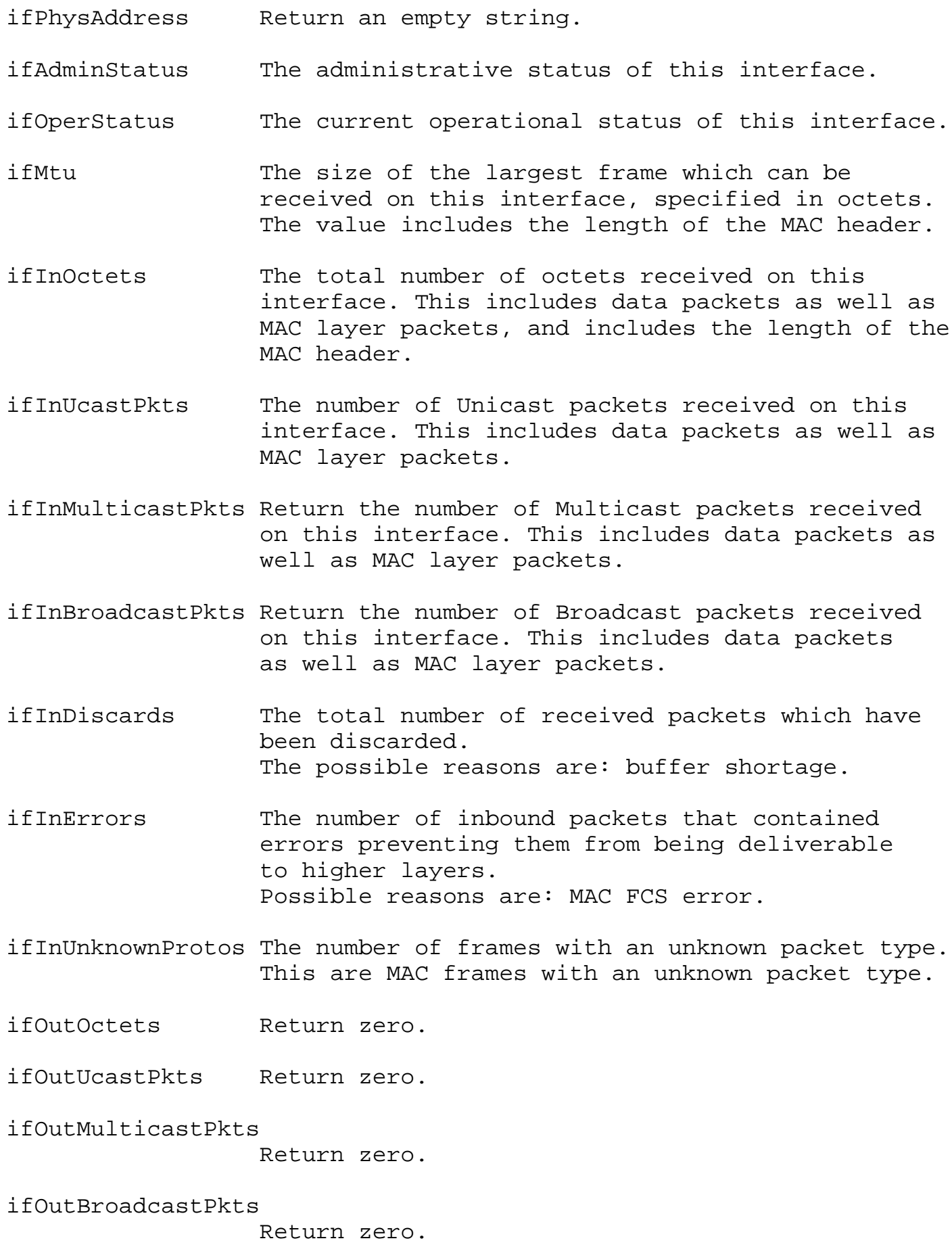




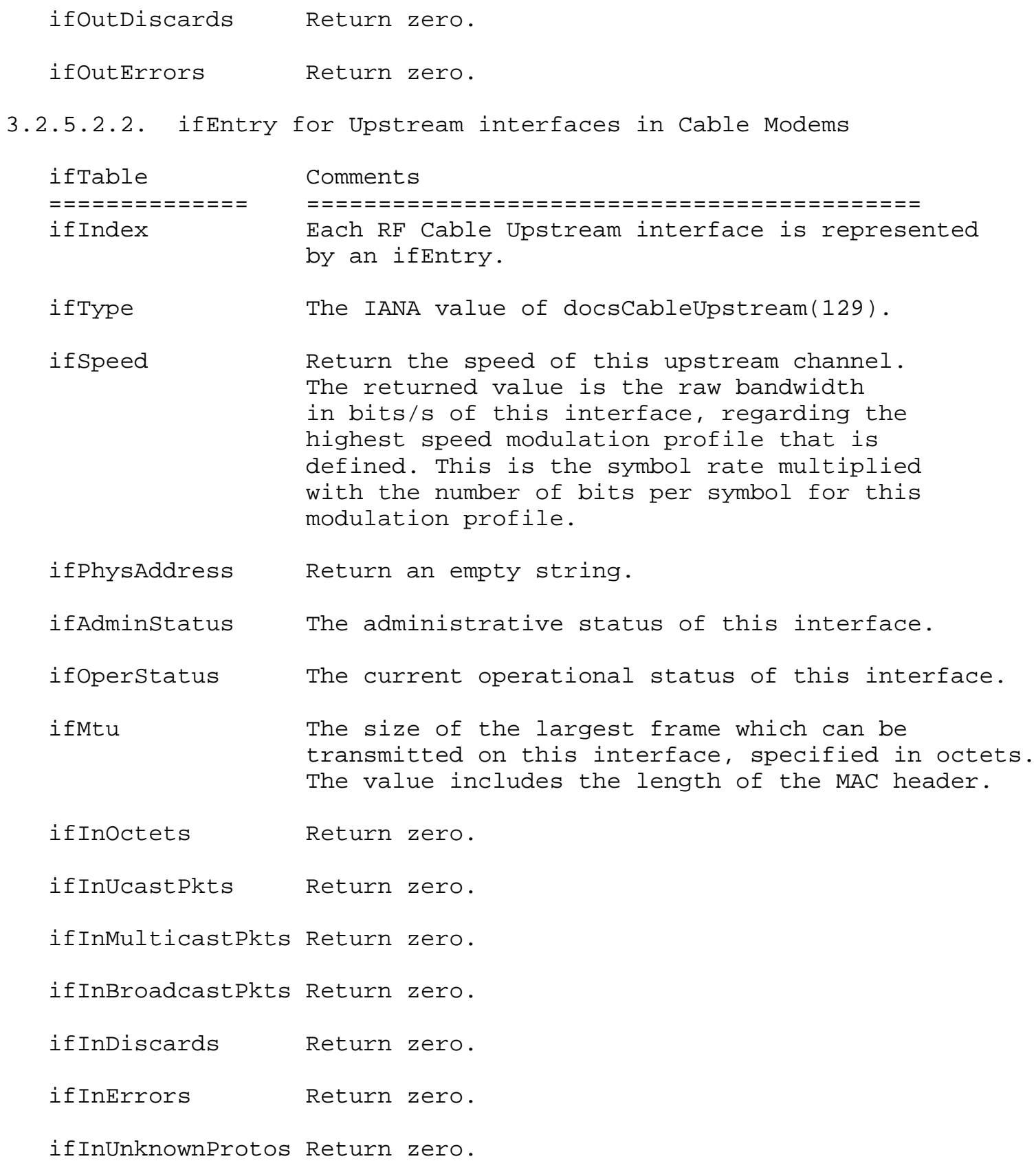




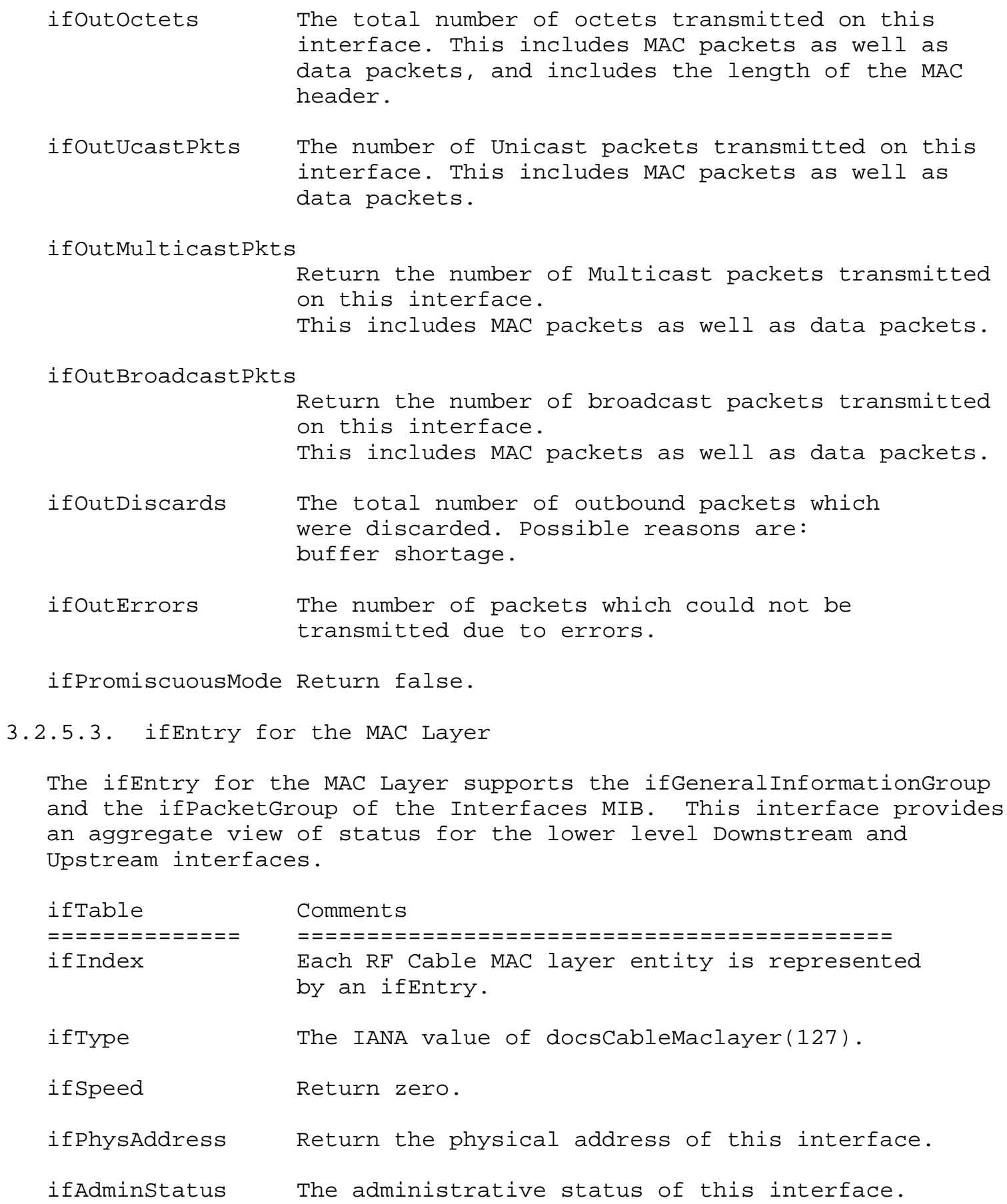




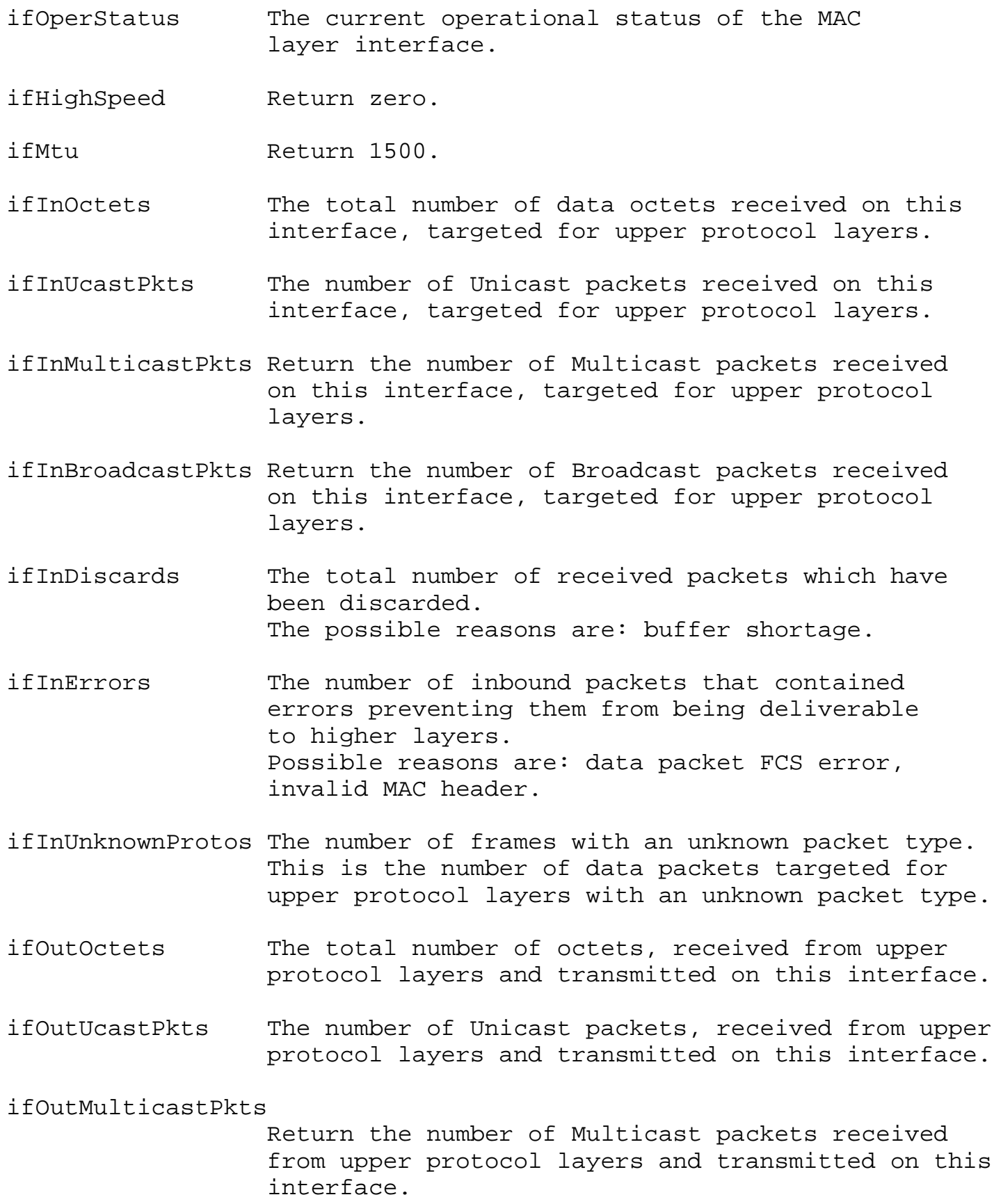




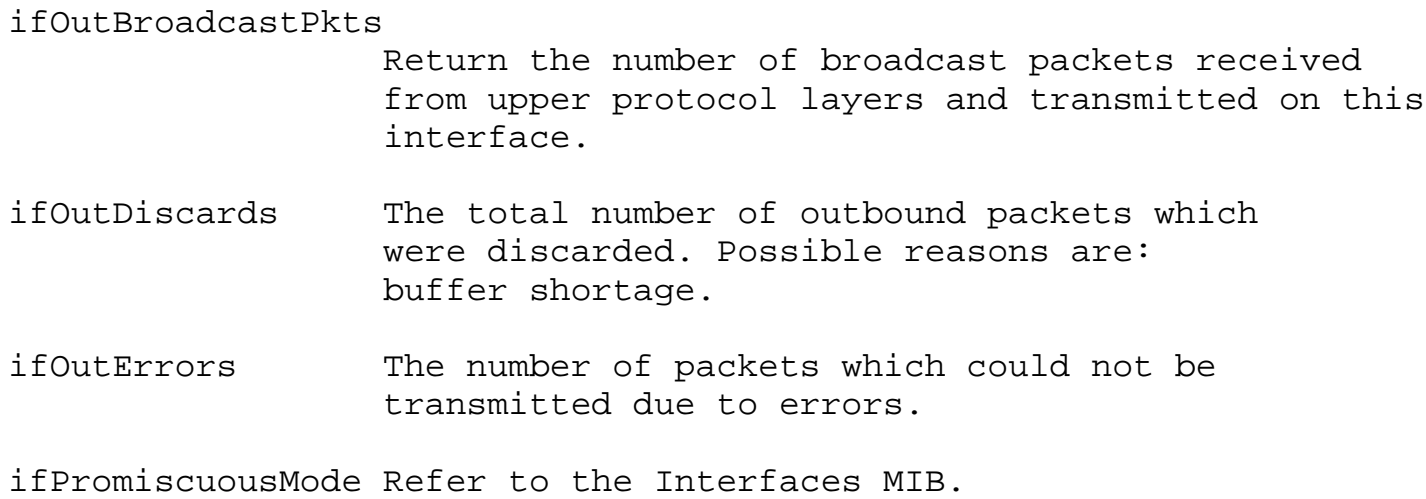


4. Definitions

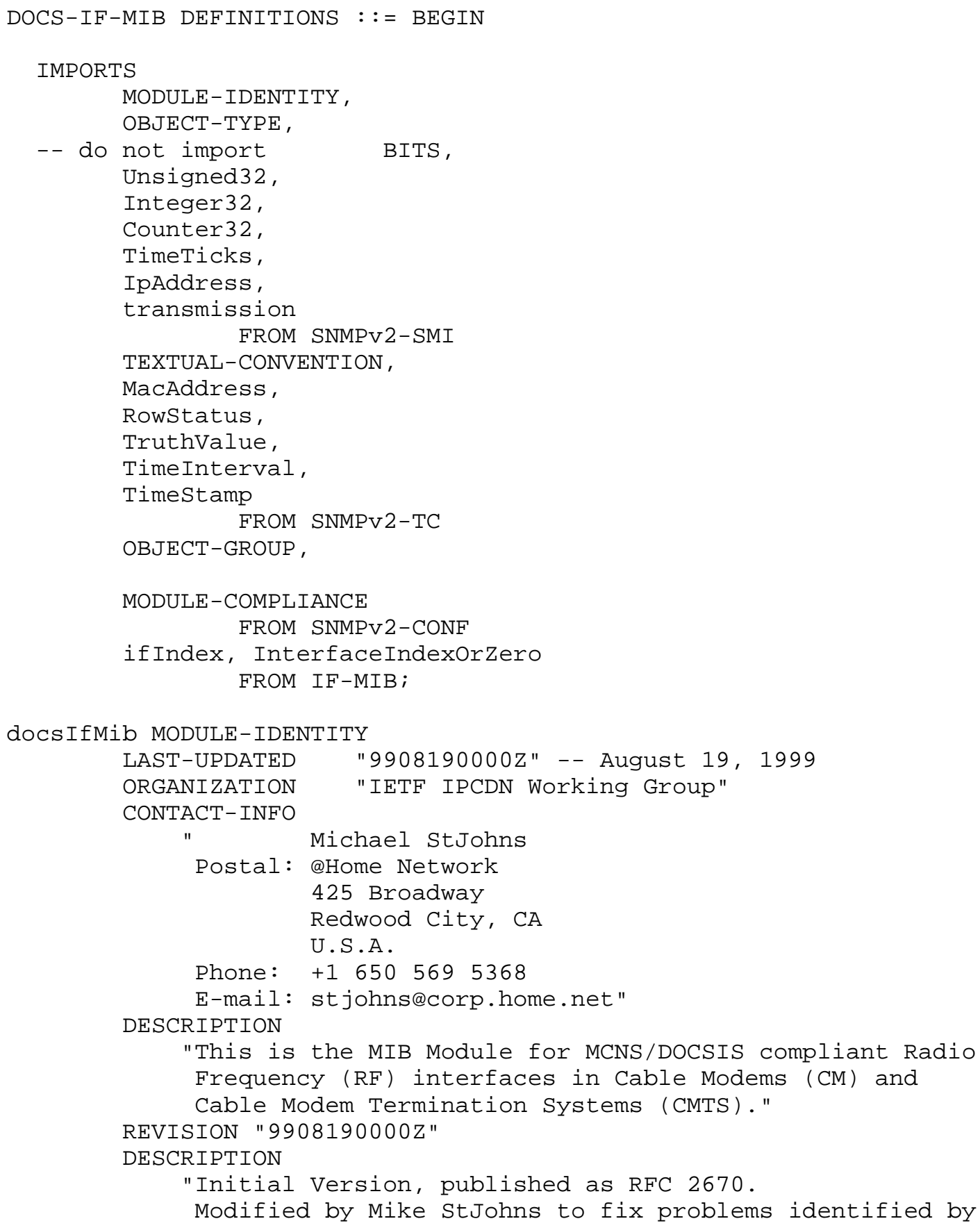




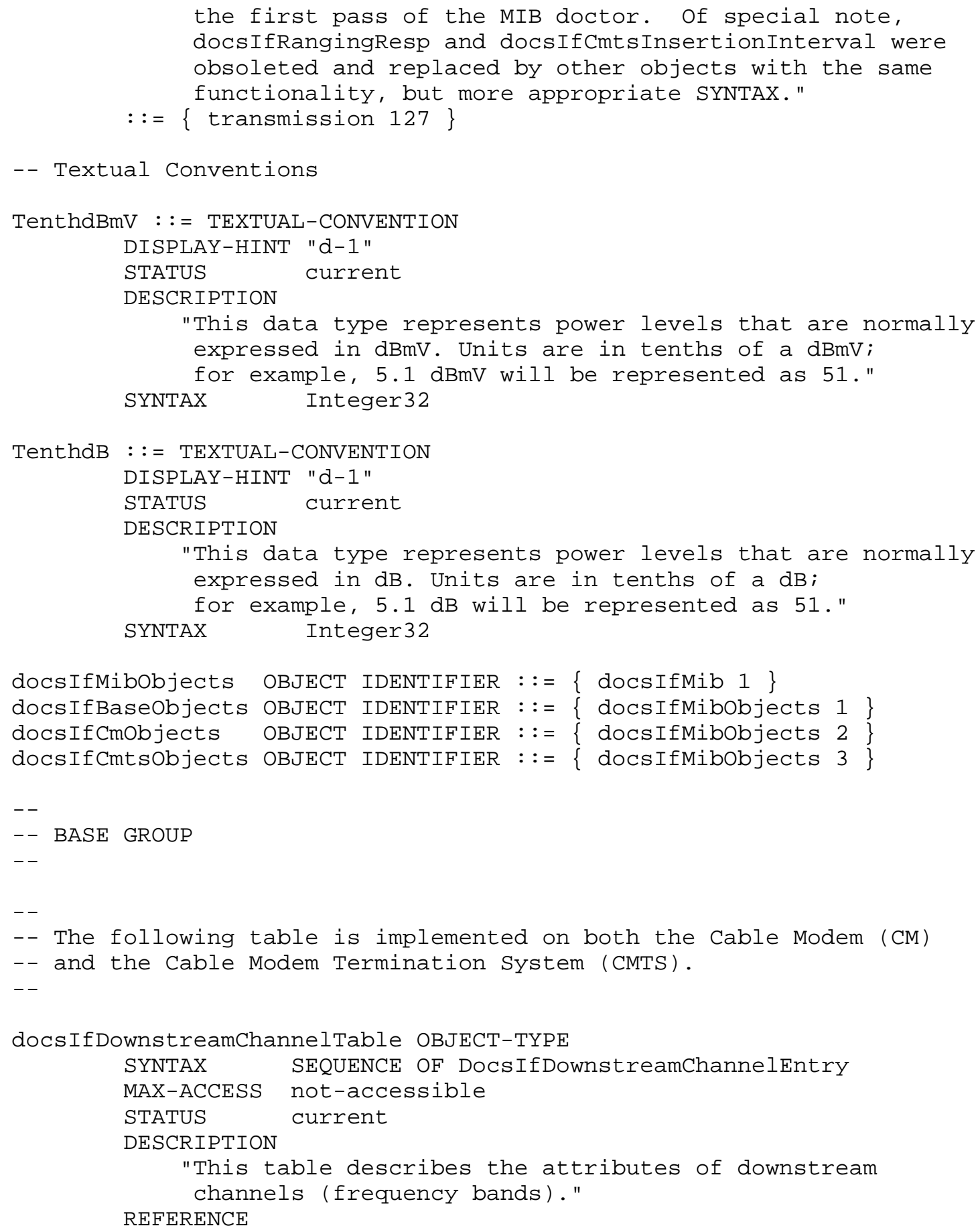




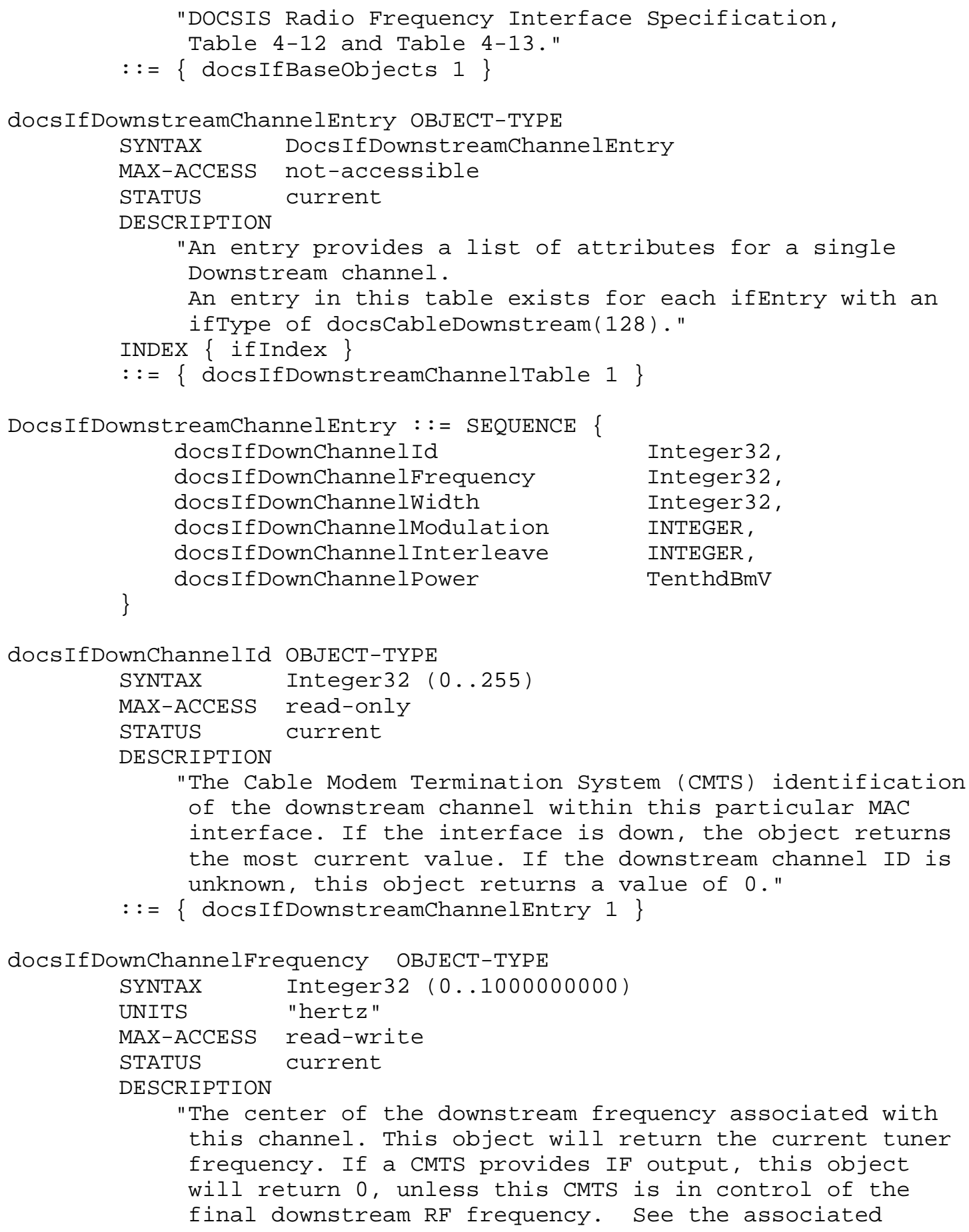




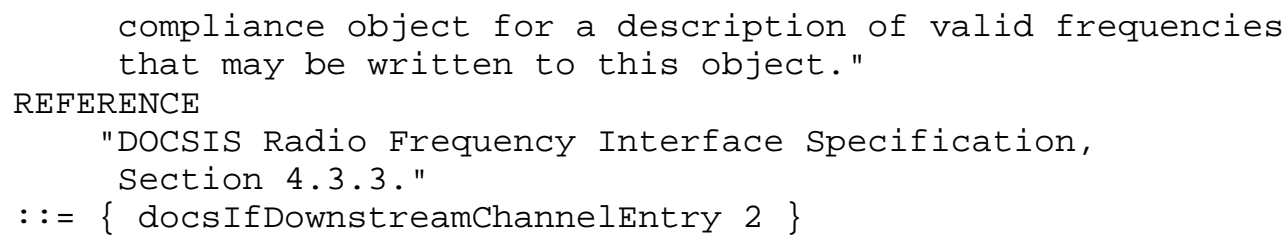




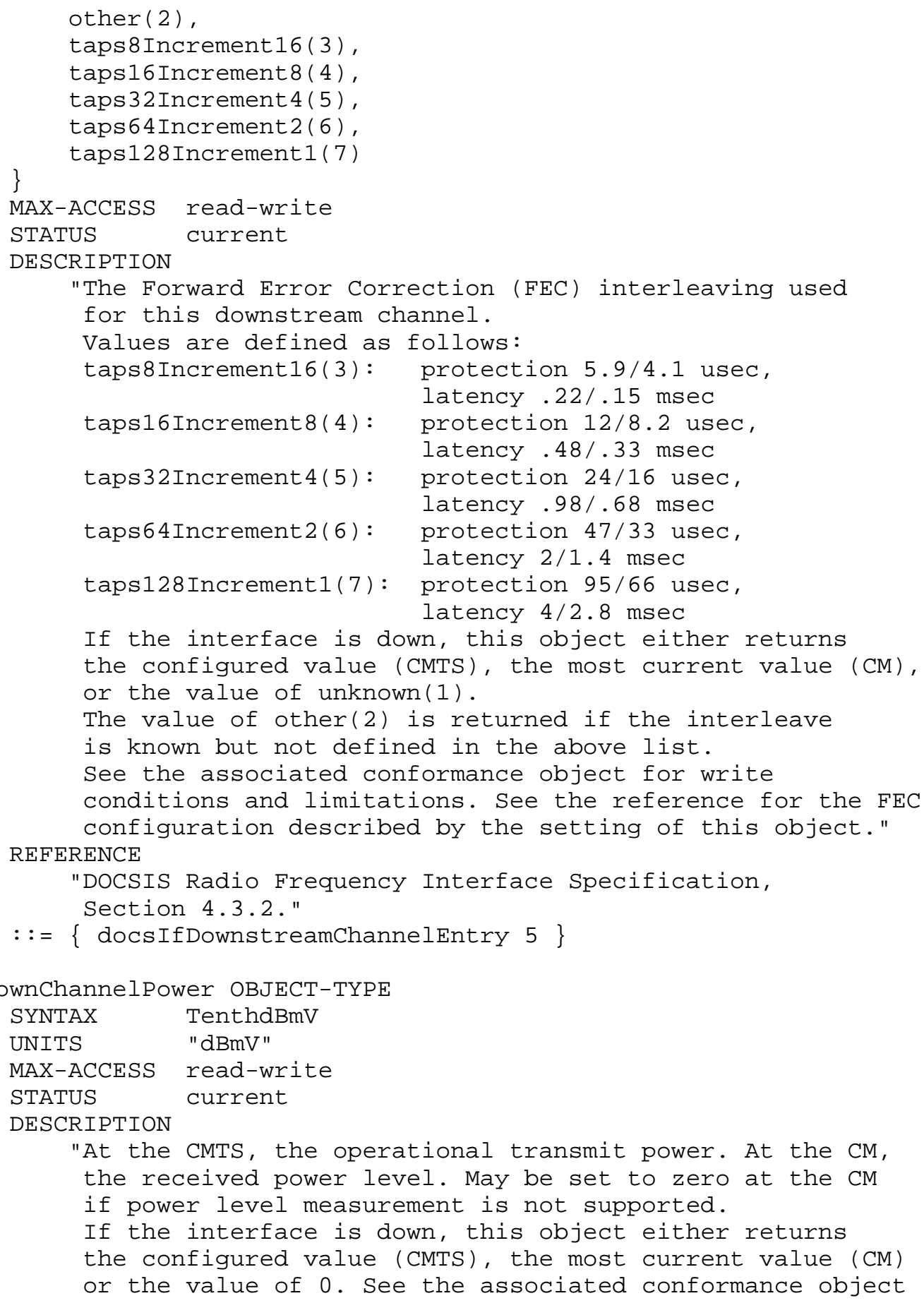




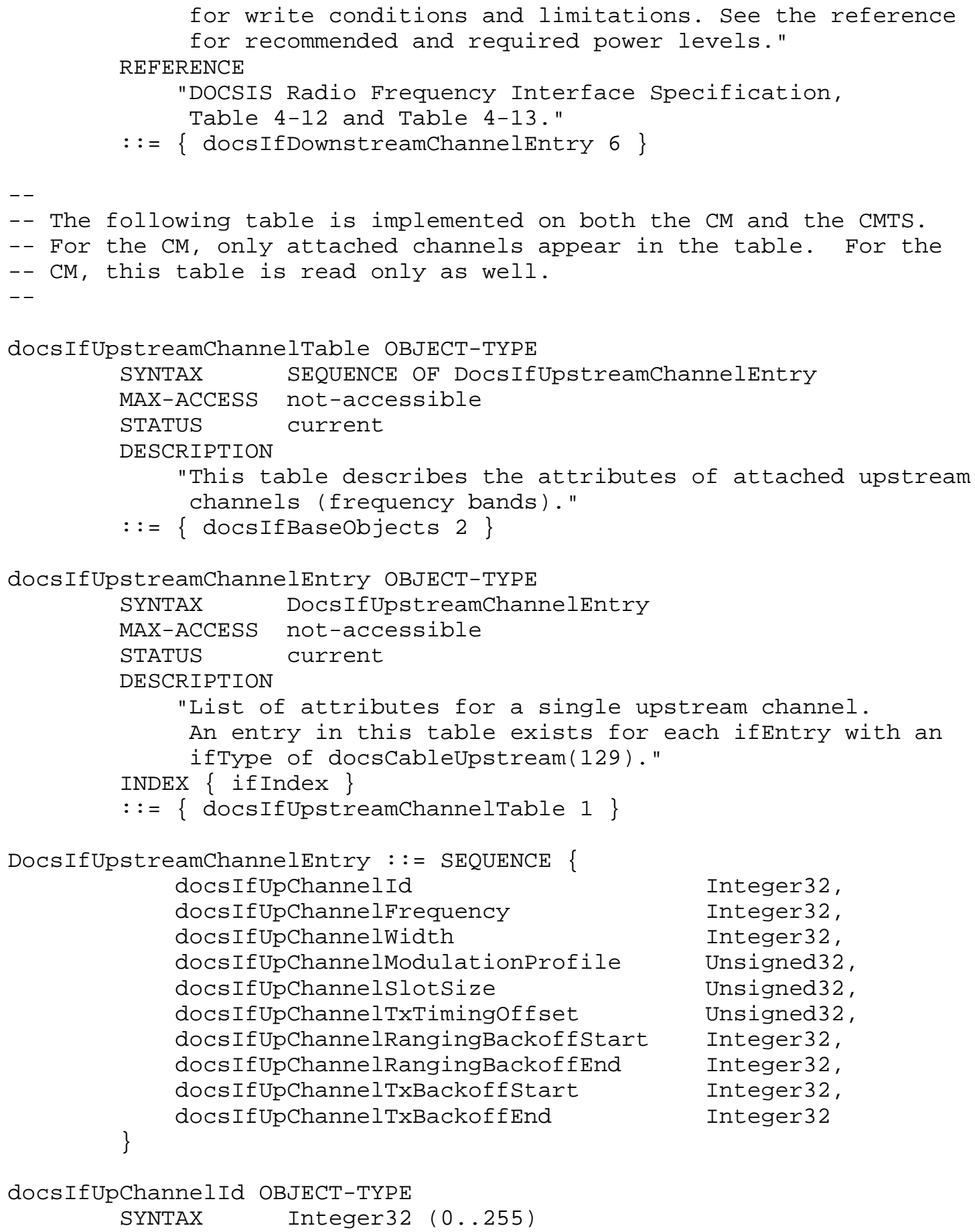




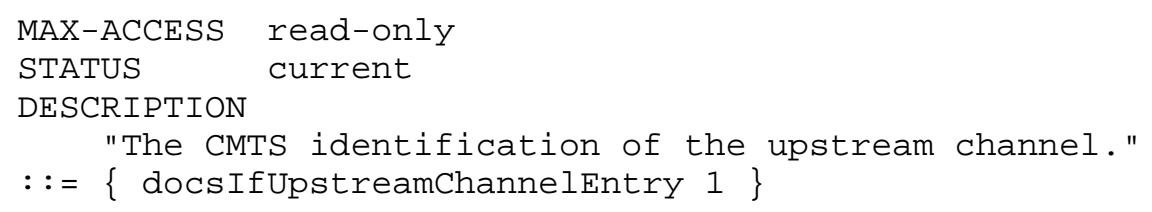




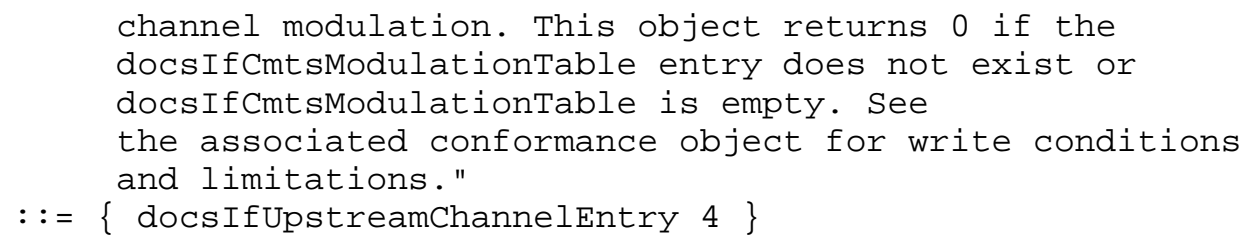




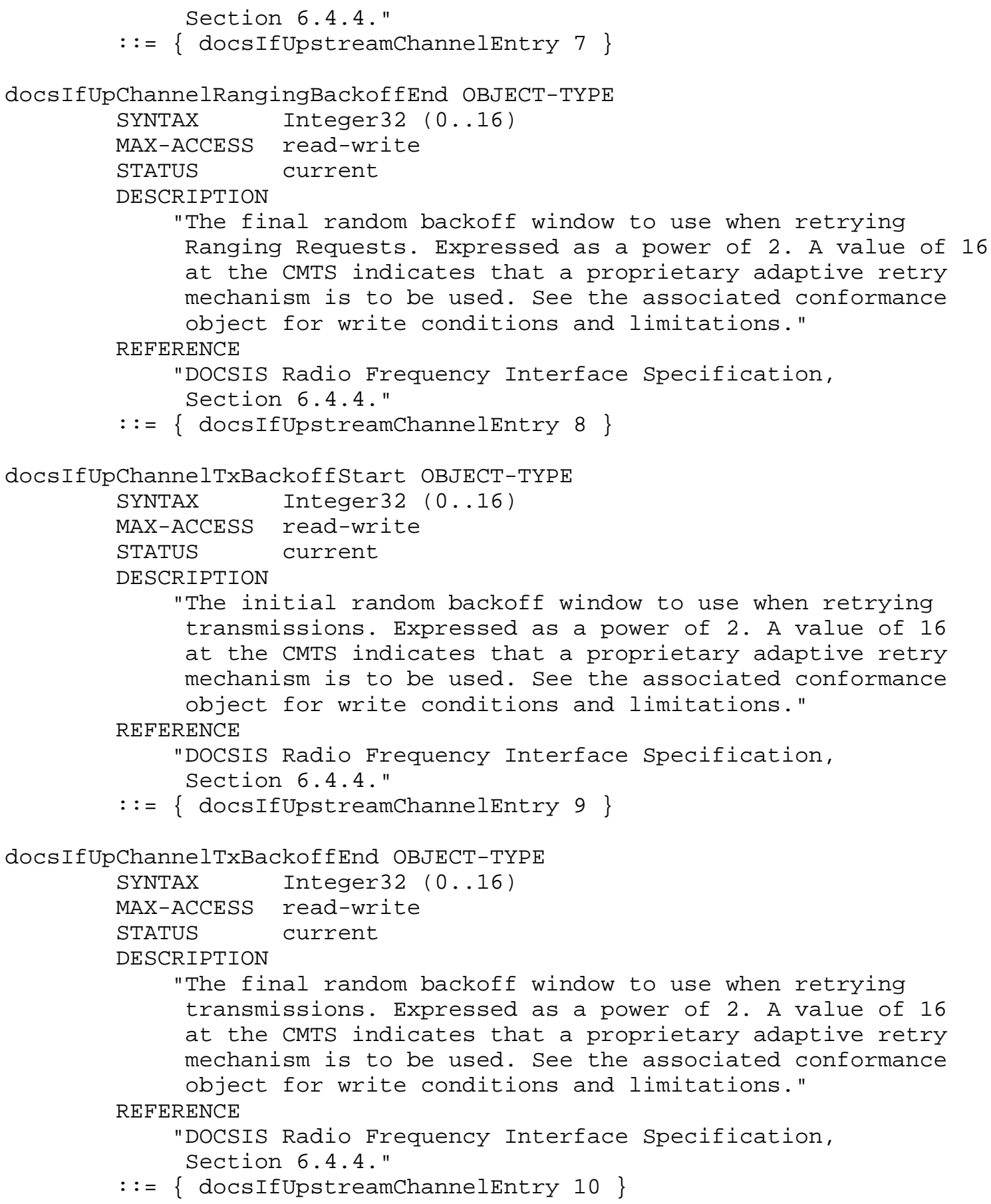




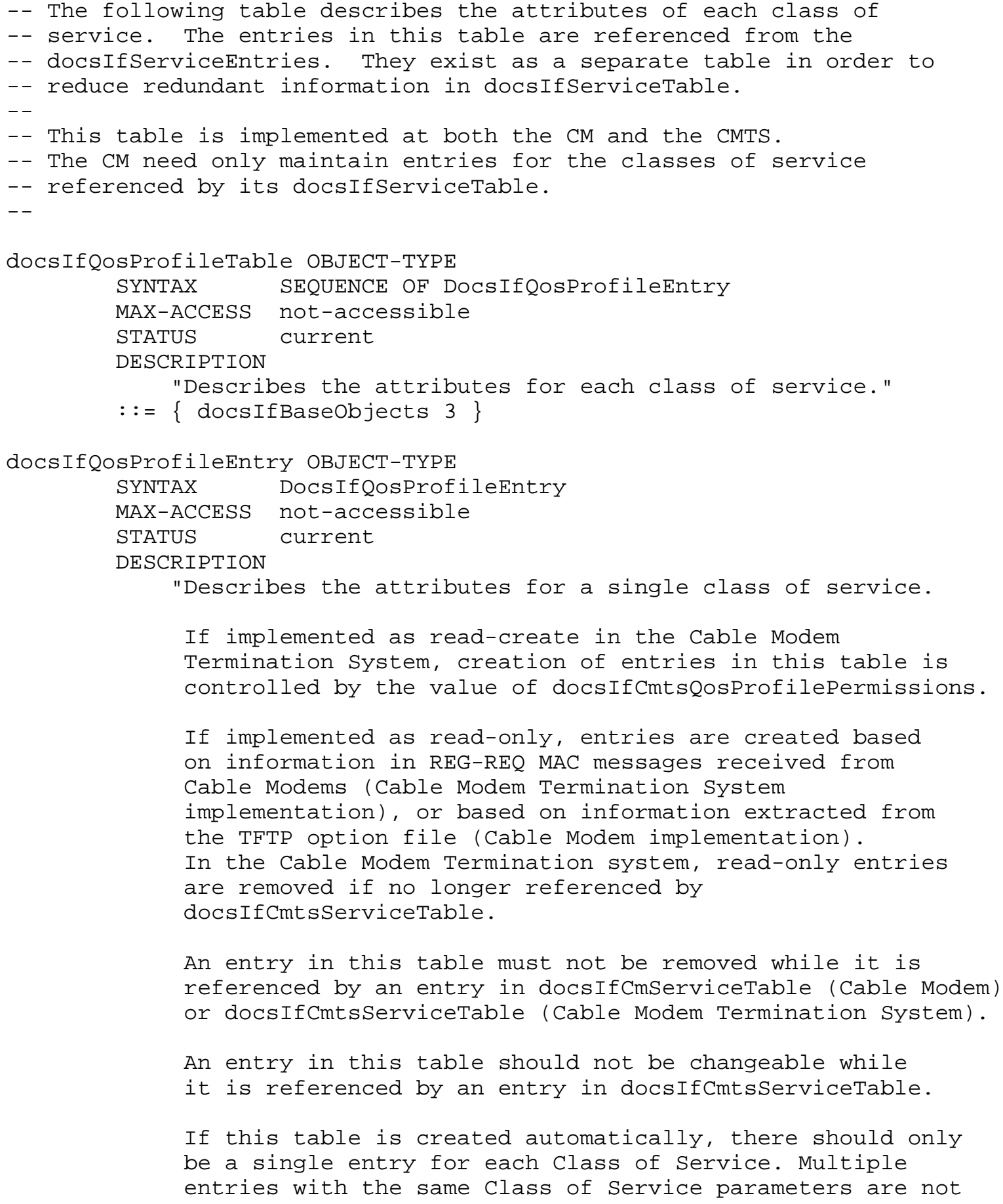




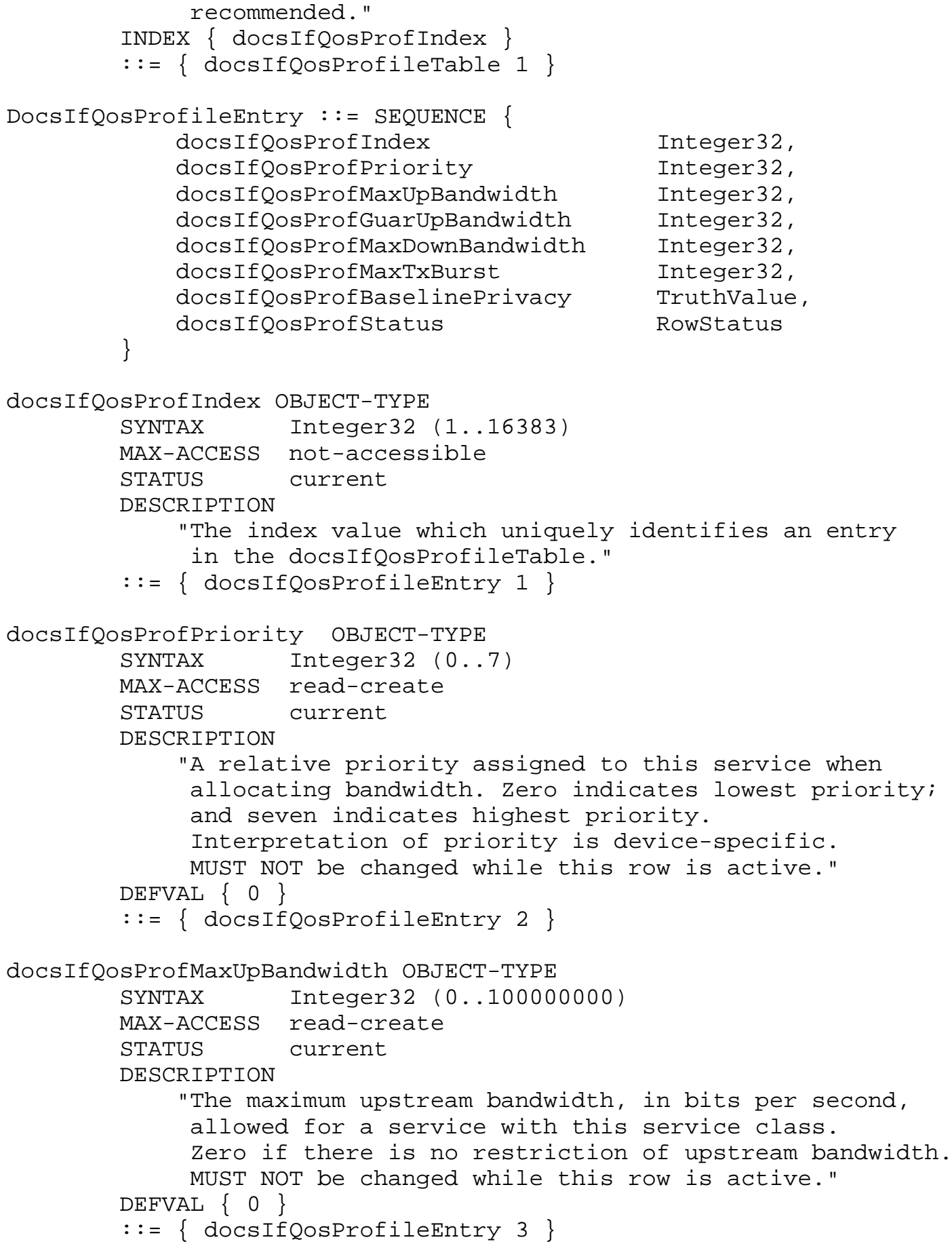




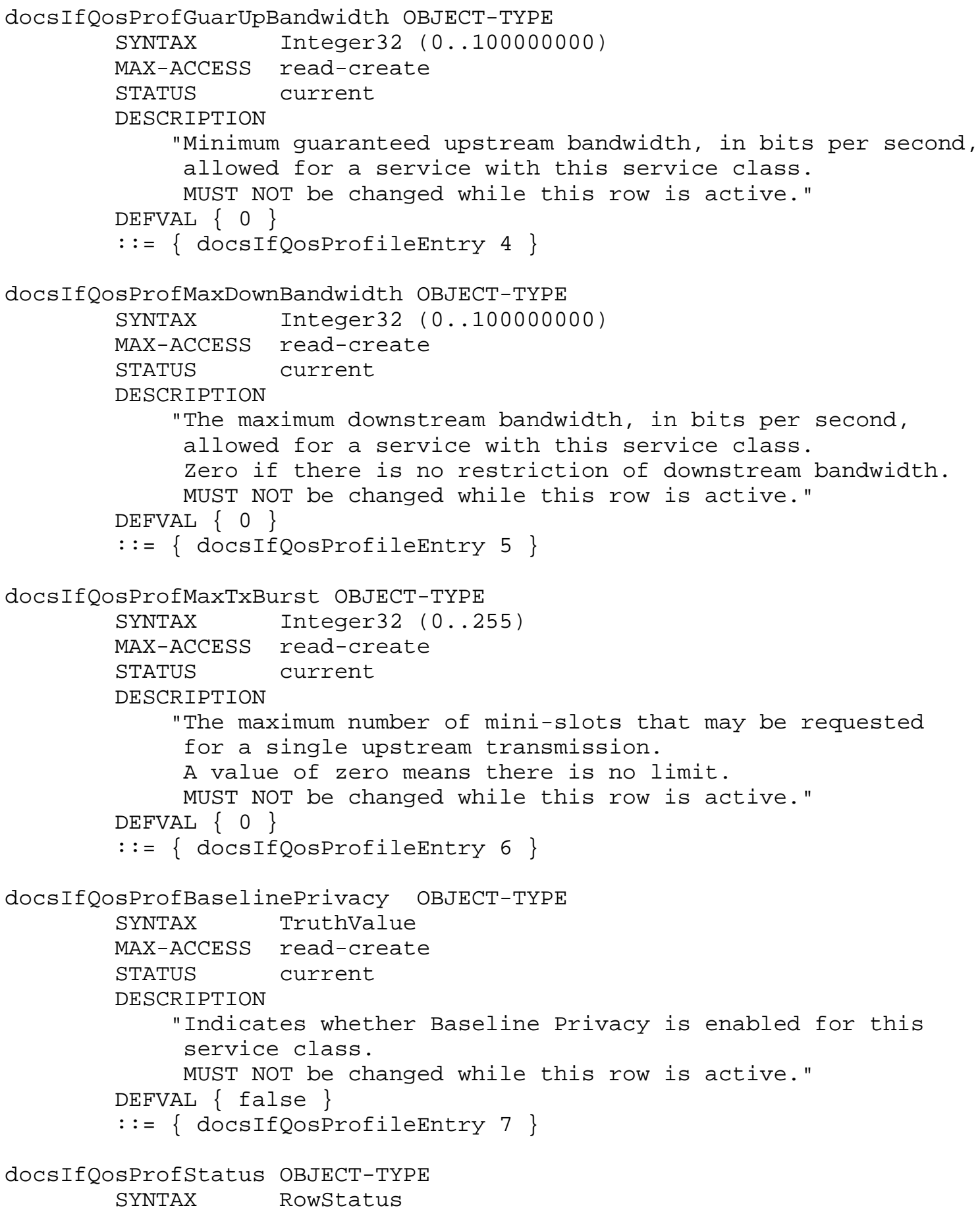




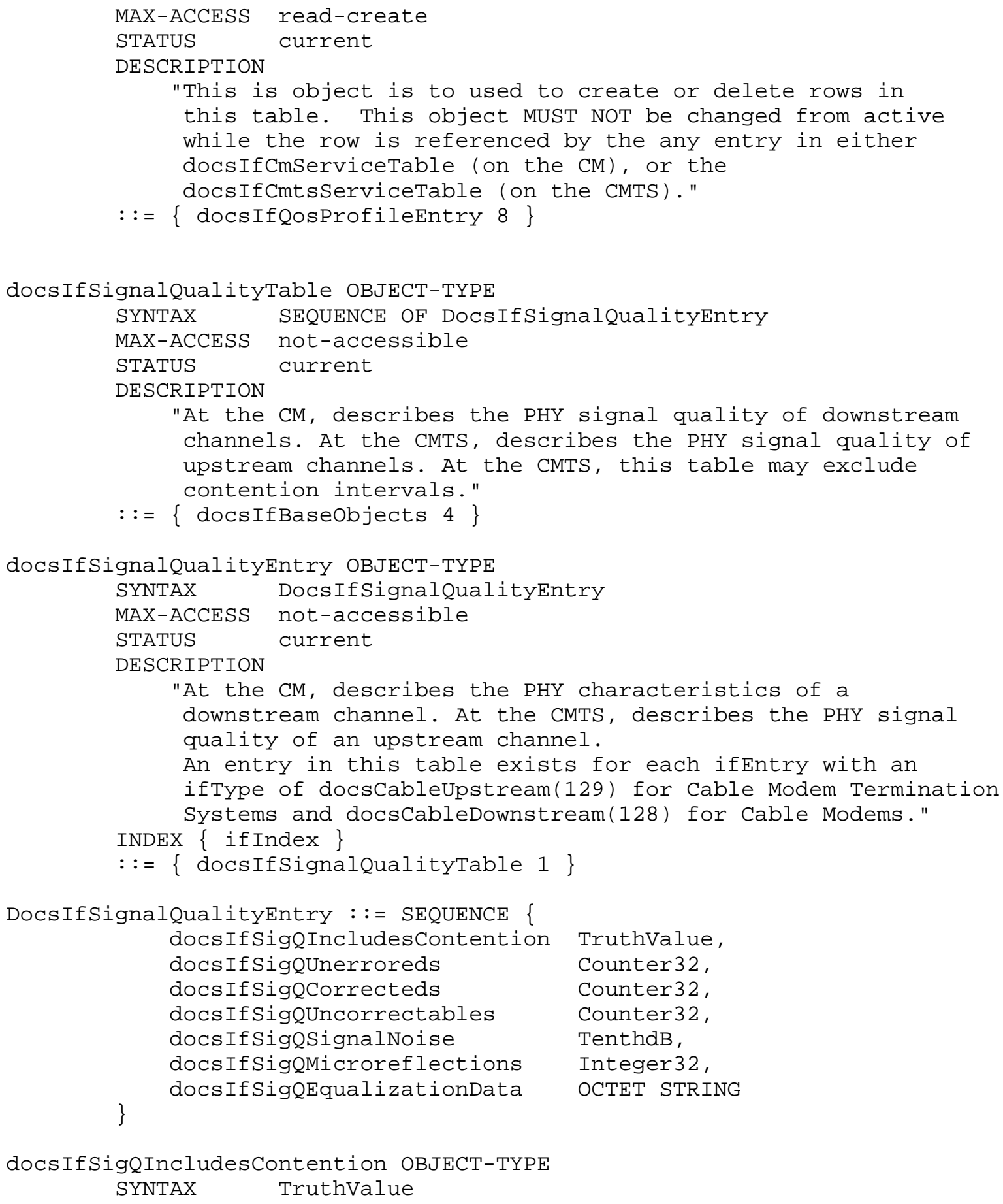




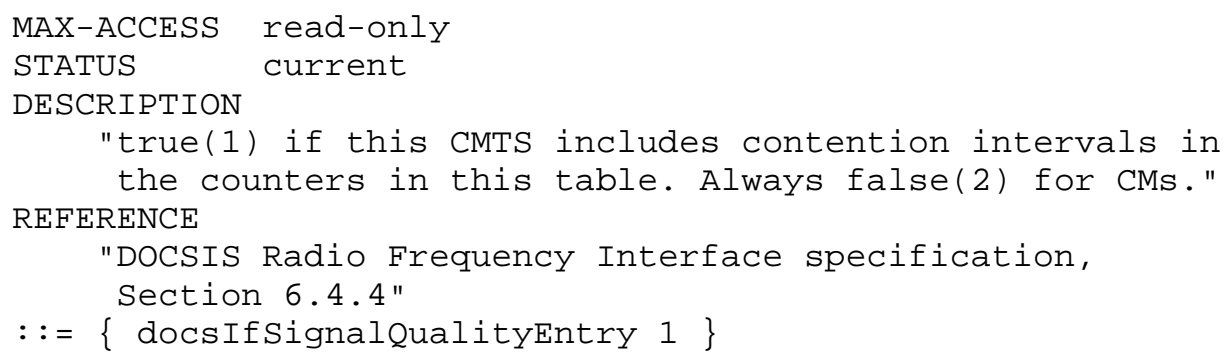




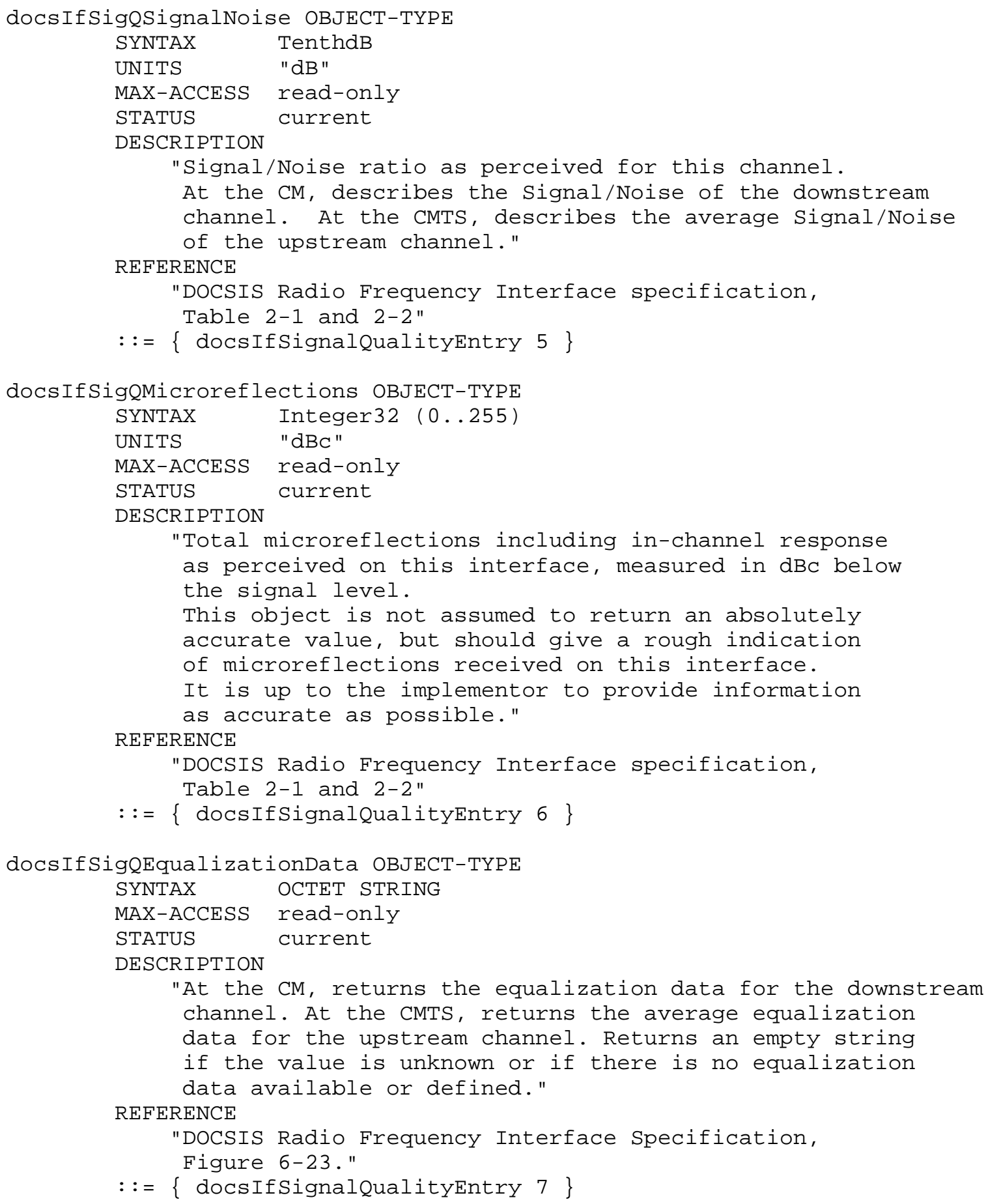

St. Johns

Standard

[Page 32] 


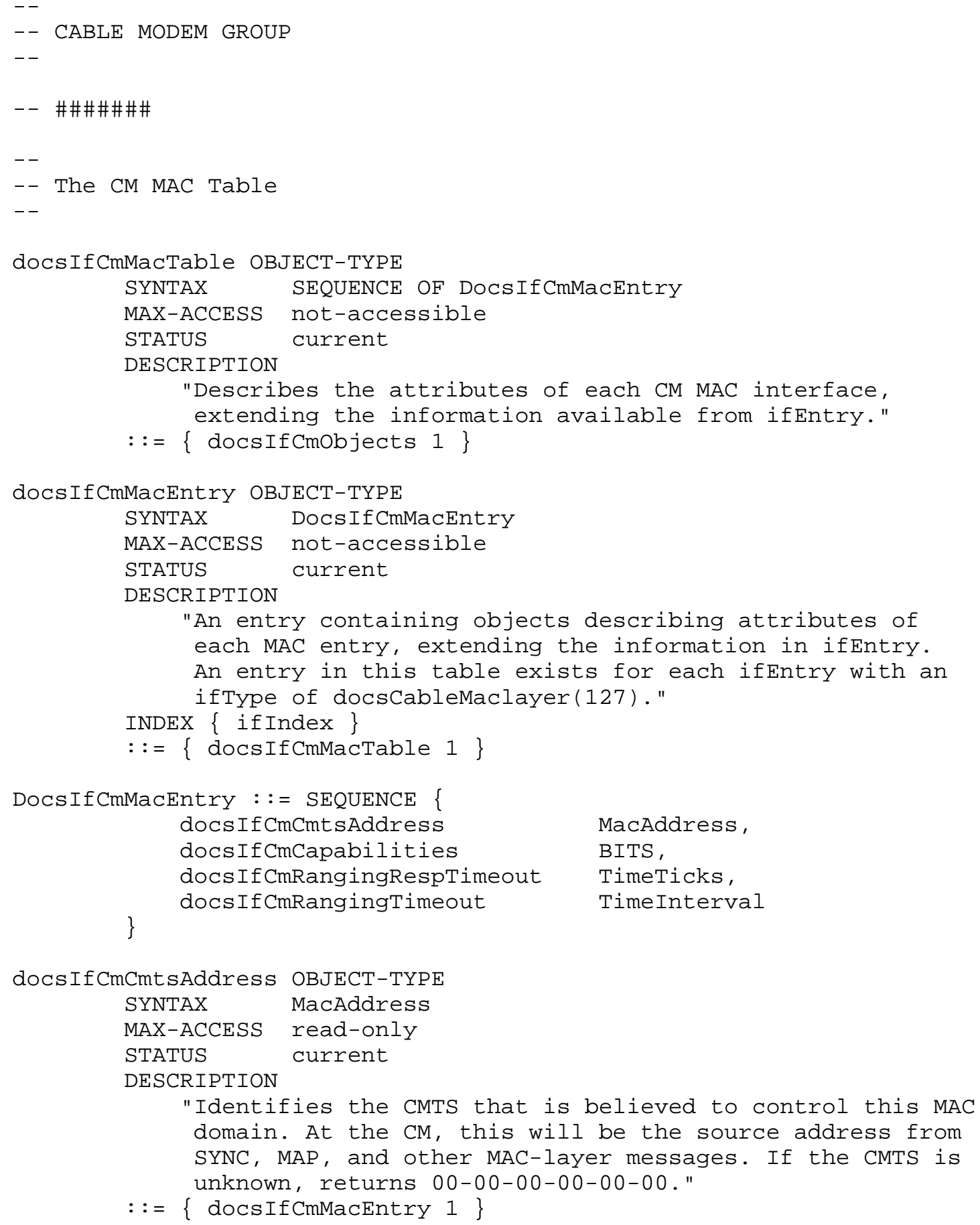




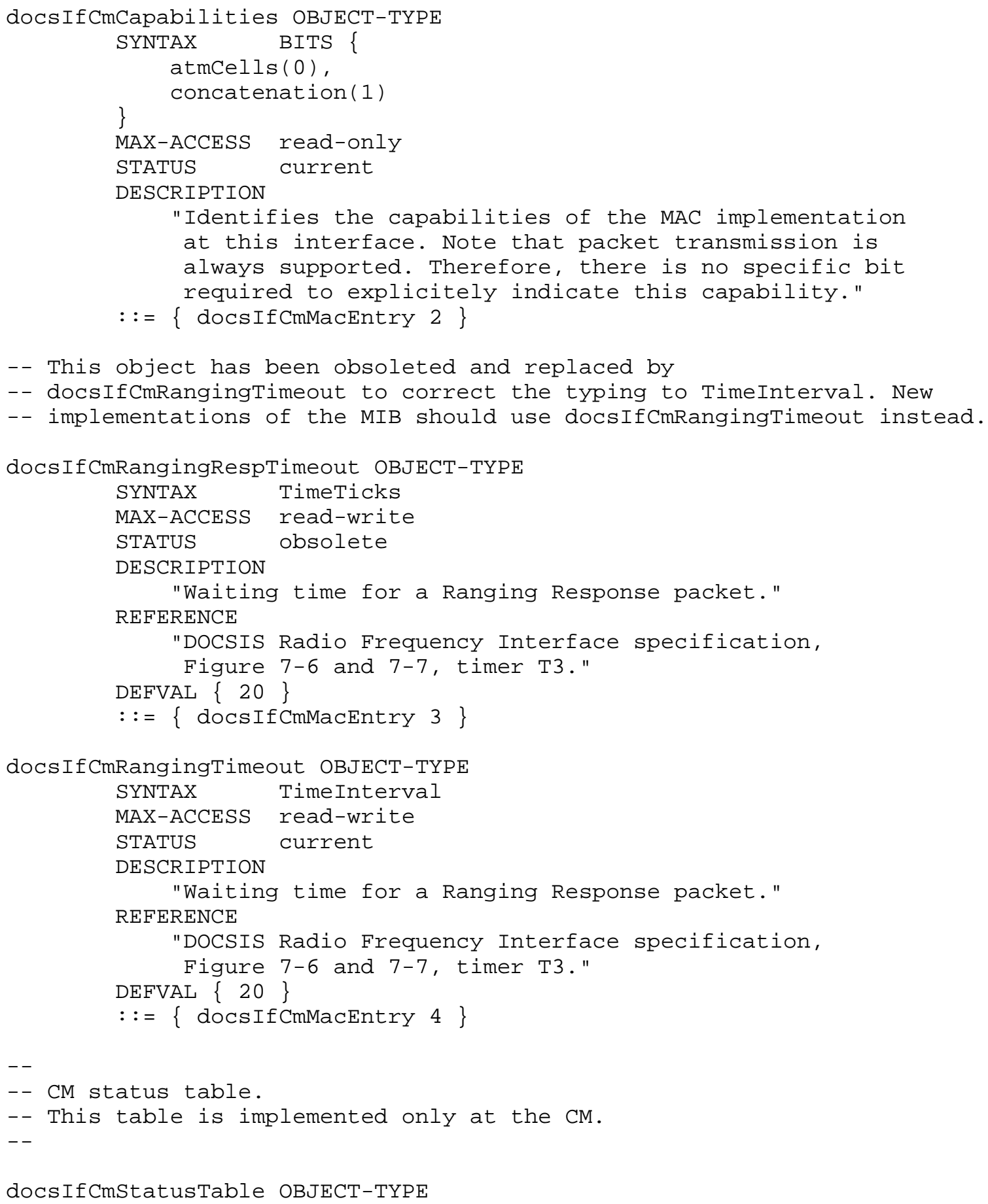




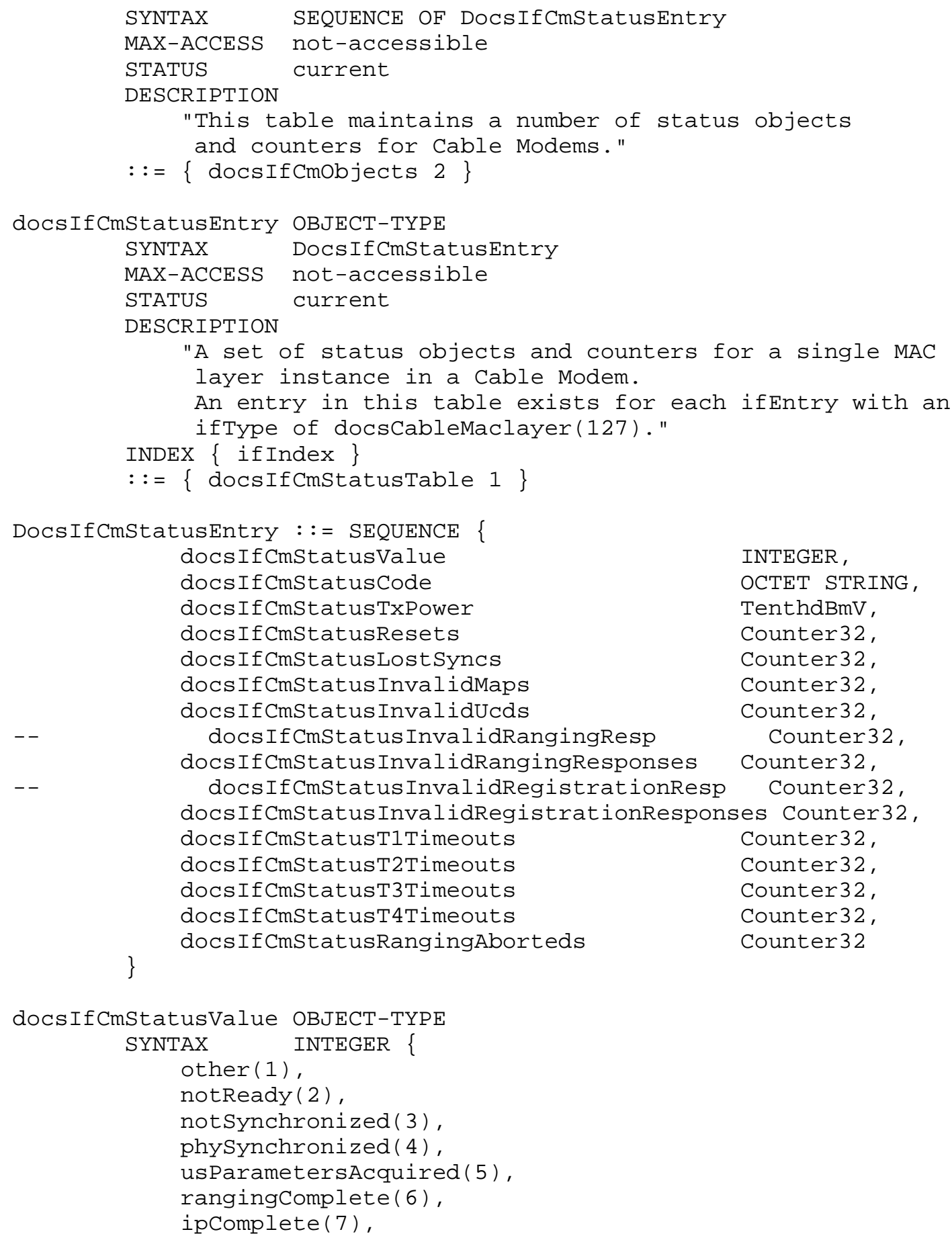




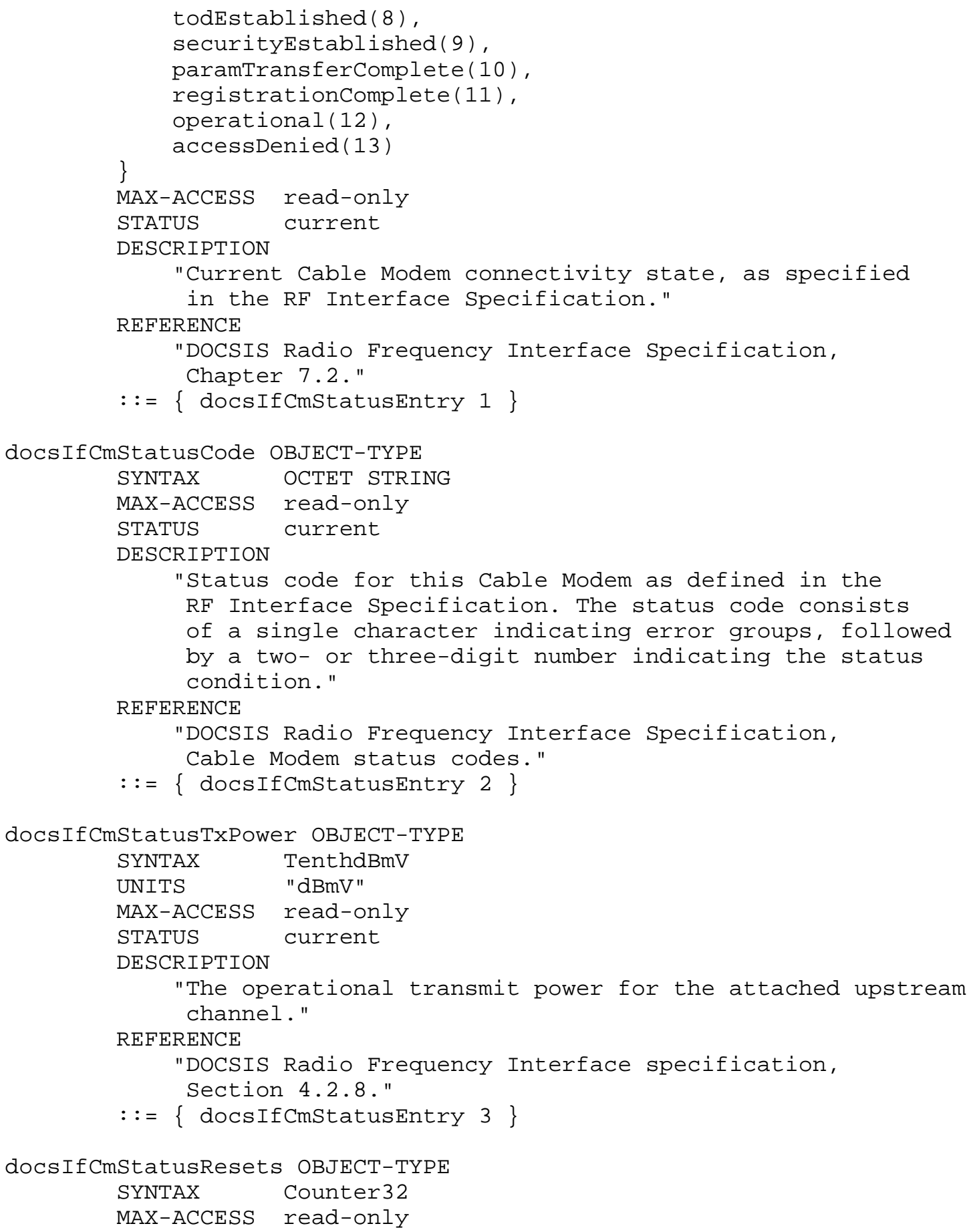




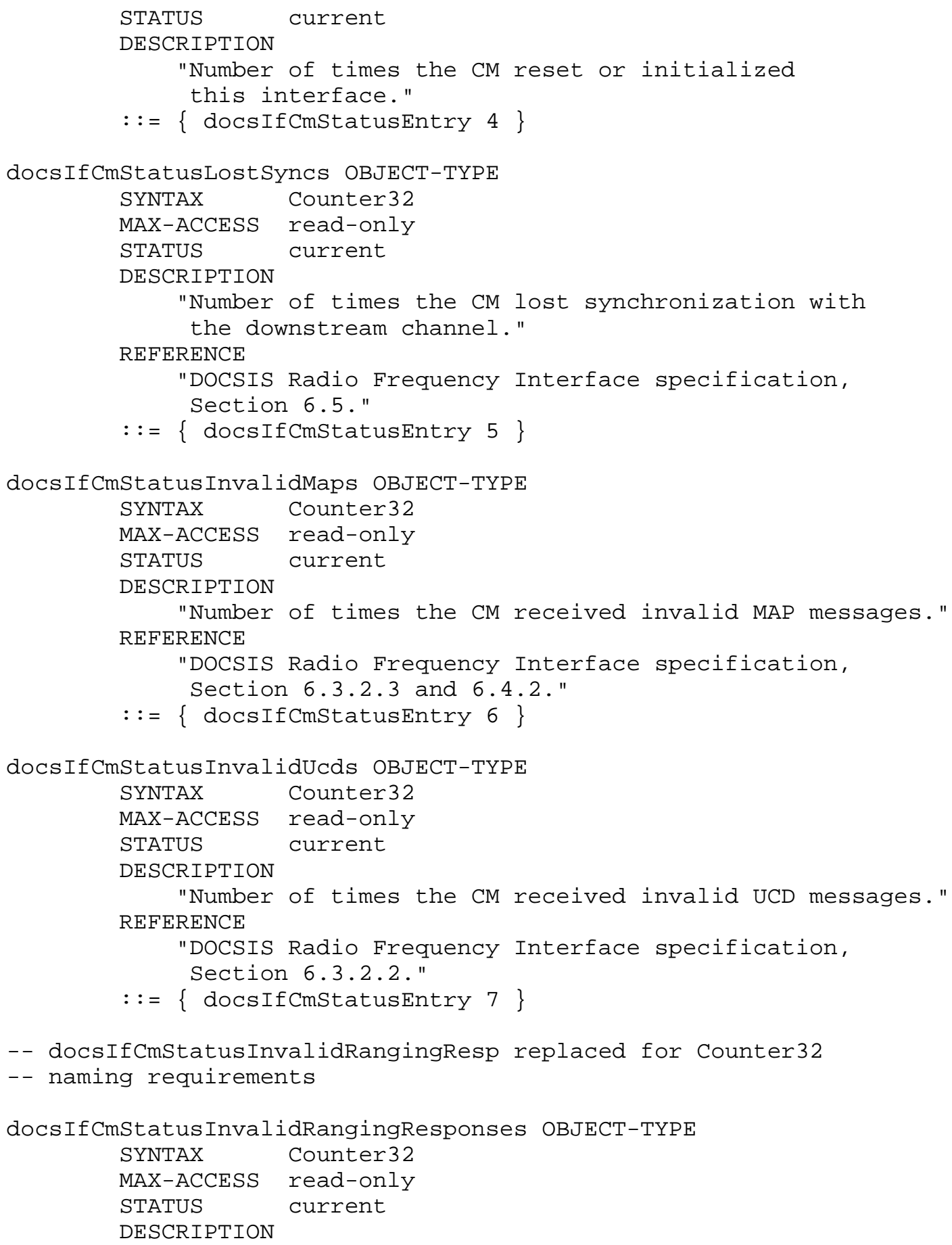




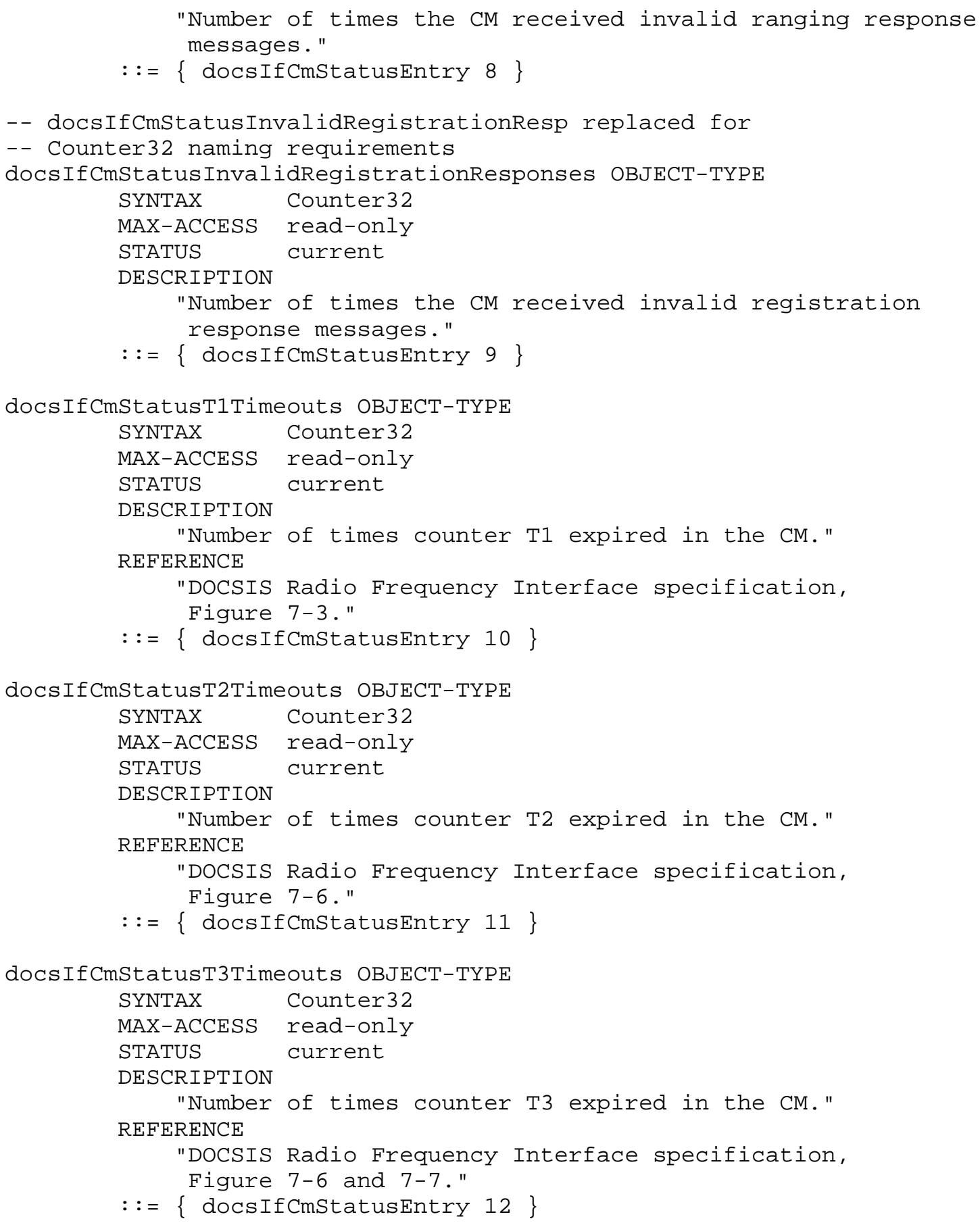




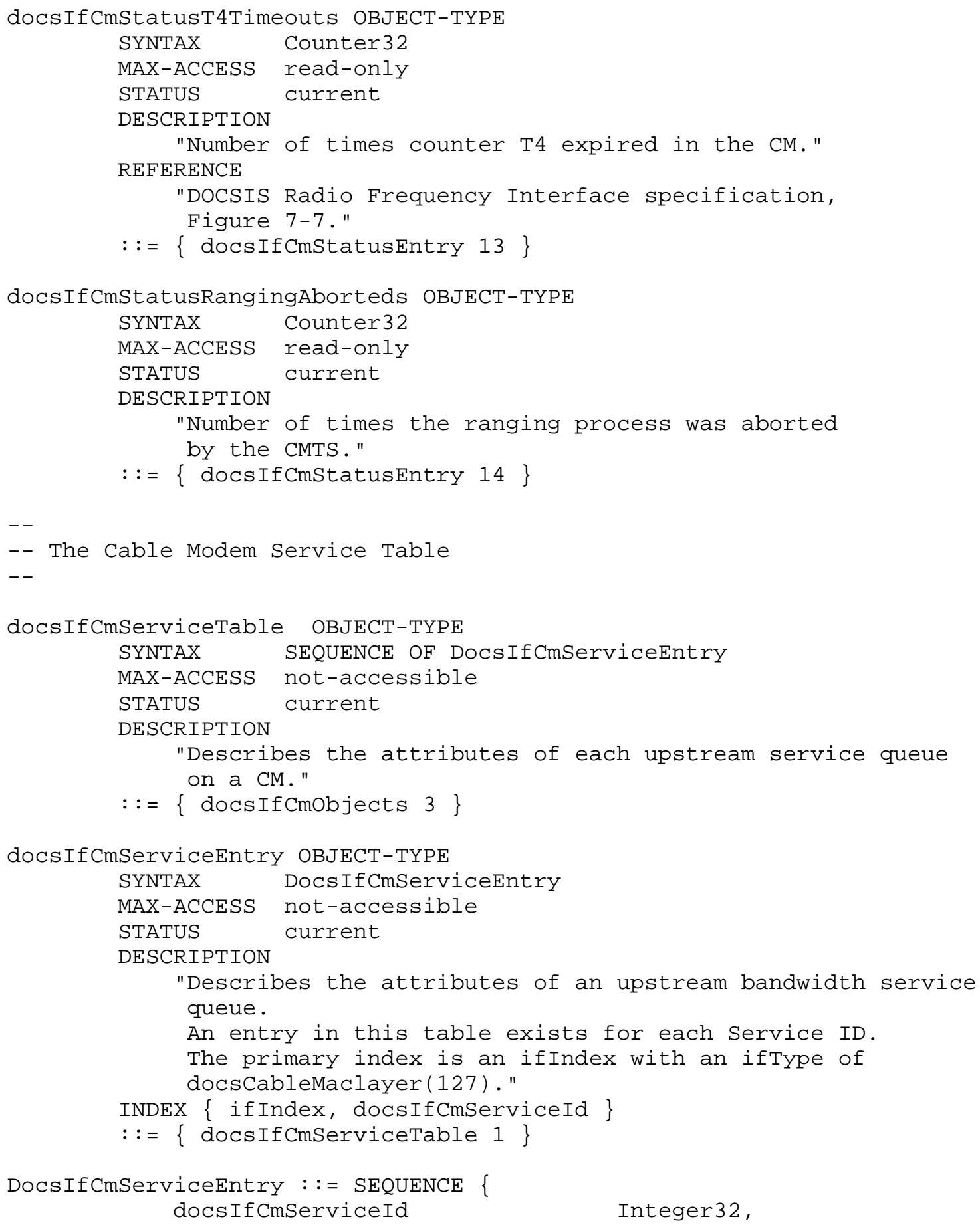




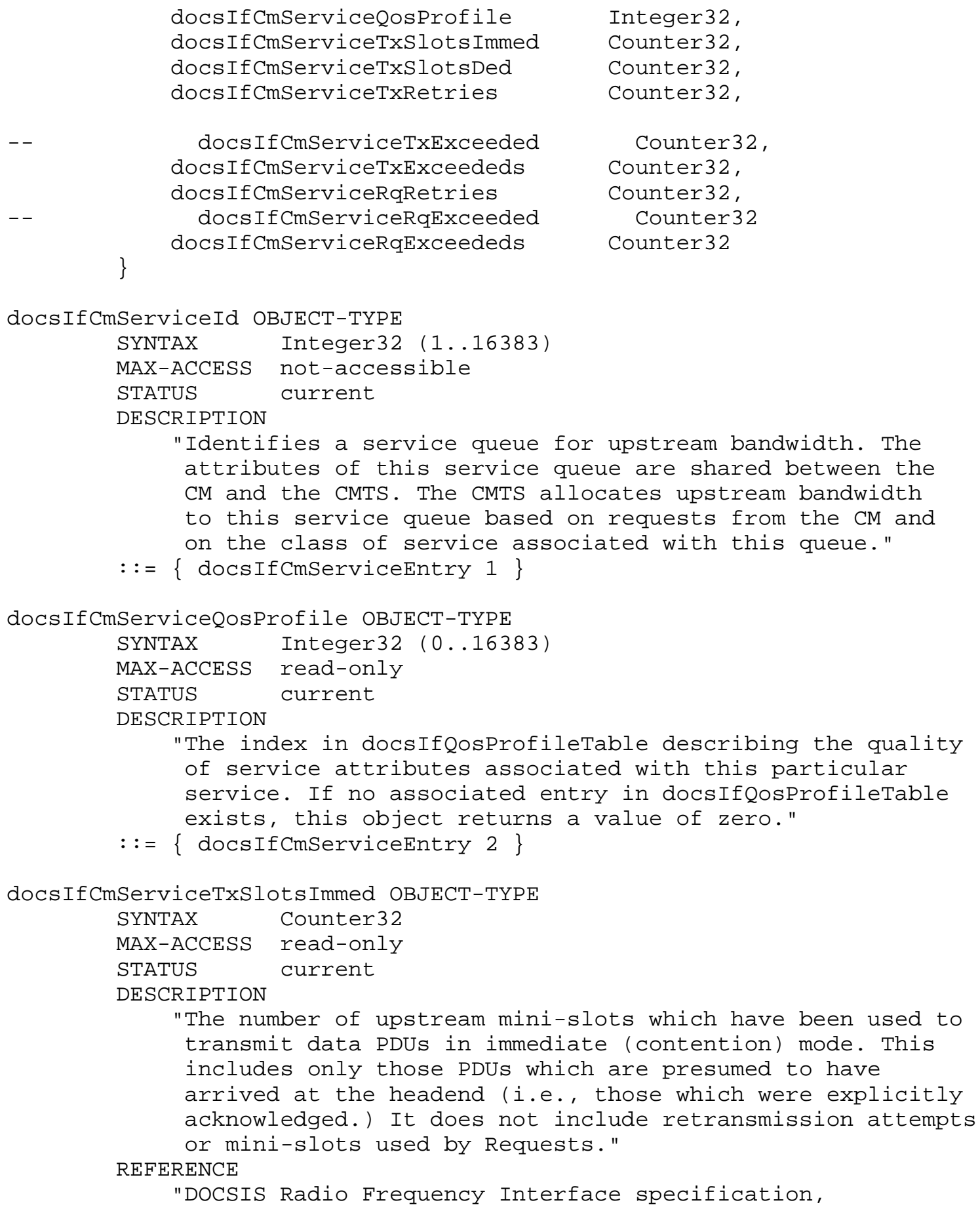




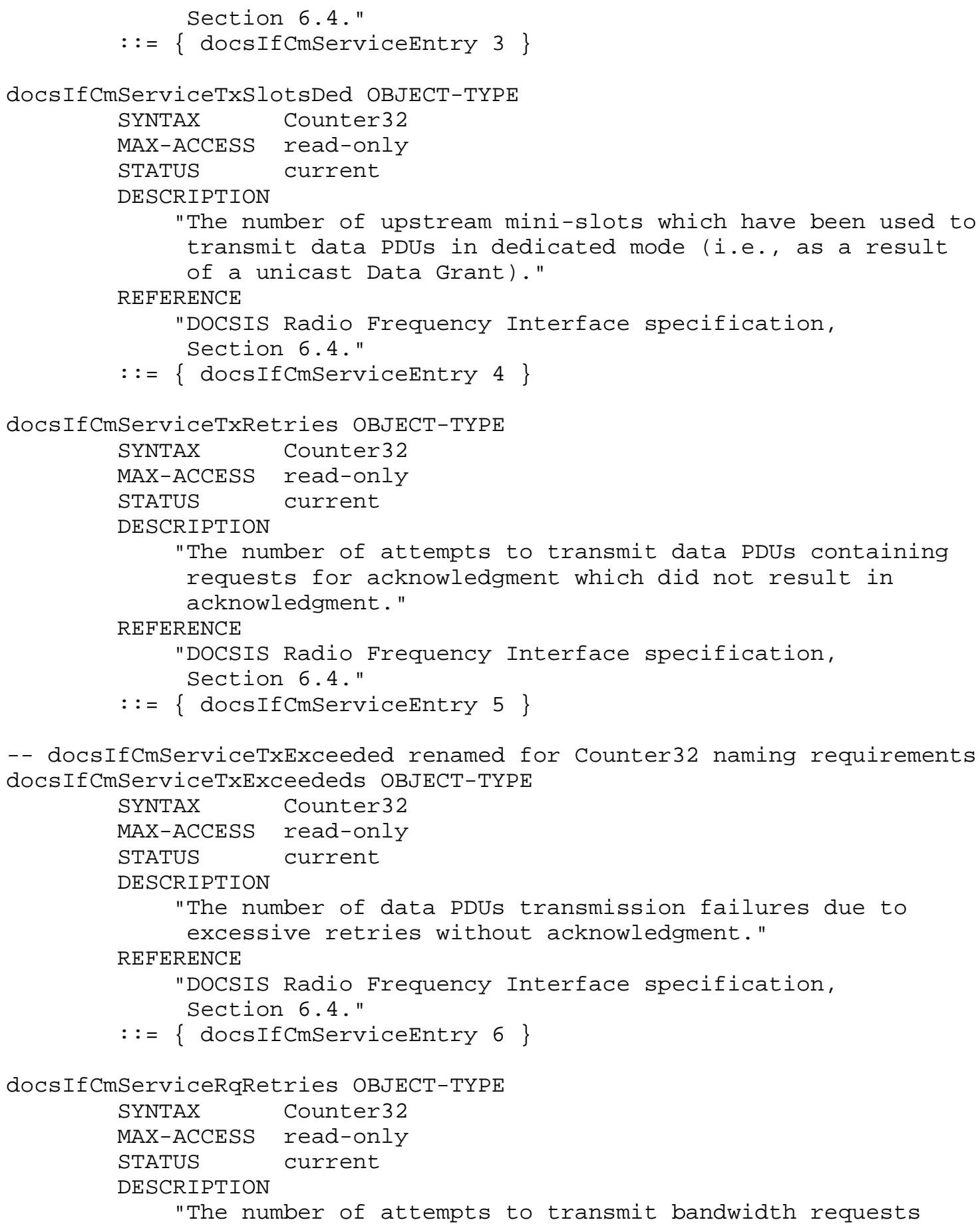




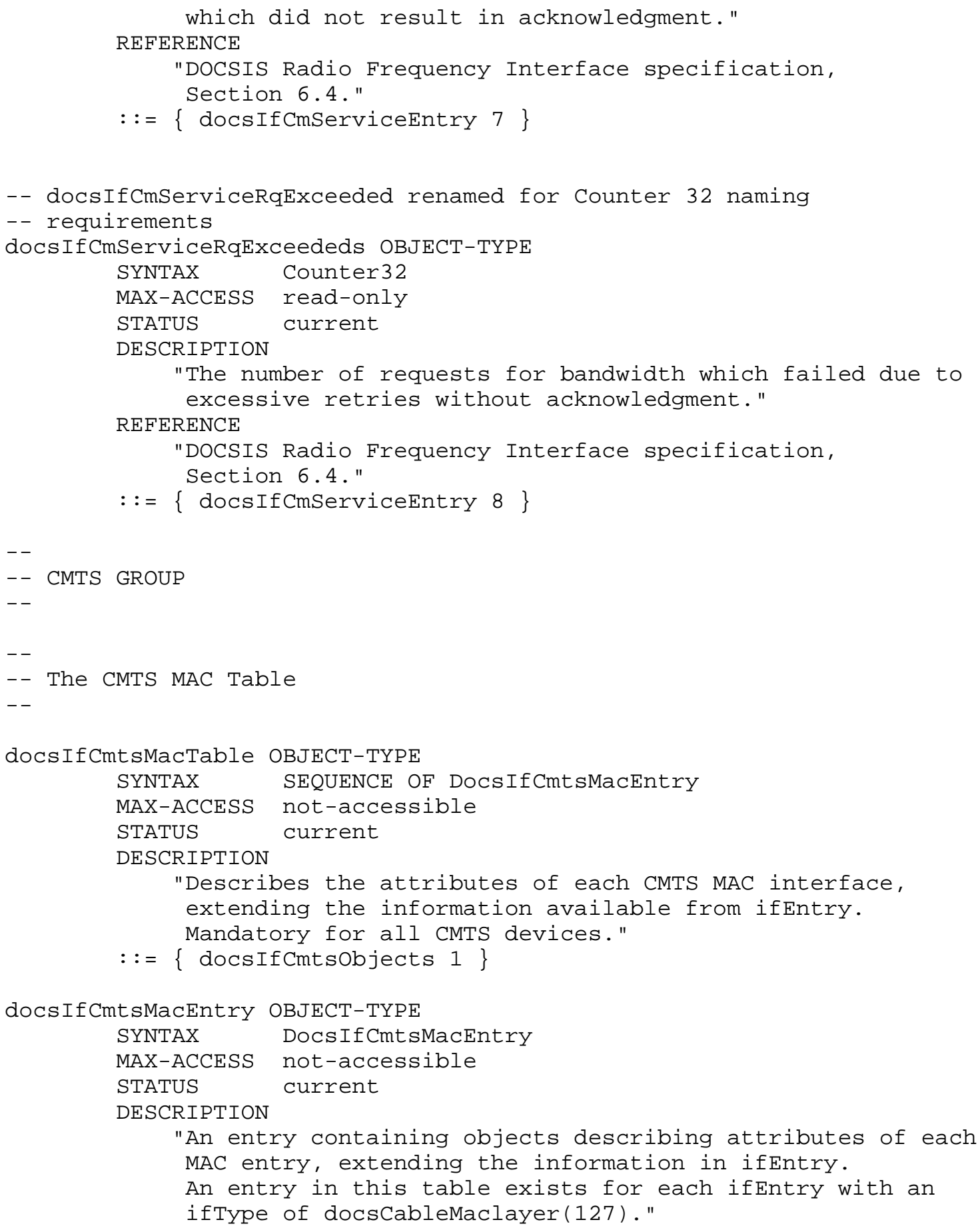


INDEX \{ ifIndex $\}$

$::=\{$ docsifcmtsmactable 1$\}$

DocsIfCmtsMacEntry : := SEQUENCE

docsifCmtsCapabilities BITS,

docsIfCmts SyncInterval Integer32,

docsifCmtsUcdInterval Integer32,

docsifCmtsMaxServiceIds Integer32,

docsIfCmtsInsertionInterval TimeTicks,

docsIfCmtsInvitedRangingAttempts Integer32,

\} docsIfCmtsInsertInterval TimeInterval

docsIfCmtsCapabilities OBJECT-TYPE

SYNTAX BITS \{

atmCells $(0)$,

concatenation (1)

\}

MAX-ACCESS read-only

STATUS current

DESCRIPTION

"Identifies the capabilities of the CMTS MAC

implementation at this interface. Note that packet transmission is always supported. Therefore, there is no specific bit required to explicitely indicate this capability."

REFERENCE

"DOCSIS Radio Frequency Interface specification, Chapter 6."

$::=\{$ docsifCmtsMacEntry 1$\}$

docsIfCmts SyncInterval OBJECT-TYPE

SYNTAX Integer32 (1..200)

UNITS "Milliseconds"

MAX-ACCESS read-write

STATUS current

DESCRIPTION

"The interval between CMTS transmission of successive SYNC messages at this interface."

REFERENCE

"DOCSIS Radio Frequency Interface Specification, Section 6.5 and Appendix B."

$::=\{$ docsifCmtsMacEntry 2$\}$

docsIfCmtsUcdInterval OBJECT-TYPE

SYNTAX Integer32 (1..2000)

UNITS "Miliseconds"

MAX-ACCESS read-write 


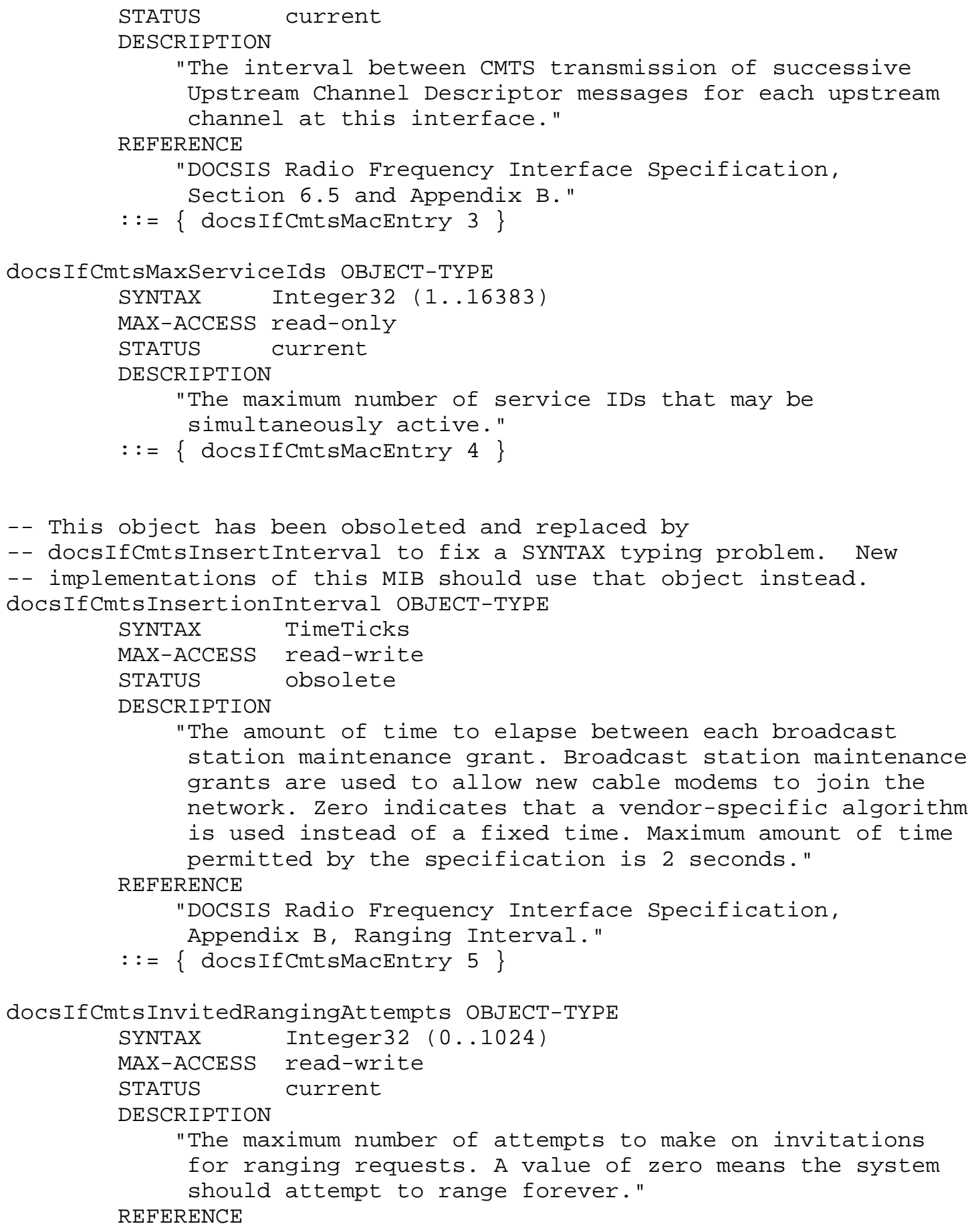




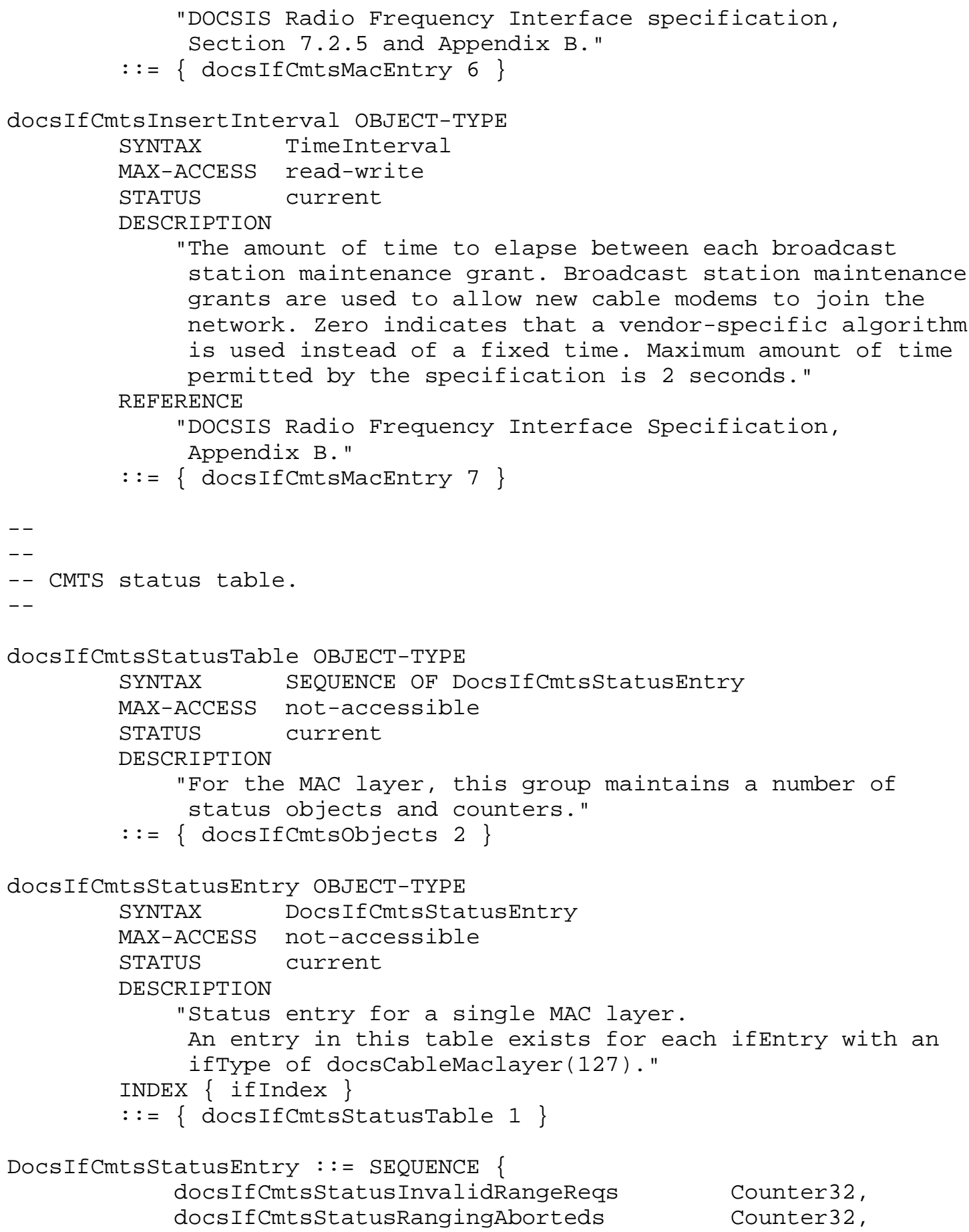




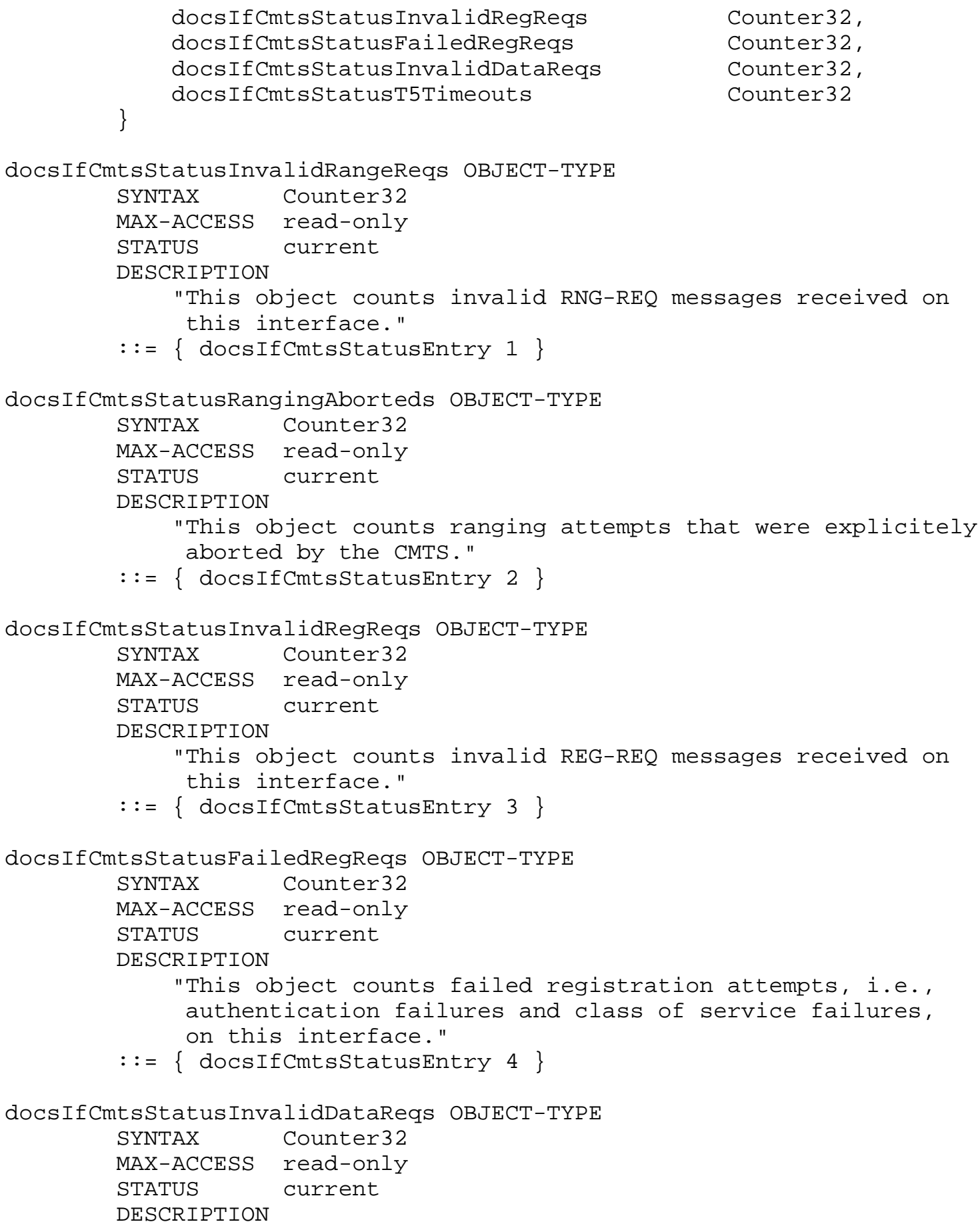




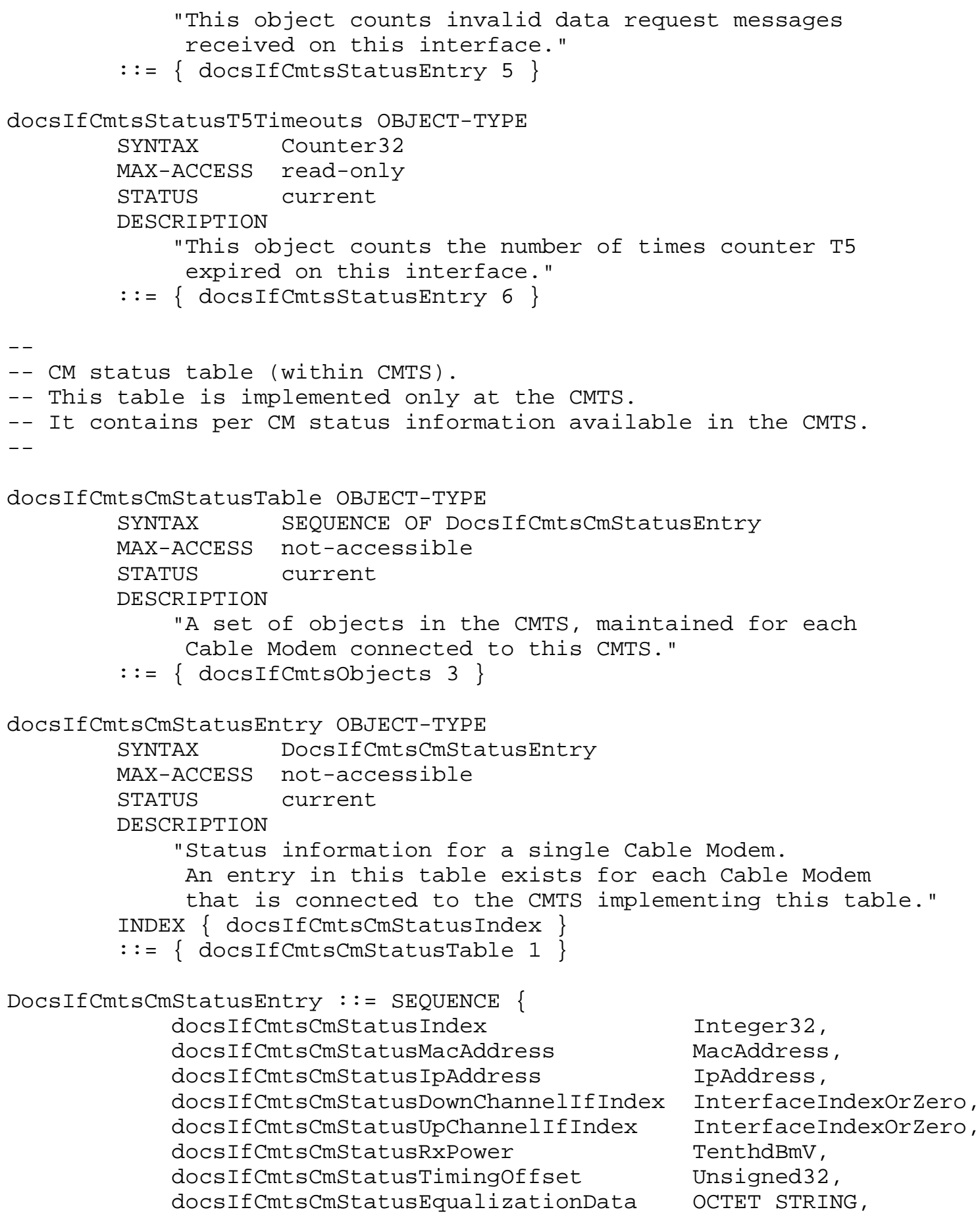




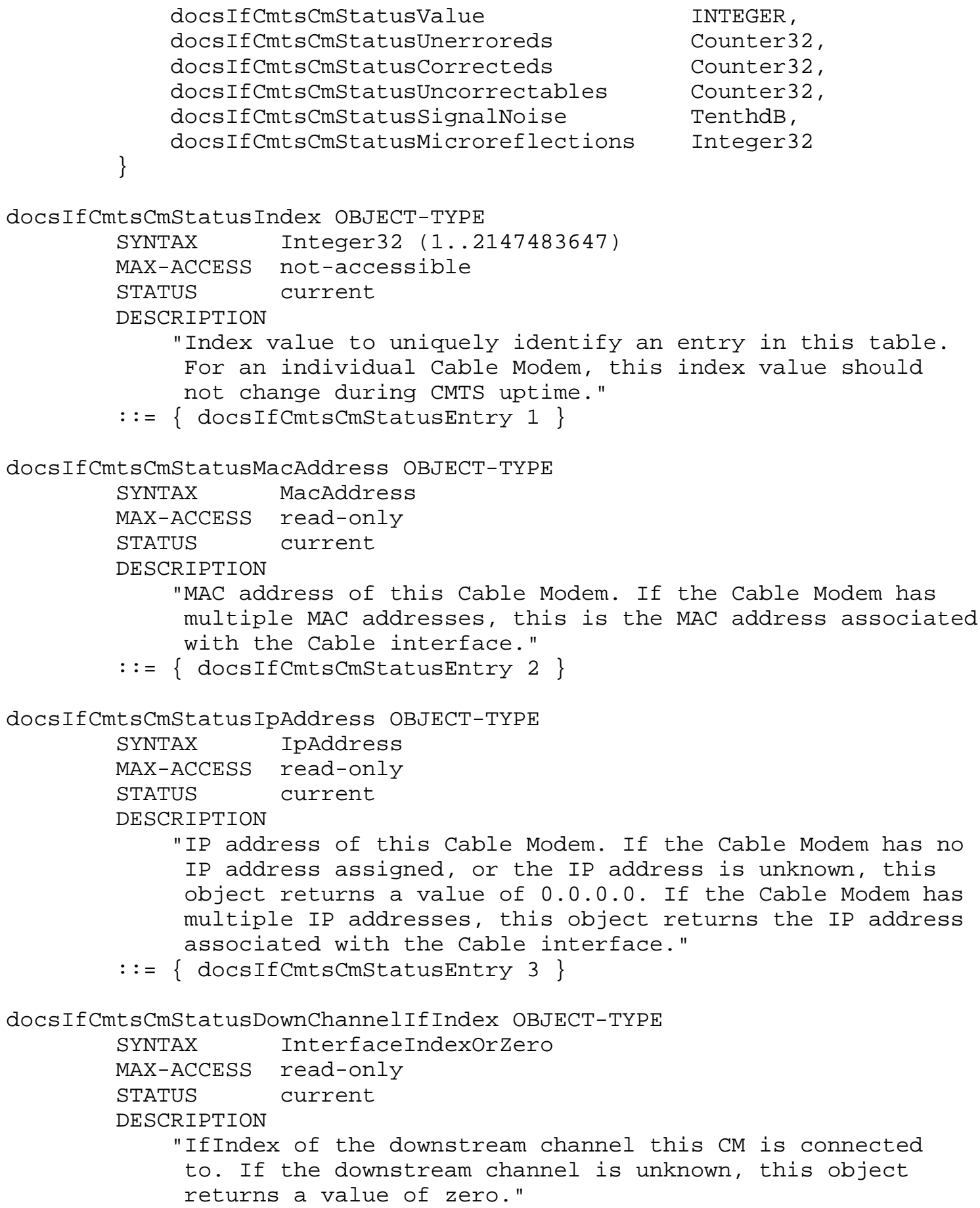




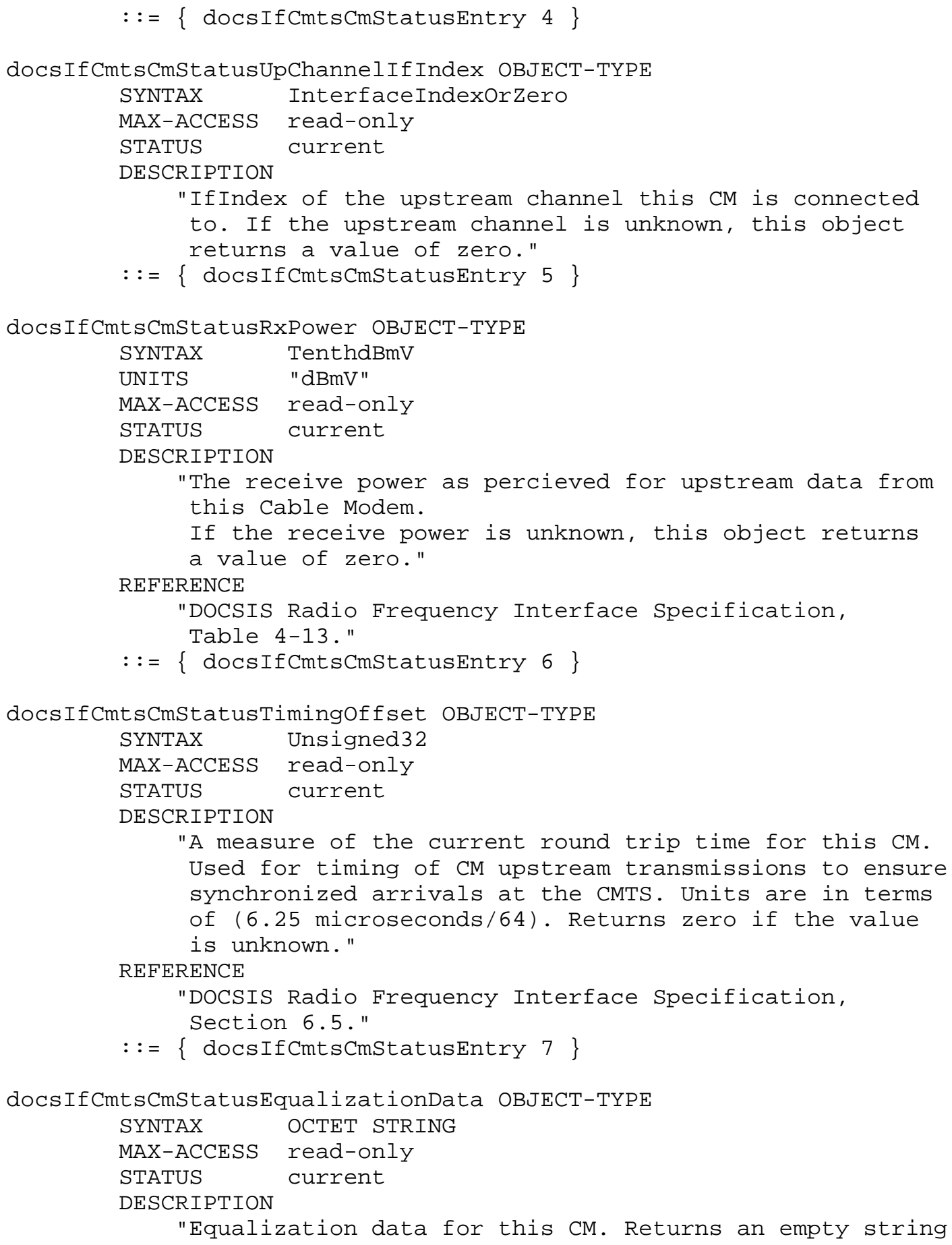




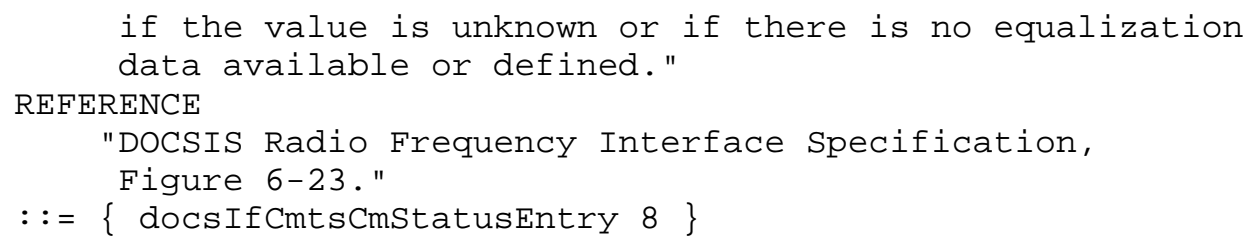




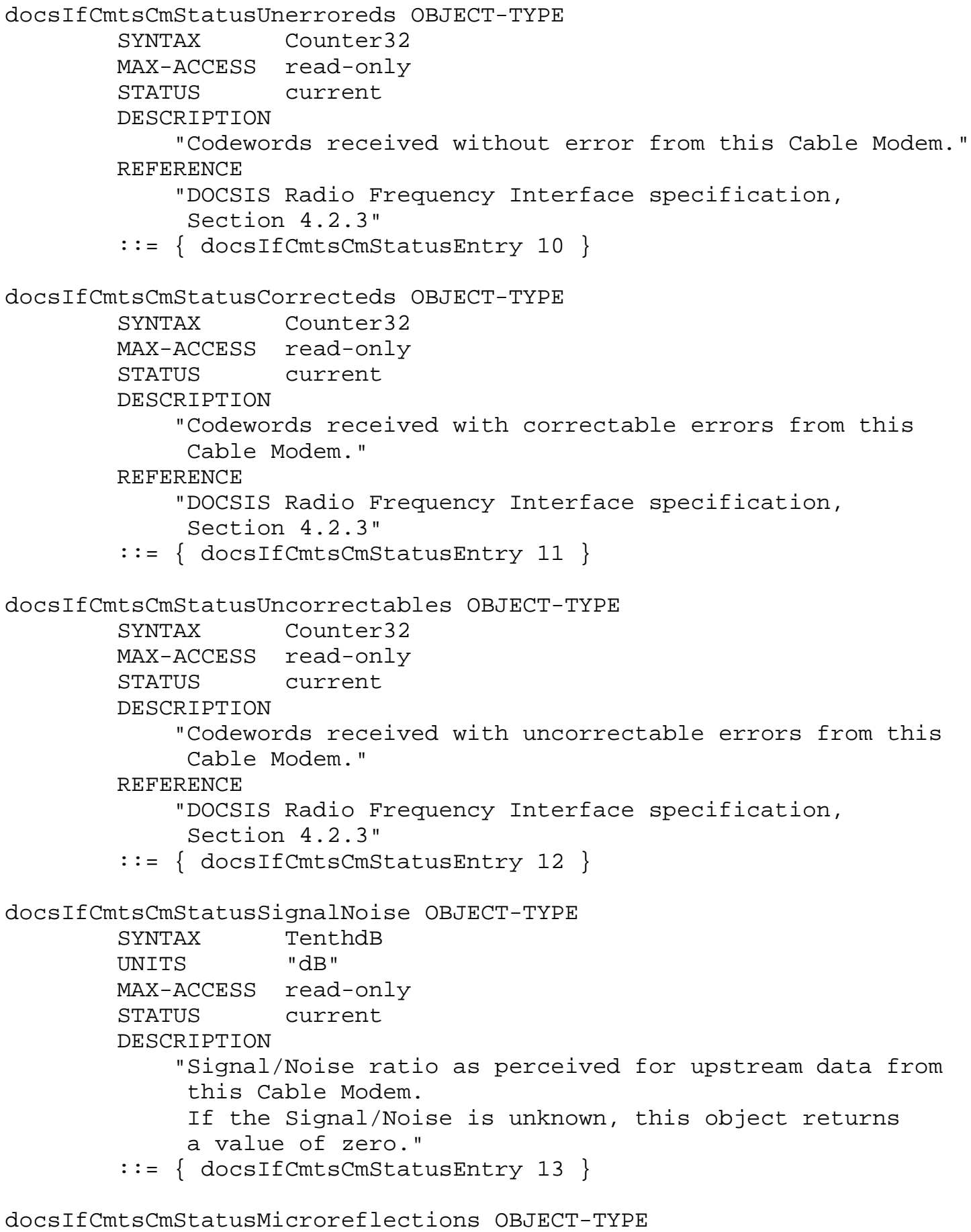




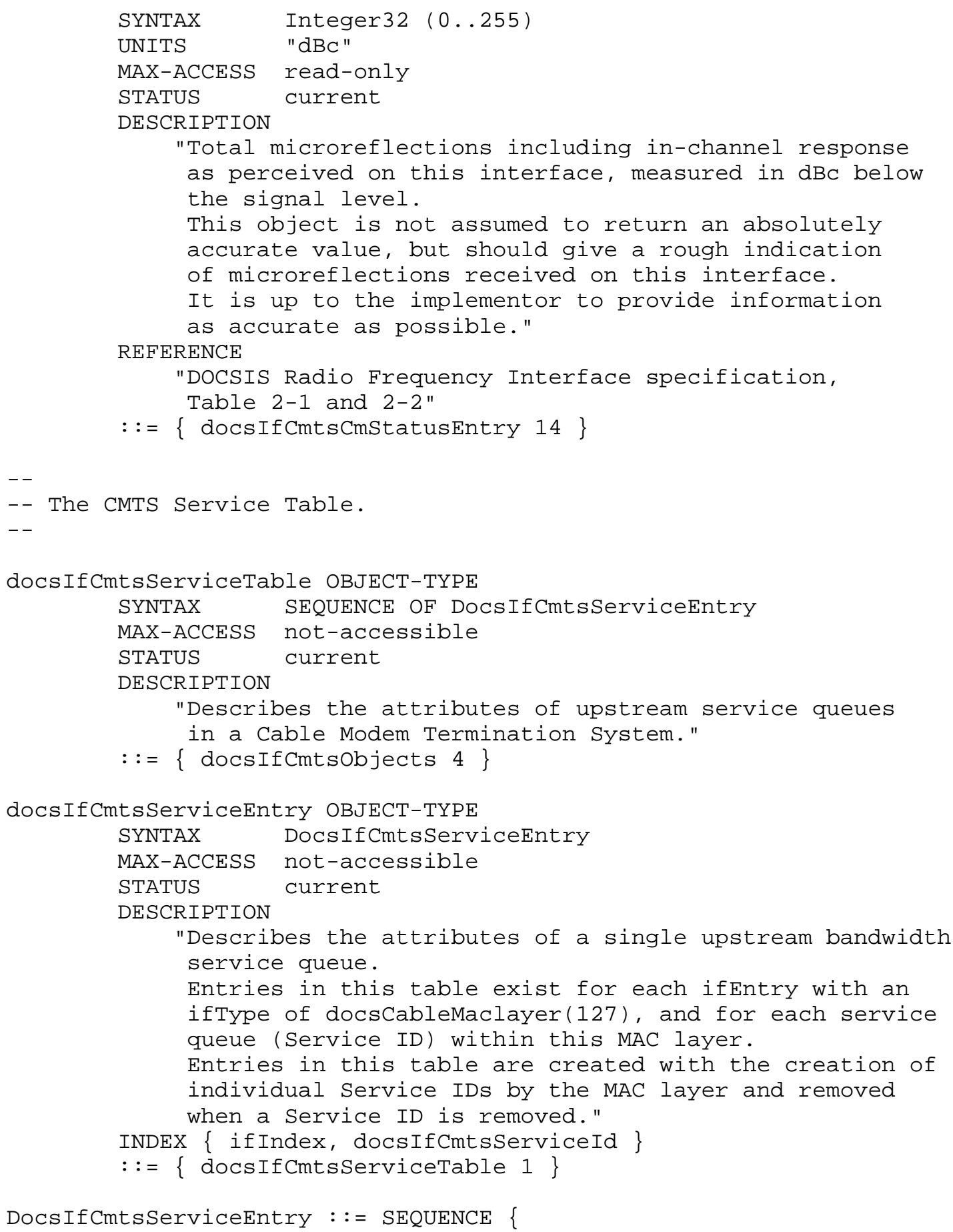




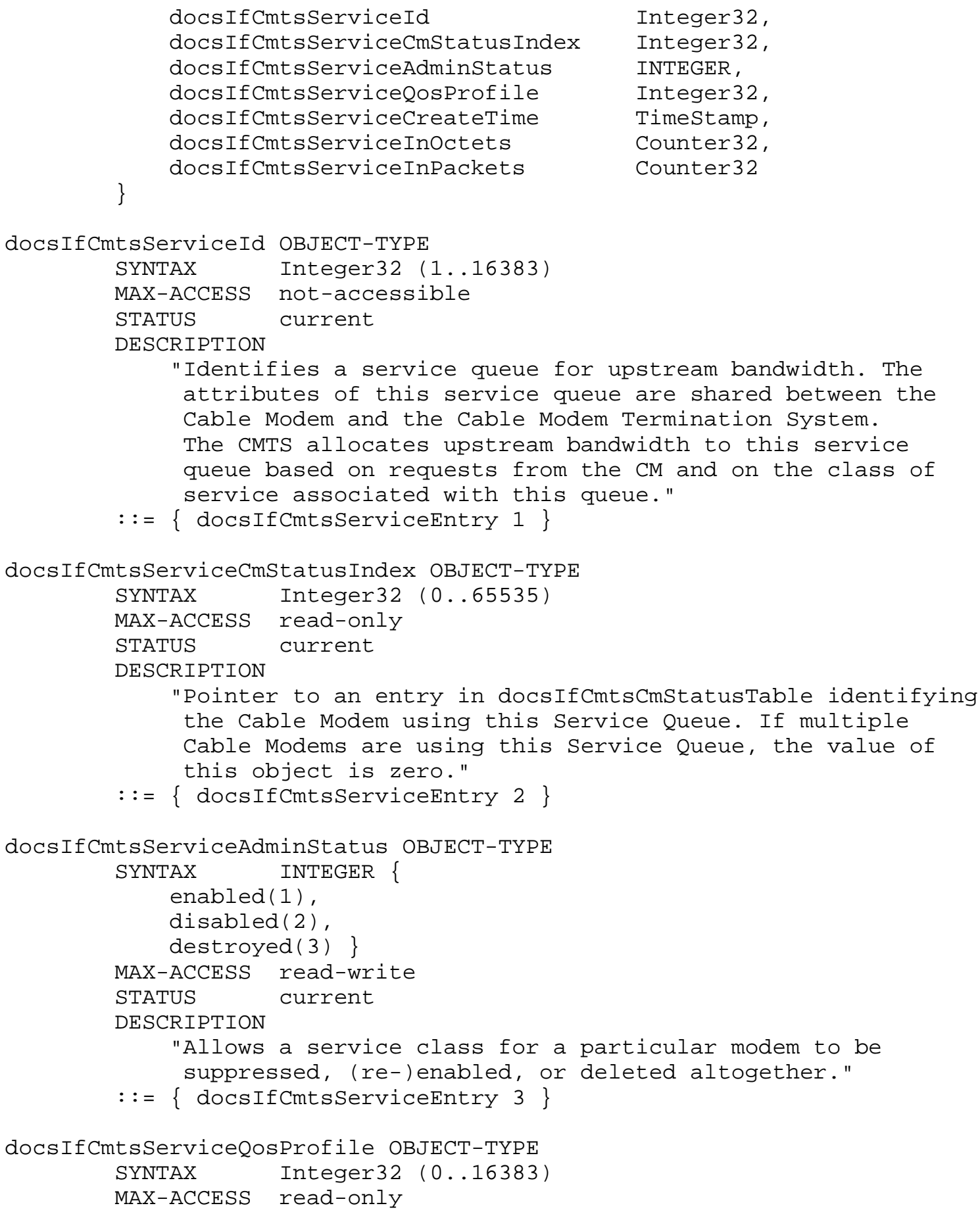




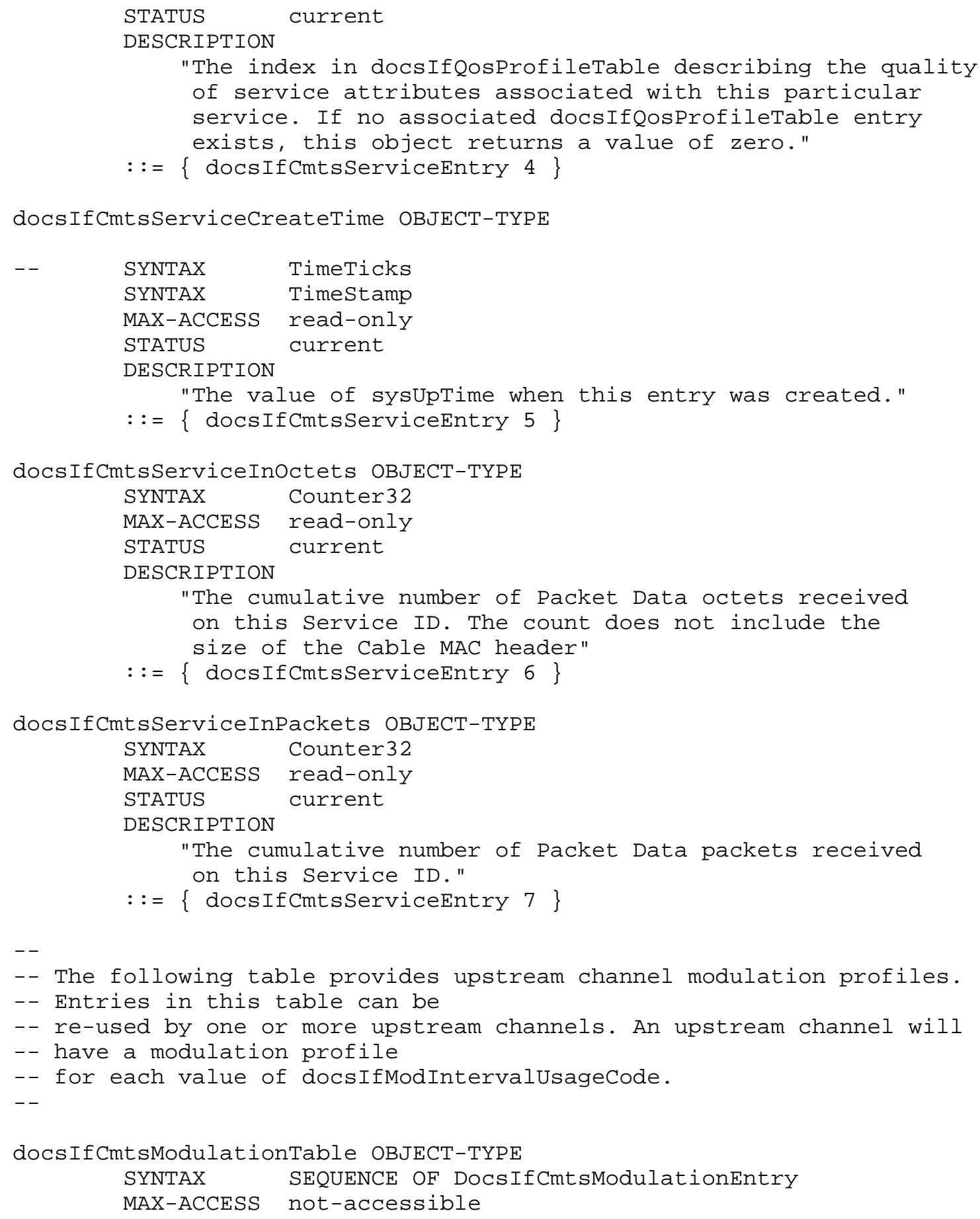




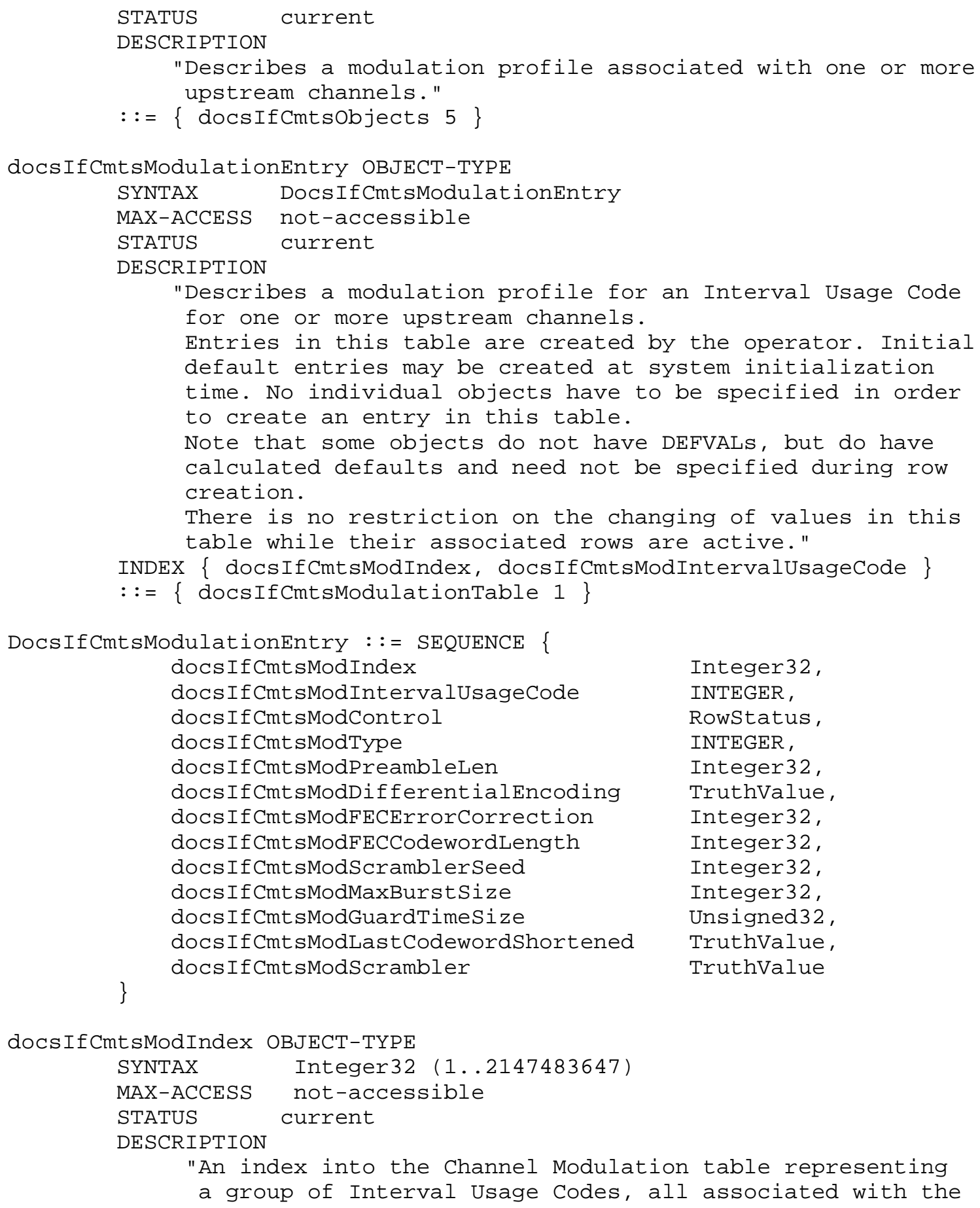




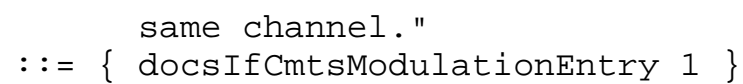




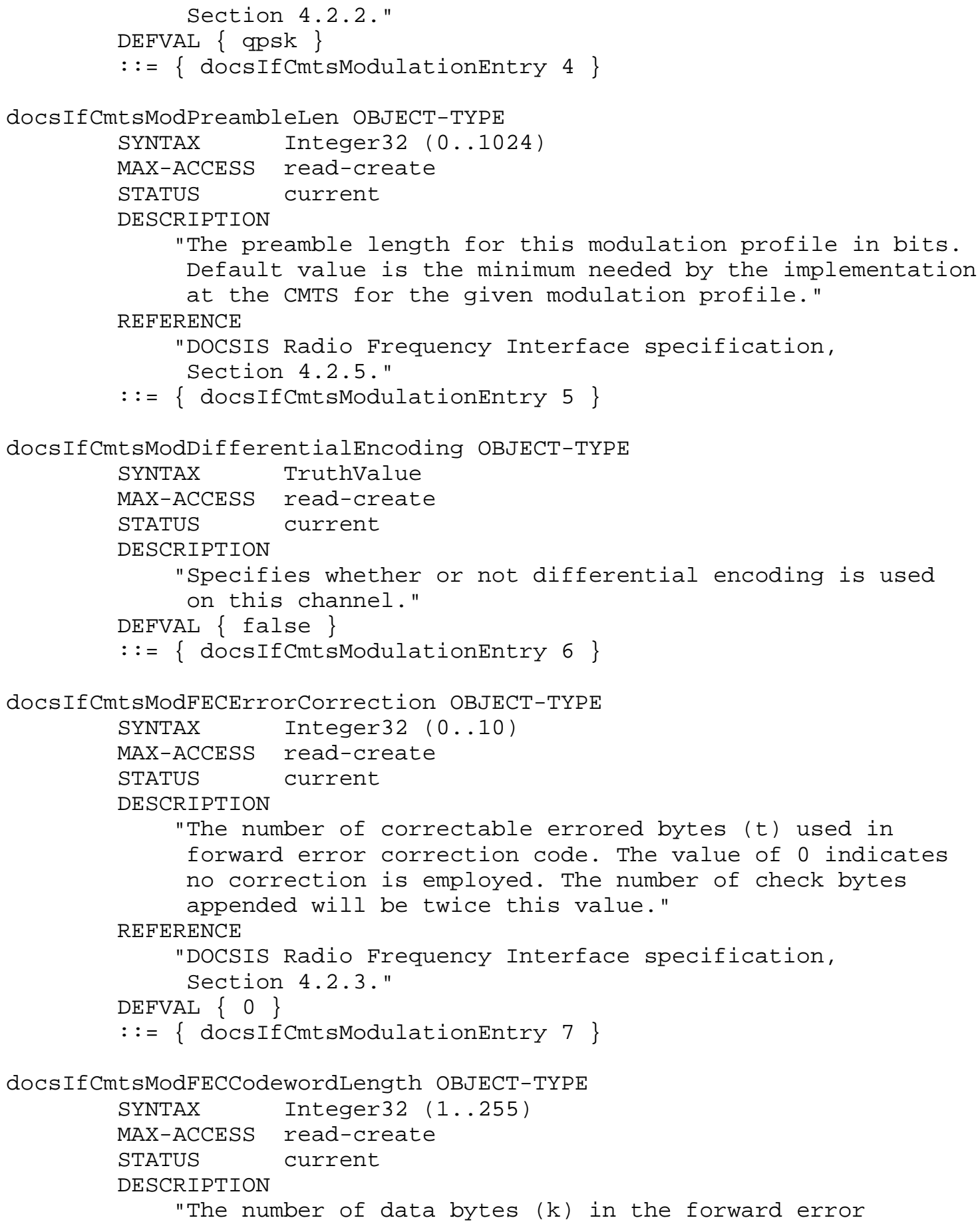




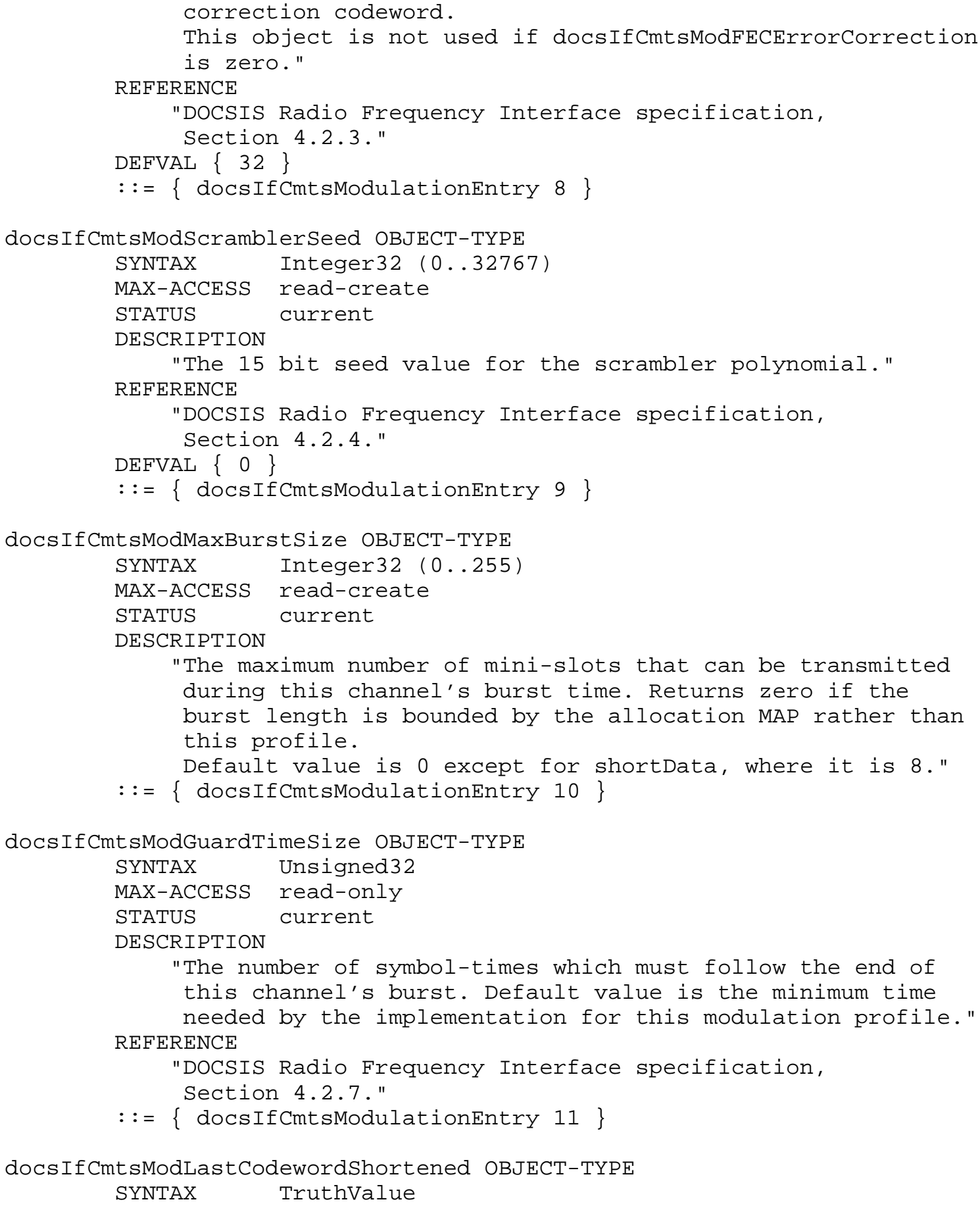




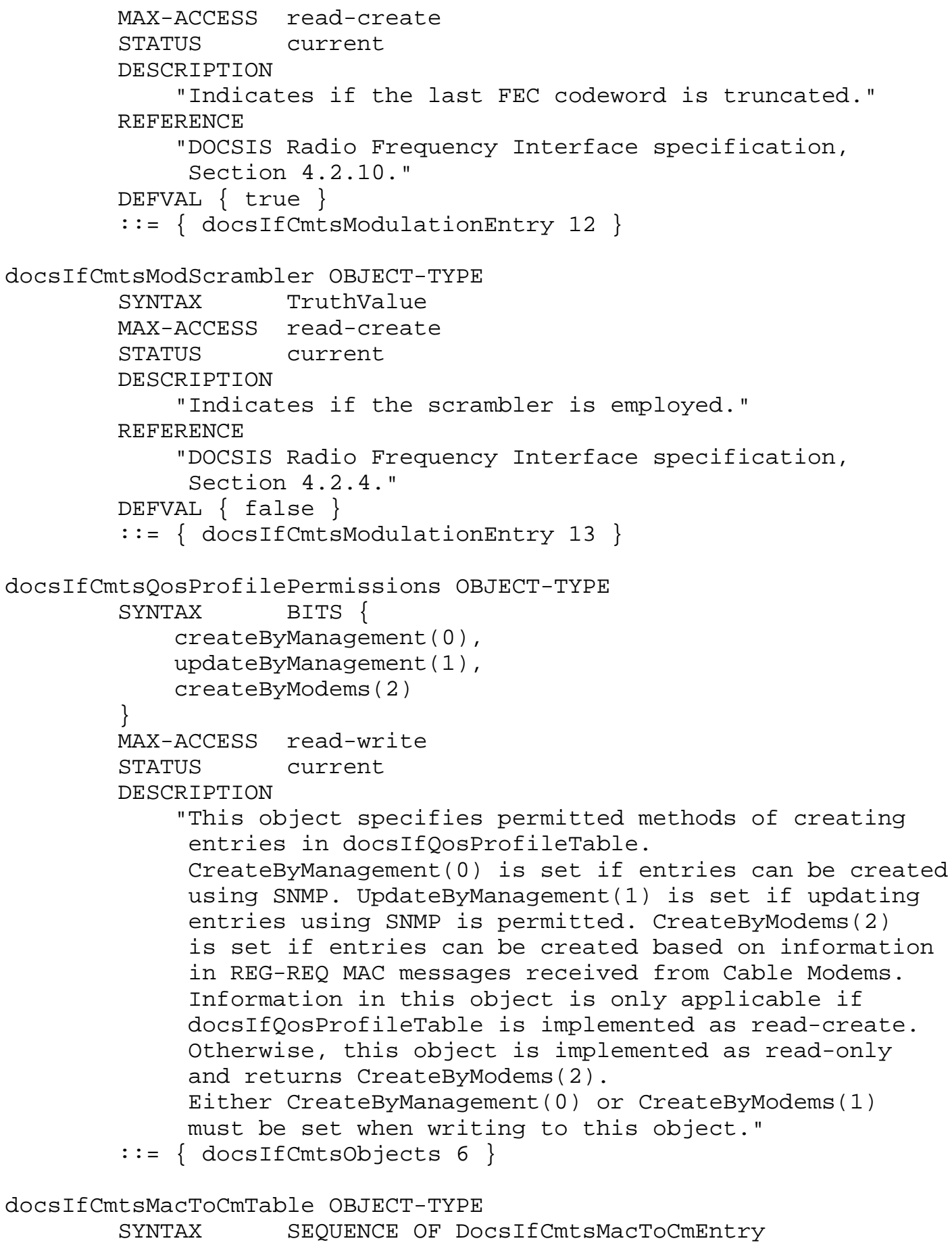




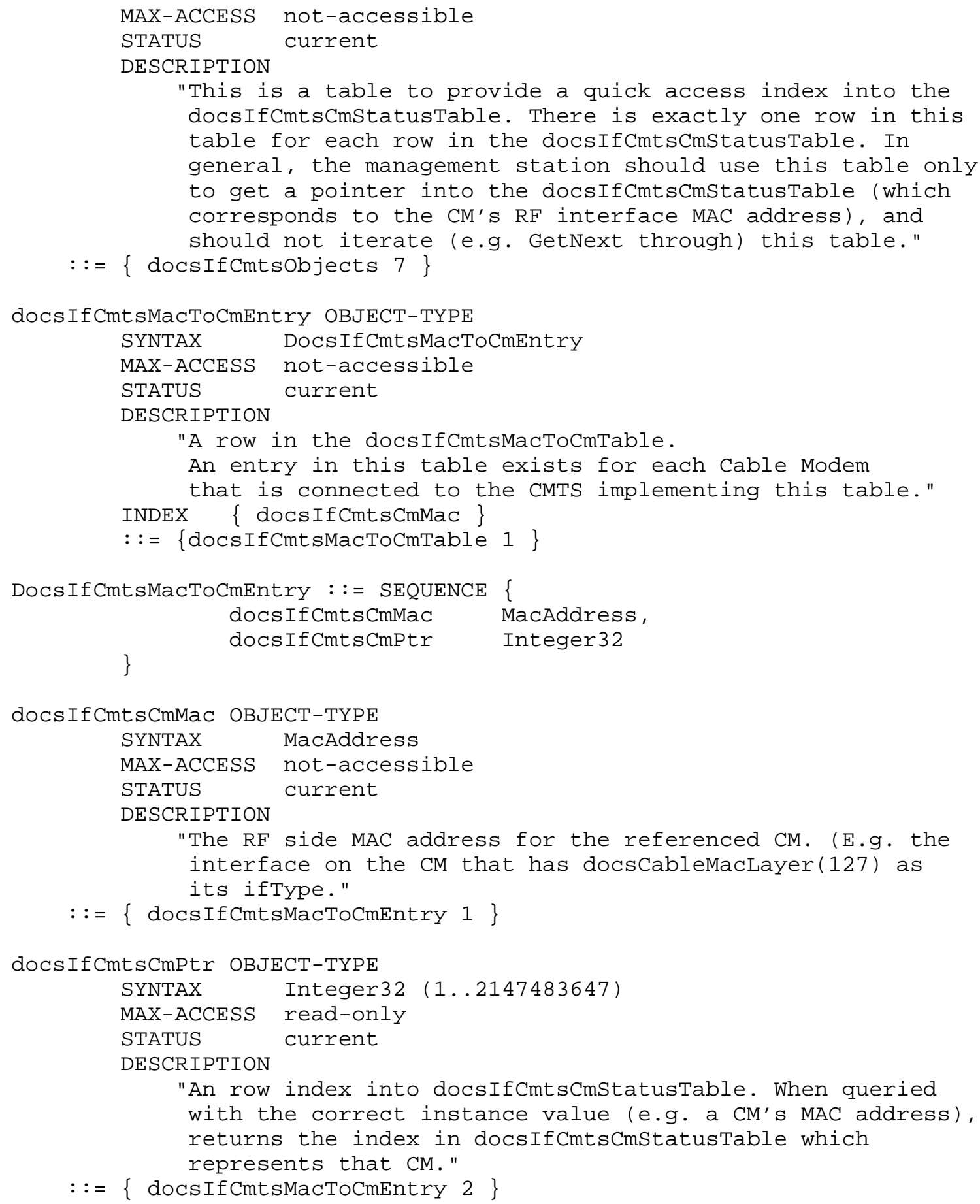




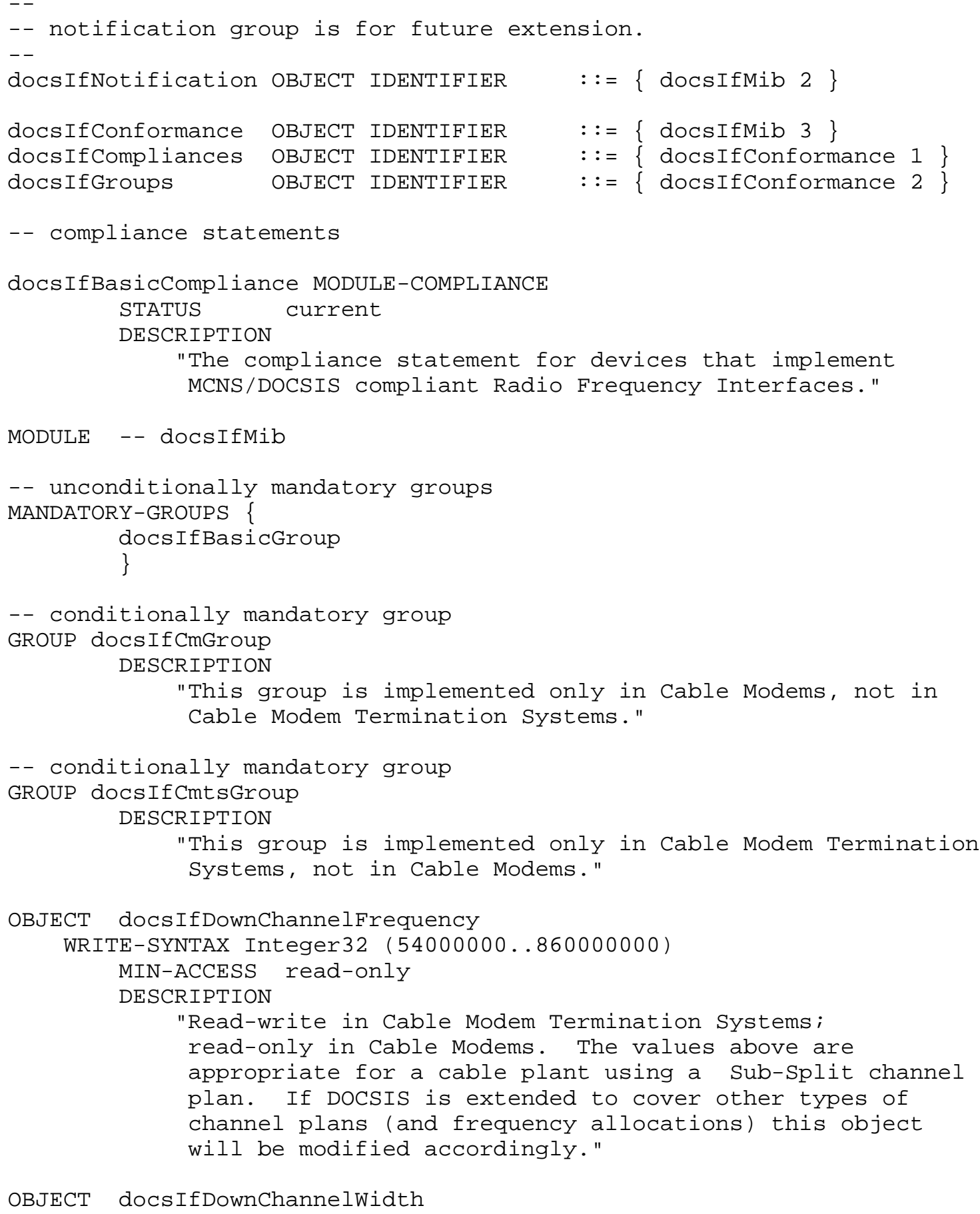




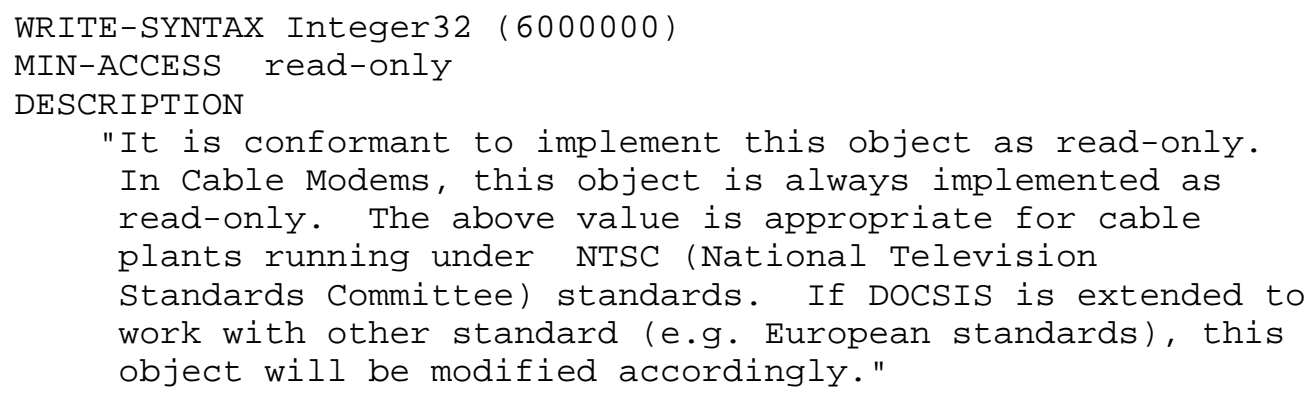




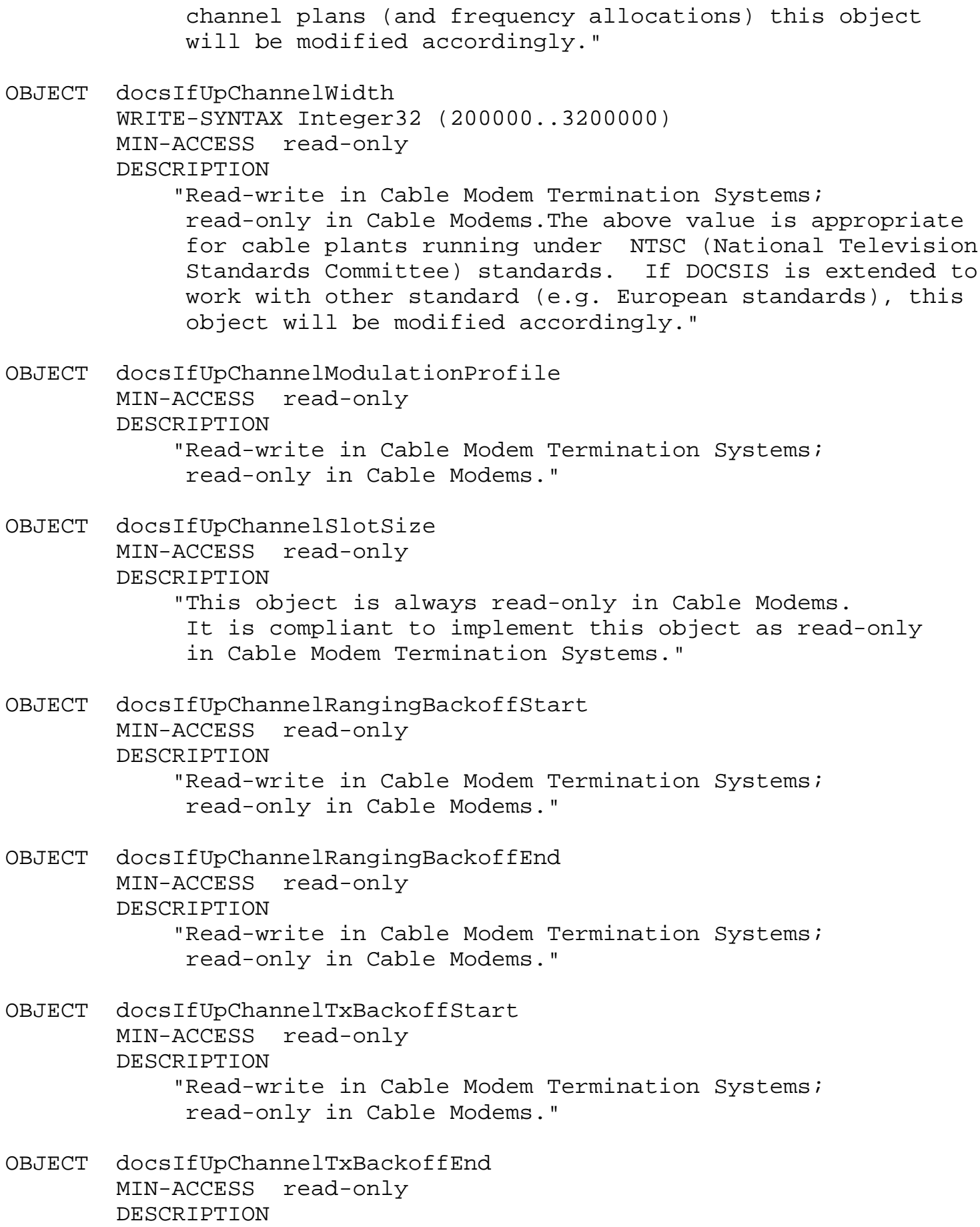




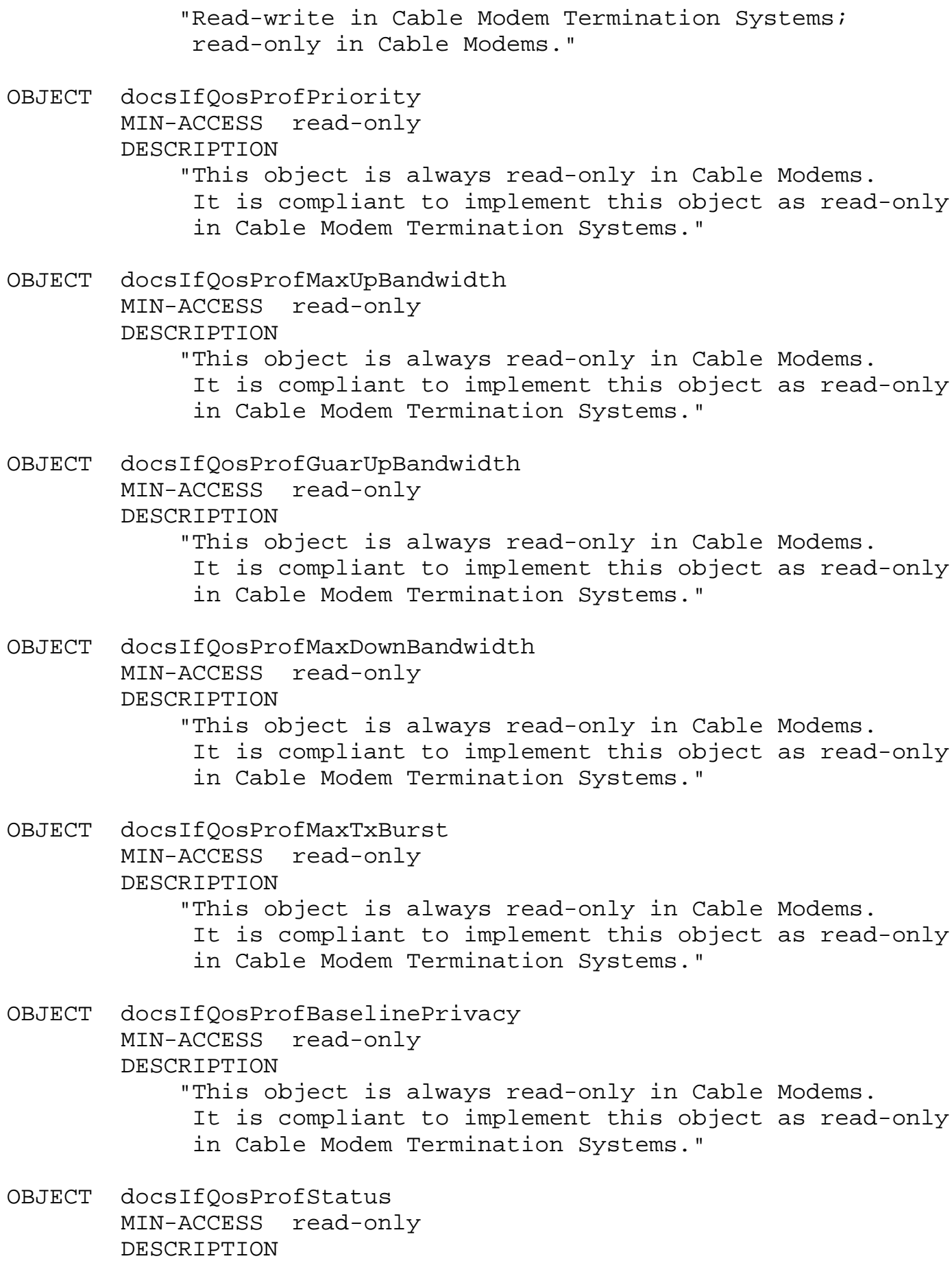




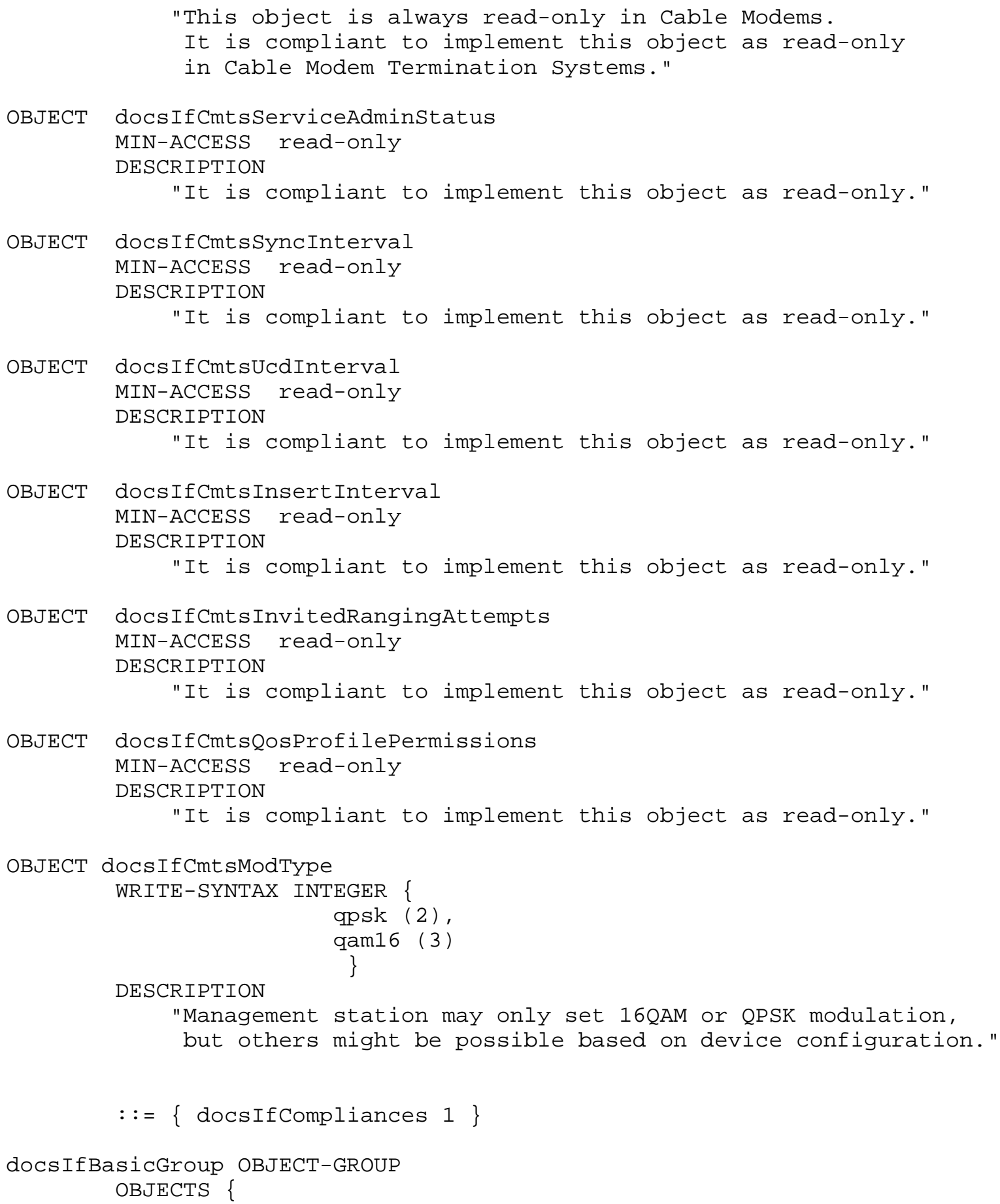

St. Johns

Standard

[Page 65] 


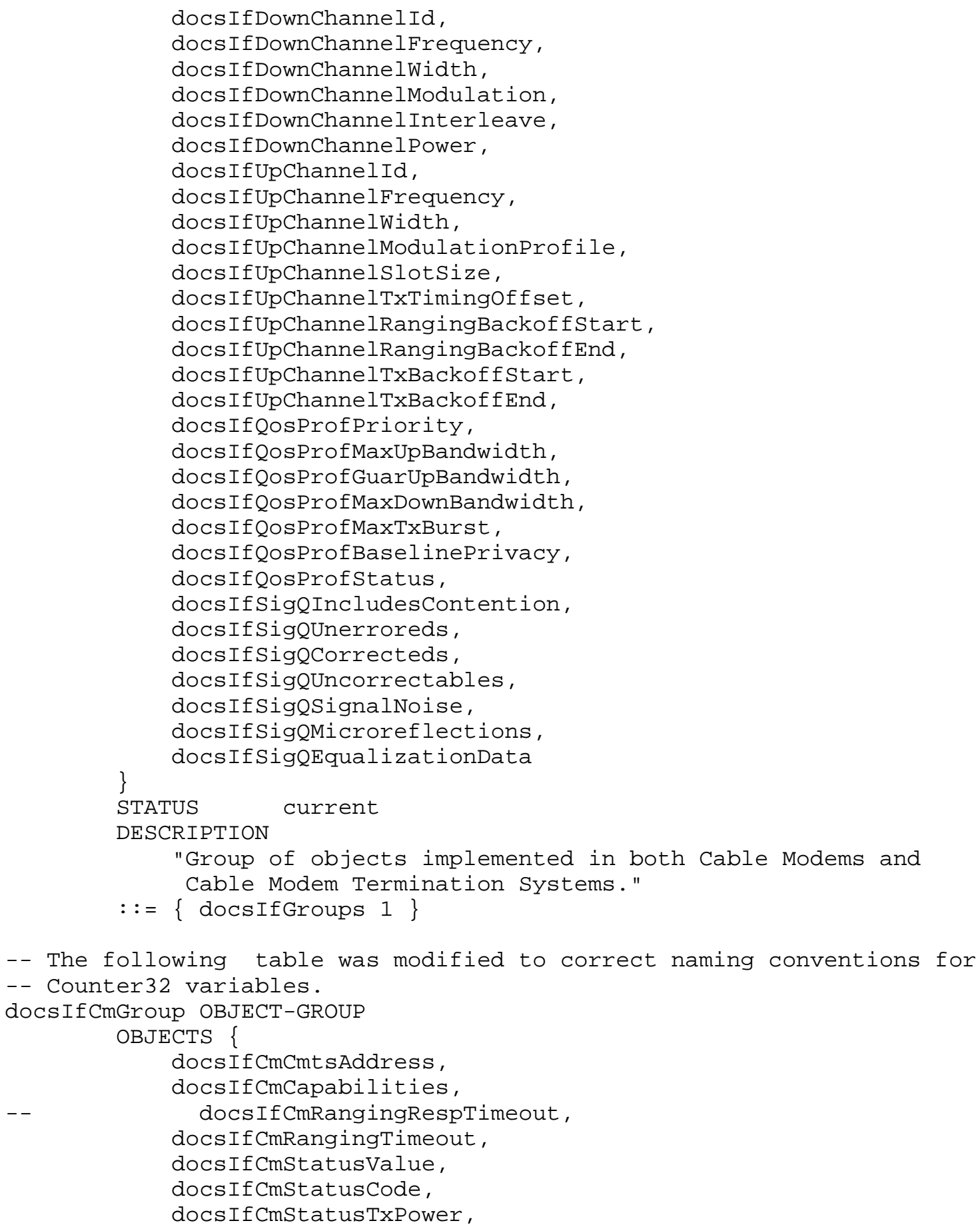

St. Johns

Standard

[Page 66] 


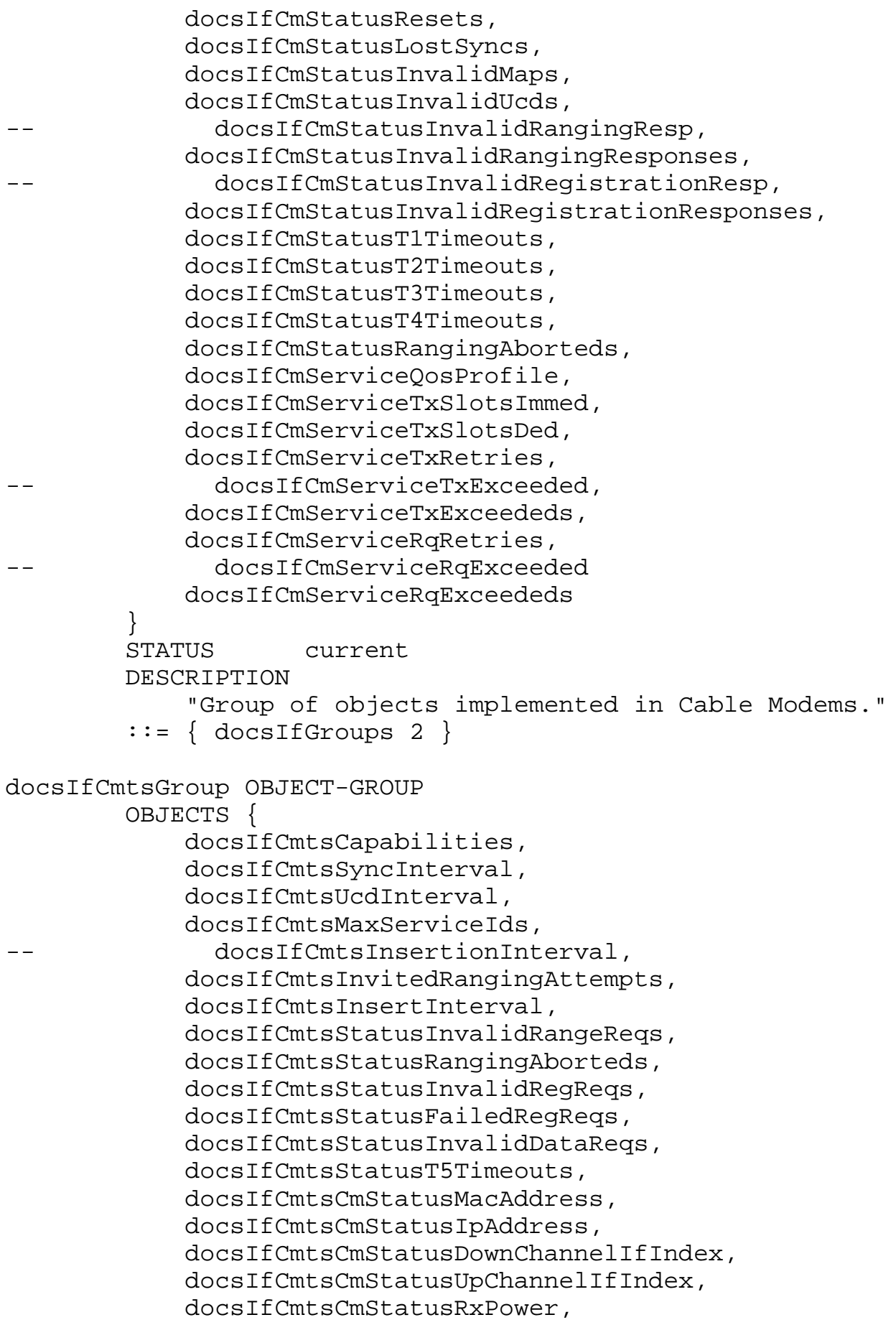

St. Johns

Standard

[Page 67] 


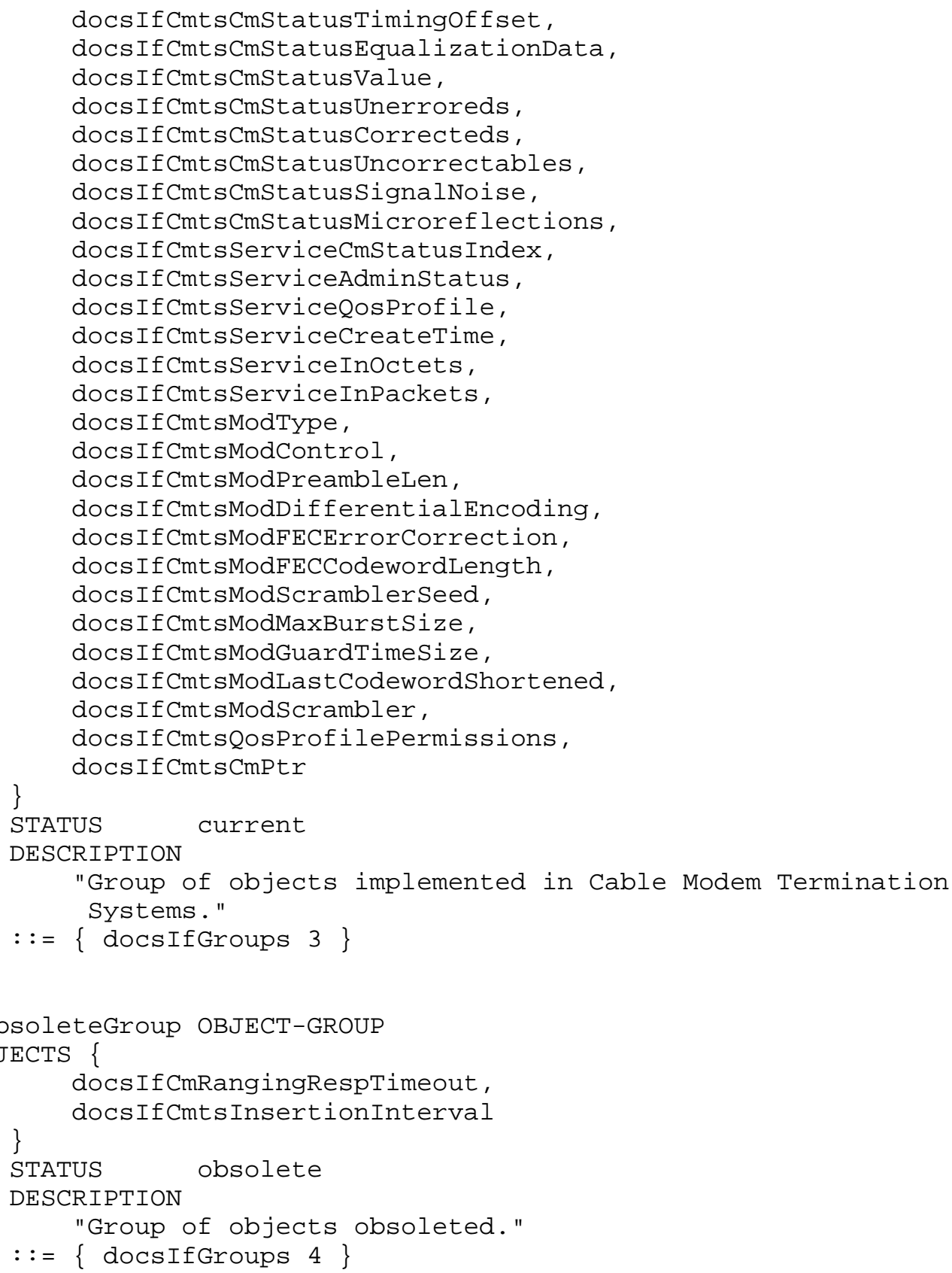

END 


\section{Acknowledgments}

This document was produced by the IPCDN Working Group. It is based on a document written by Pam Anderson from CableLabs, Wilson Sawyer from BayNetworks, and Rich Woundy from Continental Cablevision. The original working group editor, Guenter Roeck of cisco systems, did much of the grunt work of putting the document into its current form. Special thanks is also due to Azlina Palmer, who helped a lot reviewing the document.

6. References

[1] Harrington, D., Presuhn, R. and B. Wijnen, "An Architecture for Describing SNMP Management Frameworks", RFC 2571, April 1999.

[2] Rose, M. and K. McCloghrie, "Structure and Identification of Management Information for TCP/IP-based Internets", STD 16, RFC 1155, May 1990.

[3] Rose, M. and K. McCloghrie, "Concise MIB Definitions", STD 16, RFC 1212, March 1991.

[4] Rose, M., "A Convention for Defining Traps for use with the SNMP", RFC 1215, March 1991.

[5] McCloghrie, K., Perkins, D. and J. Schoenwaelder, "Structure of Management Information for Version 2 (SMIV2)", STD 58, RFC 2578, April 1999.

[6] McCloghrie, K., Perkins, D. and J. Schoenwaelder, "Textual Conventions for SMIv2", STD 58, RFC 2579, April 1999.

[7] McCloghrie, K., Perkins, D. and J. Schoenwaelder, "Conformance Statements for SMIv2", STD 58, RFC 2580, April 1999.

[8] Case, J., Fedor, M., Schoffstall, M. and J. Davin, "Simple Management Protocol", STD 15, RFC 1157, May 1990.

[9] Case, J., McCloghrie, K., Rose, M. and S. Waldbusser, "Introduction to Community-based SNMPv2", RFC 1901, January 1996.

[10] Case, J., McCloghrie, K., Rose, M. and S. Waldbusser, "Transport Mappings for Version 2 of the Simple Network Management Protocol (SNMPv2)", RFC 1906, January 1996. 
[11] Case, J., Harrington D., Presuhn R. and B. Wijnen, "Message Processing and Dispatching for the Simple Network Management Protocol (SNMP)", RFC 2572, April 1999.

[12] Blumenthal, U. and B. Wijnen, "User-based Security Model (USM) for version 3 of the Simple Network Management Protocol (SNMPv3)", RFC 2574, April 1999.

[13] Case, J., McCloghrie, K., Rose, M. and S. Waldbusser, "Protocol Operations for Version 2 of the Simple Network Management Protocol (SNMPv2)", RFC 1905, January 1996.

[14] Levi, D., Meyer, P. and B. Stewart, "SNMP Applications", RFC 2573, April 1999.

[15] Wijnen, B., Presuhn, R. and K. McCloghrie, "View-based Access Control Model (VACM) for the Simple Network Management Protocol (SNMP)", RFC 2575, April 1999.

[16] "Data-Over-Cable Service Interface Specifications: Cable Modem Radio Frequency Interface Specification SP-RFI-I04-980724", DOCSIS, July 1998, http: //www.cablemodem.com/public/pubtechspec/SP-RFI-I04$980724 \cdot \mathrm{pdf}$.

[17] McCloghrie, K. and F. Kastenholz, "The Interfaces Group MIB using SMIv2", RFC 2233, November 1997.

[18] StJohns, M. , "Cable Device Management Information Base for DoCSIS Compliant Cable Modems and Cable Modem Termination Systems", RFC2669, August 1999.

[19] Proakis, John G., "Digital Communications, 3rd Edition", McGraw-Hill, New York, New York, 1995, ISBN 0-07-051726-6

[20] "Transmission Systems for Interactive Cable Television Services, Annex B", J.112, International Telecommunications Union, March 1998 .

7. Security Considerations

This MIB relates to a system which will provide metropolitan public internet access. As such, improper manipulation of the objects represented by this MIB may result in denial of service to a large number of end-users. In addition, manipulation of the docsIfCmServiceQosProfile, docsifCmtsServerQosProfile, and the elements of docsifQosProfileTable may allow an end-user to improve their service response or decrease other subscriber service response. 
This MIB does not affect confidentiality, authentication or authorization of services on a cable modem system. For authentication and authorization, please see the related document "Cable Device Management Information Base for Docsis compliant Cable Modems and Cable Modem Termination Systems" [18]. For confidentiality, the working group expects to issue a MIB which describes the management of the DoCSIS Baseline Privacy mechanism.

8. Intellectual Property

The IETF takes no position regarding the validity or scope of any intellectual property or other rights that might be claimed to pertain to the implementation or use of the technology described in this document or the extent to which any license under such rights might or might not be available; neither does it represent that it has made any effort to identify any such rights. Information on the IETF's procedures with respect to rights in standards-track and standards-related documentation can be found in BCP-11. Copies of claims of rights made available for publication and any assurances of licenses to be made available, or the result of an attempt made to obtain a general license or permission for the use of such proprietary rights by implementors or users of this specification can be obtained from the IETF Secretariat.

The IETF invites any interested party to bring to its attention any copyrights, patents or patent applications, or other proprietary rights which may cover technology that may be required to practice this standard. Please address the information to the IETF Executive Director.

9. Author's Address

Michael StJohns

aHome Network

425 Broadway

Redwood City, CA 94063

Phone: +1 6505695368

EMail: stjohns@corp. home. net 
10. Full Copyright statement

Copyright (C) The Internet Society (1999). All Rights Reserved.

This document and translations of it may be copied and furnished to others, and derivative works that comment on or otherwise explain it or assist in its implementation may be prepared, copied, published and distributed, in whole or in part, without restriction of any kind, provided that the above copyright notice and this paragraph are included on all such copies and derivative works. However, this document itself may not be modified in any way, such as by removing the copyright notice or references to the Internet society or other Internet organizations, except as needed for the purpose of developing Internet standards in which case the procedures for copyrights defined in the Internet Standards process must be followed, or as required to translate it into languages other than English.

The limited permissions granted above are perpetual and will not be revoked by the Internet society or its successors or assigns.

This document and the information contained herein is provided on an "AS IS" basis and THE INTERNET SOCIETY AND THE INTERNET ENGINEERING TASK FORCE DISCLAIMS ALL WARRANTIES, EXPRESS OR IMPLIED, INCLUDING BUT NOT LIMITED TO ANY WARRANTY THAT THE USE OF THE INFORMATION HEREIN WILL NOT INFRINGE ANY RIGHTS OR ANY IMPLIED WARRANTIES OF MERCHANTABILITY OR FITNESS FOR A PARTICULAR PURPOSE.

Acknowledgement

Funding for the RFC Editor function is currently provided by the Internet society. 